INTER NATIONAL MONETARY FUND
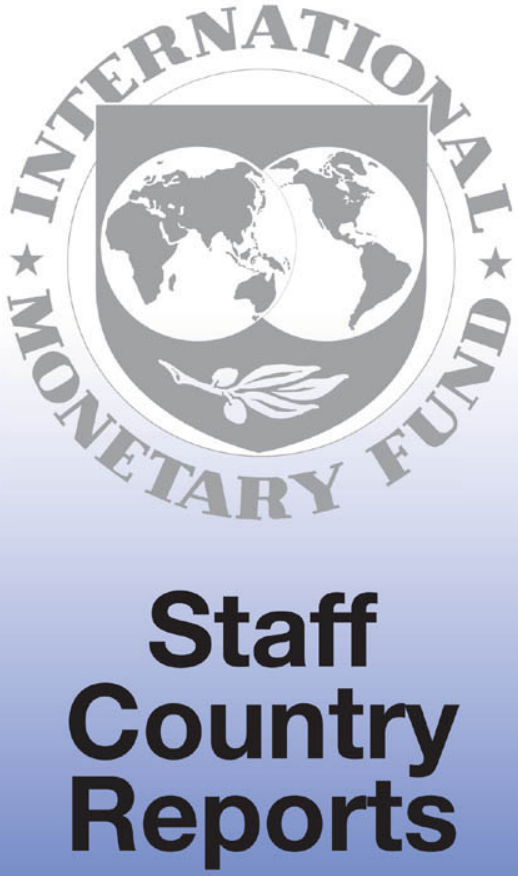


\section{Pakistan: Fourth Review Under the Stand-By Arrangement, Requests for Waivers of Performance Criteria, Modification of Performance Criteria, and Rephasing of Access-Staff Report; Staff Statement and Supplement; Press Release on the Executive Board Discussion; and Statement by the Executive Director for Pakistan.}

In the context of the fourth review under the stand-by arrangement, requests for waivers of performance criteria, modification of performance criteria, and rephasing of access, the following documents have been released and are included in this package:

- $\quad$ The staff report for the Fourth Review Under the Stand-By Arrangement, Requests for Waivers of Performance Criteria, Modification of Performance Criteria, and Rephasing of Access, prepared by a staff team of the IMF, following discussions that ended on April 13, 2010, with the officials of Pakistan on economic developments and policies. Based on information available at the time of these discussions, the staff report was completed on May 3, 2010. The views expressed in the staff report are those of the staff team and do not necessarily reflect the views of the Executive Board of the IMF.

- A staff statement of May 12, 2010 and staff supplement of May 3, 2010 updating information on recent developments.

- $\quad$ A Press Release summarizing the views of the Executive Board as expressed during its May 14, 2010 discussion of the staff report that completed the request and/or review.

- A statement by the Executive Director for Pakistan.

The documents listed below have been or will be separately released.

Letter of Intent sent to the IMF by the authorities of Pakistan*

Memorandum of Economic and Financial Policies by the authorities of

Pakistan*

Technical Memorandum of Understanding*

*Also included in Staff Report

The policy of publication of staff reports and other documents allows for the deletion of market-sensitive information.

Copies of this report are available to the public from

International Monetary Fund $\bullet$ Publication Services

$70019^{\text {th }}$ Street, N.W. • Washington, D.C. 20431

Telephone: (202) 623-7430 • Telefax: (202) 623-7201

E-mail: publications@imf.org Internet: http://www.imf.org

Price: $\$ 18.00$ a copy

\section{International Monetary Fund Washington, D.C.}


INTERNATIONAL MONETARY FUND

PAKISTAN

\title{
Fourth Review Under the Stand-By Arrangement, Requests for Waivers of Performance Criteria, Modification of Performance Criteria, and Rephasing of Access
}

\author{
Prepared by the Middle East and Central Asia Department \\ (In consultation with other departments)
}

Approved by David Owen and Michele Shannon

May 3, 2010

- Discussions were held during February 10-17, 2010 in Dubai. Staff met with Finance Minister Tarin, State Bank of Pakistan (SBP) Governor Raza, Finance Secretary Siddique, and other senior officials. The IMF team consisted of Messrs. Mazarei (Head), Wieczorek, Kock, and Piazza (all MCD), Mr. Jonas (FAD), Mr. Gray (SPR), and Mr. Ross (Resident Representative). Mr. Parvez (Asian Development Bank) participated in the discussions on structural issues. Mr. Tarin resigned effective end-February. His successor, Mr. Abdul Hafeez Shaikh, who was appointed as Finance Advisor to the Prime Minister with the status of finance minister, confirmed program understandings during his visits to Washington in late March and during the Spring Meetings, as well as an April 9-13 staff visit to Islamabad.

- An SBA in the amount of SDR 5.169 billion (about \$7.6 billion, or 500 percent of quota) was approved by the Executive Board on November 24, 2008. On August 7, 2009, the SBA was augmented to SDR 7,235.9 million ( $\$ 11,327$ million, or 700 percent of quota) and extended through end-2010. The third program review was completed on December 23, 2009. Disbursements under the program thus far amount to SDR 4,169.34 million (\$6,414 million), including SDR 713.35 million (\$1,097 million) that was made available for budget financing.

- In the attached Letter of Intent, the authorities request completion of the fourth review under the SBA and the fifth disbursement in the amount of SDR 766.7 million. Out of this amount, SDR 237.75 million will be made available for budget financing.

- Program implementation has been mixed in the context of a difficult political and security environment with delays on the structural agenda and some buildup of quasi-fiscal liabilities, but also progress on important and politically difficult reforms. While performance against endDecember targets was generally in line with program assumptions, preliminary data indicate that the end-March ceilings on the overall budget deficit (excluding grants) and net government borrowing from the SBP were exceeded by 0.4 and 0.2 percent of GDP, respectively. The authorities, therefore, request waivers of nonobservance for the related end-March performance criteria based on the temporary nature of part of this slippage as well as agreement to remedial actions. All other quantitative and continuous performance criteria through end-March were met. The end-December structural benchmark on the submission of the VAT law to parliament was met with a delay (the federal law was submitted on February 25 and provincial laws were submitted to the provincial assemblies in late March). Implementation of an expedited sales tax refund system (end-March structural benchmark) has begun and is expected to be finalized by end-June. The authorities have also committed to remedial steps to address quasi-fiscal issues.

- Looking forward, the authorities have proposed relaxation of the annual budget deficit target by 0.15 percent of GDP (to 5.1 percent of GDP) to accommodate urgent security spending, which will be financed by additional external privatization receipts. At the same time, taking into account Pakistan's improved external position, the end-June 2010 target for the net foreign assets of the central bank will be revised upward by $\$ 300$ million. The authorities request that the related performance criteria for end-June 2010 be modified accordingly. In view of the delay in the fourth review, the authorities also request rephasing of access. Given agreed remedial measures and taking into account the difficult security environment, staff supports the authorities' requests and recommends the completion of the fourth program review. 


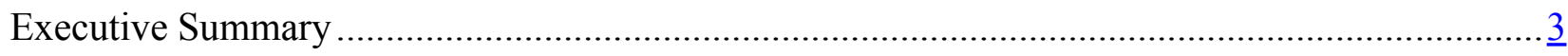

I. Recent Developments and Near-Term Outlook ...................................................

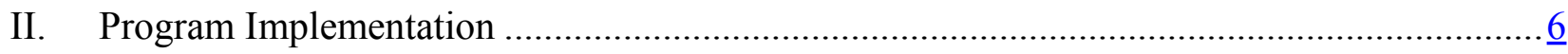

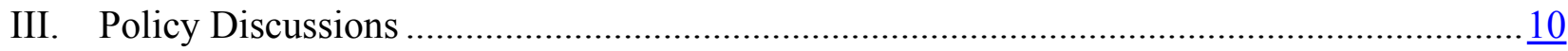

IV. Rephasing and Key Issues for the Fifth Review .................................................... 14

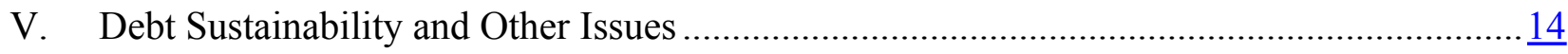

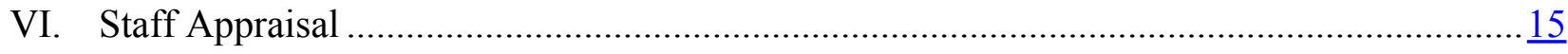

Figures

1. Selected Economic Indicators, March 2009-March 2010 ............................................ 19

2. Financial Market Indicators, November 2008-April 2010 .......................................... 20

3. Real and External Sectors, 2006/07-2010/11 ........................................................ 21

4. Fiscal Policy Indicators, 2007/08-2010/11 ............................................................ 22

Tables

1. Selected Economic Indicators, 2007/08-2009/10 ......................................................... 23

2. Balance of Payments, 2007/08-2009/10 ................................................................. 24

3a. Consolidated Government Budget, 2007/08-2009/10 (in billions of Pakistani rupees) ........ $\underline{25}$

3b. Consolidated Government Budget, 2007/08-2009/10 (in percent of GDP)....................... 26

4. Monetary Survey and Analytical Balance Sheet of the State Bank of Pakistan, 2007/08-2009/10 ........................................................................................... 27

5. Medium-Term Macroeconomic Framework, 2007/08-2014/15 …................................. 28

6. Medium-Term Balance of Payments, 2007/08-2014/15 ................................................... 29

7. Medium-Term Fiscal Framework, 2007/08-2014/15 ................................................ $\underline{30}$

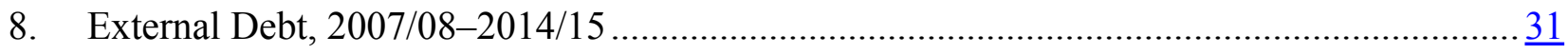

9. Gross Financing Requirements and Sources, 2007/08-2010/11 ................................... $\underline{32}$

10. Indicators of Fund Credit, 2008/09-2015/16 .......................................................... 33

11. Access and Phasing under the Stand-By Arrangement, 2008-10................................... 34

12. Selected Vulnerability Indicators, 2007/08-2014/15 .............................................. $\underline{35}$

13. Financial Soundness Indicators for the Banking System, 2006-09................................. $\underline{36}$

Appendix

Debt Sustainability Analysis

Attachments

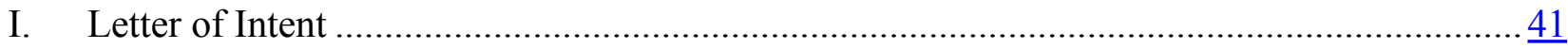

II. Fourth Supplementary Memorandum on Economic and Financial Policies ....................... $\underline{43}$

III. Technical Memorandum of Understanding ............................................................... 54 


\section{EXECUTIVE SUMMARY}

Political and security uncertainties remain high. The military campaign in South Waziristan has continued and resulted in an outflow of population from this and surrounding areas.

Economic conditions are generally improving but inflation has rebounded. Real GDP growth is likely to reach 3 percent in 2009/10, as projected. The projection for headline inflation (y-o-y) for end-June 2010 has been revised upward from 11 to 12 percent. The external current account deficit through end-2009/10 is expected to narrow to below 4 percent of GDP. The exchange rate has been broadly stable in nominal terms, but, due to rising inflation, the real effective exchange rate has appreciated.

While performance at end-December was generally in line with program parameters, the authorities have indicated that the end-March ceilings on budget deficit and net government borrowing from the SBP have been breached. All other quantitative and continuous performance criteria through end-March have been met.

There were some delays in implementing the structural agenda and quasi-fiscal liabilities have risen, but important and politically difficult reforms have now moved forward. The federal VAT bill was submitted to parliament on February 25 and the provincial bills were submitted to the provincial assemblies in late March (prior actions). The implementation of an expedited sales tax refund system (end-March structural benchmark) has begun and is now expected to be completed by end-June. Electricity reform has been slower than expected. And, owing to administrative capacity constraints, the rollout of the scorecard-based targeting system for the Benazir Income Support Program has taken longer than envisaged. As a result, social spending in 2009/10 will likely be below the budgeted target, albeit still higher than in 2008/09.

Discussions focused on the fiscal program for the remainder of 2009/10, the structural reform agenda, and a possible tightening of the monetary policy stance to address the rebound in inflation. Discussions also covered fiscal risks and financial sector issues, including ways to improve financial sector stability and strengthen the regulatory framework. The authorities have also committed to remedial steps to address quasi-fiscal issues.

Security outlays weigh heavily on the near-term fiscal outlook but the resulting fiscal pressures are expected to be largely offset by restraining non-priority current spending. Nevertheless, the authorities believe that the budget deficit target (excluding grants) should be relaxed from 4.9 to 5.1 percent of GDP in order to avoid excessive cuts in development spending. They therefore request that the related end-June performance criterion on the overall budget deficit be modified accordingly. Further, the authorities intend to lock in some of the overperformance on the net foreign assets of the central bank and request to modify the related end-June performance criterion. In view of the delay in the fourth review, the authorities also request the rephasing of access.

Monetary policy will continue to be geared to achieving price stability. To this effect, the SBP changed its monetary policy bias in late March to include the possibility of a tightening.

Staff recommends the completion of the fourth review. Staff supports the requested waivers of nonobservance for the two end-March 2010 quantitative performance criteria on the grounds that their nonobservance was, in part, temporary and that adequate remedial actions have been agreed upon and are expected to be implemented promptly. Staff also supports the requested rephasing of access and modifications to the end-June performance criteria. 


\section{RECENT DEVELOPMENTS AND NEAR-TERM OUTLOOK}

1. Political and security uncertainties remain high. The military campaign in South Waziristan has continued and resulted in an outflow of population from this and surrounding areas. ${ }^{1}$ The political situation has been affected by the Supreme Court ruling of December 17, 2009 rendering the 2007 National Reconciliation Ordinance (NRO) invalid. ${ }^{2}$ Finance Minister Shaukat Tarin resigned effective end-February. His successor, Mr. Abdul Hafeez Shaikh, who was appointed as Finance Advisor to the Prime Minister with the executive powers of finance minister, has reaffirmed the objectives and policy commitments under the program. The rest of the economic team remains in place. In April, the parliament approved the Eighteen Amendment to the Constitution that transfers some key presidential powers to parliament or the prime minister, and enhances provincial autonomy.

2. Economic activity has picked up but inflation has risen more than expected. Real GDP growth is likely to reach 3 percent in 2009/10, as projected. Early indicators point to a drop in wheat output, but the cotton harvest was better than expected. Also, the large-scale manufacturing index increased by 2.5 percent in July 2009-February 2010, relative to the same period in 2008-09. Headline inflation (y-o-y) increased from 10.5 percent in November-December 2009 to 13.7 percent in January 2010 (due mostly to a base effect, but also the unexpectedly large food and energy price increases) and remained elevated at just below 13 percent through March 2010. ${ }^{3}$ Meanwhile core inflation subsided somewhat. As a result, projected headline inflation (y-o-y) for end-June 2010 has been revised upward from 11 to 12 percent, while core inflation is still expected to drop to about 8 percent.

3. The SBP's international reserves have been stable since October. The current account deficit in October 2009-February 2010 was 70 percent lower than in the same period in 2008-09. This improvement reflected mainly import contraction (due to lower commodity prices and a drop in the demand for other imports), lower profit remittances abroad, and stronger inflows of workers' remittances. There was also some rebound in manufacturing exports. The improvement in the external current account has mitigated the impact of lower than expected external financing and helped maintain the official reserves at just above $\$ 11$ billion (about 31/2 months of imports) through end-March. Looking forward, the delayed external financing is expected to be disbursed in the fourth quarter and the external current account through end-2009/10 is expected to narrow to below 4 percent of GDP, enabling the

\footnotetext{
${ }^{1}$ The overall number of internally displaced persons (IDPs) is estimated at 1.25 million at end-November 2009 (compared to a peak level of about 3 million in July 2009). See the report on Pakistani IDPs prepared by the Internal Displacement Monitoring Center (http://www.internal-displacement.org).

2 The NRO was issued by the former President Musharraf on October 5, 2007. It granted amnesty to politicians, political workers, and bureaucrats accused of corruption, embezzlement, money laundering, murder, and terrorism between January 1, 1986, and October 12, 1999. Over 8,000 persons benefited from the NRO, including 34 politicians.

${ }^{3}$ The direct contribution of the energy and transportation component was 2.2 percentage points, reflecting

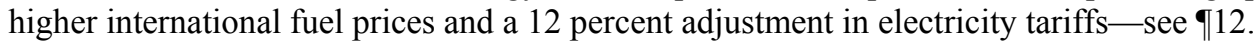


SBP to target a somewhat larger build-up of net foreign assets. This outlook, however, is subject to risks stemming from rising oil prices and a possible decline in remittances.

4. Broad money growth has continued to be weak. There was some pick-up in broad money growth in September-December 2009, which reflected a rebound in bank credit to businesses needing working capital, but money demand weakened in January-March 2010 with both cash and deposits declining in nominal terms. Credit to government continued to grow at double-digit rates (as projected), while credit to private sector remained sluggish. It also appears that the treasury's efforts to replace the shortfall of foreign financing by borrowing from nonbank sources contributed to the weakening of money demand. The SBP continued to manage the difficult liquidity conditions by rolling over its repo position and injecting liquidity via swaps. ${ }^{4}$ After the 50 bps cut in the policy rate in November 2009, the SBP kept the policy rate unchanged at 12.5 percent in its January 30 Monetary Policy Statement, citing the likelihood of an uptick in inflation during the remainder of 2009/10, and maintained this position in its March 27 statement.

\section{Risks to the budget, including on account of quasi-fiscal pressures, have risen.}

These pressures included an increase in the energy sector's "circular debt" and losses from wheat procurement and other commodity operations. Inter-enterprise arrears in the energy sector (circular debt) have increased by about 0.8 percent of annual GDP in the first three quarters of 2009/10 due mainly to nonpayment by energy companies to suppliers and nonpayment of bills by government agencies. ${ }^{5}$ To regularize a major portion of this debt, the government decided to deduct outstanding electricity dues of government institutions from their accounts and refinance the remainder (an estimated 0.5 percent of GDP), together with a portion of the pre-2009/10 circular debt, by issuing additional bonds. Wheat procurement and other commodity operations have added an estimated 0.2 per cent of GDP to quasi-fiscal pressures on account of losses incurred by public procurement agencies. And, the SBP remains concerned about lending for commodity operations crowding out credit to the private sector. ${ }^{6}$ Also, setting high procurement price for wheat has contributed to food price inflation.

6. $\quad$ T-bill placements have proceeded smoothly. The volume targets for T-bill placements were met or exceeded. T-bill rates mirrored the 50 bps cut in the policy rate in November, dropping below 12 percent in early January. T-bill yields have increased, however, following the news of the pick-up in inflation in January and the weighted average T-bill rate stood at about 12.3 percent in mid-April. Meanwhile, the secondary market for

\footnotetext{
${ }^{4}$ The SBP increased its FX swap position by $\$ 320$ million in the last quarter of 2009 and a further $\$ 85$ million in the first quarter of 2010.

${ }^{5}$ An audit of the pre-2009/10 overdue liabilities of the public sector energy companies confirmed that the debt to be assumed by the newly created holding company (in May 2010) amounts to Rs 216 billion (1.4 percent of GDP). Meanwhile, the government has assumed the interest payments on this debt.

${ }^{6}$ The outstanding stock of commodity credits at commercial banks' balance sheets at end-March 2010 was double the end-March 2009 level and amounted to nearly 2 percent of GDP.
} 
treasury bills and other government paper has deepened with the SBP's establishment of a new electronic bond-trading platform in January.

7. The exchange rate has been broadly stable in nominal terms. The rupee depreciated from Rs 83 per U.S. dollar at end-September 2009 to Rs 84-85 per U.S. dollar in FebruaryApril 2010. However, due to rising inflation, the real effective exchange rate appreciated by 1.6 percent in the last three months of 2009. The cessation of the provision of foreign exchange for oil imports by the SBP in mid-December did not have a significant impact on the interbank foreign exchange market.

8. The stock market has continued its upward trend. There was a downward correction of about 10 percent in late 2009, which reflected increased political and security uncertainties in the wake of the campaign in South Waziristan, and the downgrading of the ratings of several Pakistani banks by Merrill Lynch. But the market recovered subsequently, following the announcement of better than expected earnings by several large banks and companies. Analysts also noted a positive impact owing to the completion of the third program review. Sovereign debt ratings of Pakistan have not changed since November, while Pakistan's EMBIG spread dropped from 700 bps in November 2009 to 460 bps in mid-April.

9. Poverty has likely risen. Pakistan saw a significant decline in poverty during the precrisis period with the share of the population living in poverty dropping from 34.5 percent in 2001/02 to 17.2 percent in 2007/08. However, the economic slowdown and the erosion of purchasing power caused by inflation suggest that these gains in poverty reduction may have been partly reversed. The 2007/08 household survey results indicated that poverty started rising already toward the end of that fiscal year. While the continuation of this trend is yet to be confirmed by the next household survey, it is to be noted that food and non-food prices rose by 23.7 and 18.4 percent, respectively, between $2007 / 08$ and 2008/09, resulting in a 21 percent reduction in purchasing power.

\section{PRogRAM IMPLEMENTATION}

\section{Except for a small overrun on the budget deficit target, all performance criteria} through end-December were met. Revenue collection by the Federal Bureau of Revenue (FBR) in July-December fell short of the program's target by close to 0.2 percent of GDP and there was a similar shortfall in provincial tax and nontax revenue, but the authorities offset revenue shortfalls by restraining current and development spending, while accommodating 0.3 percent of GDP in additional security spending. As a result, the budget deficit (excluding grants) exceeded the program target by 0.02 percent of GDP. ${ }^{7}$

\footnotetext{
${ }^{7}$ Current spending was lower due mainly to a postponement in the payout of subsidies $(0.4$ percent of GDP in, notably, electricity tariff differential and wheat subsidies). There were also some savings in interest payments and unidentified (mainly provincial) spending.
} 
11. Two quantitative performance criteria for end-March were missed. Preliminary information indicates that the performance criteria on the SBP's net foreign assets (NFA) and domestic assets were met by wide margins, but the ceilings on the overall budget deficit (excluding grants) and net government borrowing from the SBP were missed.

- The budget deficit target was exceeded by an estimated 0.4 percent of GDP (including 0.2 percent of GDP based on application of program adjustors), due mainly to the revenue shortfall, which reached an estimated 0.7 percent of GDP in the first three quarters of 2009/10. While the authorities made significant cuts in current and, to a smaller extent, development spending to reduce the slippage, these savings were partially absorbed by an estimated 0.4 percent of GDP in additional security outlays in the third quarter, which included spending on the reconstruction of infrastructure in the areas affected by the conflict. ${ }^{8}$ At the same time, shortfalls in grants for internally displaced persons (IDP grants) and Tokyo-related multilateral support increased the margin of nonobservance via adjustors.

Pakistan: Fiscal Outcome, July 2009-March 2010 and FY 2009/10 Program

(In percent of GDP)

\begin{tabular}{|c|c|c|c|c|c|c|}
\hline & \multicolumn{2}{|c|}{ July-December 2009} & \multicolumn{2}{|c|}{ July 2009-March 2010} & \multicolumn{2}{|c|}{ FY 2009/10 } \\
\hline & $3^{\text {rd }}$ Review & Actual & $3^{\text {rd }}$ Review & Preliminary & $3^{\text {rd }}$ Review & $4^{\text {th }}$ Review 2/ \\
\hline Revenue & 6.5 & 6.0 & 10.2 & 9.5 & 14.6 & 14.4 \\
\hline Tax revenue & 4.7 & 4.5 & 7.4 & 6.9 & 10.7 & 10.4 \\
\hline Nontax revenue & 1.8 & 1.6 & 2.9 & 2.6 & 3.9 & 4.0 \\
\hline Expenditure 1/ & 9.1 & 8.7 & 14.3 & 13.7 & 19.6 & 19.5 \\
\hline Current & 7.6 & 7.4 & 11.8 & 11.3 & 16.0 & 16.2 \\
\hline Of which: Social safety net & $\ldots$ & 0.1 & $\ldots$ & 0.2 & 0.6 & 0.4 \\
\hline Development & 1.4 & 1.3 & 2.5 & 2.4 & 3.4 & 3.4 \\
\hline Balance excluding grants & -2.7 & -2.7 & -4.0 & -4.2 & -4.9 & -5.1 \\
\hline \multicolumn{7}{|l|}{ Memorandum items } \\
\hline Additional security spending $3 /$ & $\ldots$ & 0.3 & $\ldots$ & 0.7 & 0.4 & 1.0 \\
\hline Of which: Wage increase & $\ldots$ & $\ldots$ & $\ldots$ & 0.1 & 0.2 & 0.2 \\
\hline
\end{tabular}

$1 /$ Includes statistical discrepancy.

$2 /$ The difference in the revenue-to-GDP ratio between the $3^{\text {rd }}$ and $4^{\text {th }}$ reviews is 0.1 percent of GDP, as discussed in $\llbracket 14$.

The difference shown in this table appears larger due to rounding.

$3 /$ Relative to the $2009 / 10$ budget.

- The target for net government borrowing from the central bank was exceeded by 0.2 percent of GDP. The authorities had expected significant external financing inflows in the last weeks of March to settle the intra-quarter overdraft. These inflows are now expected in the fourth quarter of 2009/10. Meanwhile, the authorities will strengthen

\footnotetext{
${ }^{8}$ Factors that led to the nonobservance of the end-March budget deficit target were discussed during the February meetings with the authorities in Dubai and policy understandings that were reached are reflected in the agreement on the revised end-June budget deficit target (see $\$ 14$ ) and external financing assumptions (see $\$ 15$ ).
} 
government liquidity management and reduce recourse to the SBP overdraft to ensure that targets for borrowing from the SBP are met.

\section{There were some delays in implementing the structural agenda, but important and politically risky reforms have moved forward.}

- The federal $\boldsymbol{V A T}$ bill was submitted to the parliament on February 25. The provincial VAT bills were submitted to the provincial assemblies in late March, following consultations with provinces and preliminary parliamentary discussions on the federal law. ${ }^{9}$ With the submission of the federal VAT bill and four mutually consistent provincial VAT bills, the authorities met the prior actions for the fourth program review. However, the Sindh government also submitted a parallel Sales Tax on Services Bill, which poses a risk to the timely passage of the VAT bill and the overall consistency of the VAT package. The authorities indicated that the differences with Sindh would be resolved soon. They also recognize that the federal and provincial components of the VAT legislative package will need to be fully consistent to avoid problems of cascading and tax competition. Also, the government's proposals seek to establish a uniform tax rate for most goods and services and to limit the scope of zero-rating and exemptions in order to ensure that the VAT yields the required revenue increase (SMEFP q11).

- There has been some progress on tax administration reform. The implementation of the expeditious refund system (ERS) in all Regional Taxpayers Offices (RTOs) and Large Taxpayers Units (LTUs) (end-March structural benchmark) has begun and is expected to be completed by end-June. The ERS enables timely and accurate verification of sales tax refund claims submitted by exporters and mitigates the risk of refunding fraudulent claims. The ERS was tested at the Islamabad LTU on a pilot basis. Now the pilot has been extended to the Lahore RTO and the system is expected to be fully deployed by end-June in all RTOs and LTUs. To this effect, the FBR issued a regulatory order in late March requesting manufacturing exporters registered at the Lahore RTO to start submitting their refund claims electronically through the ERS from the April sales tax period onwards. Manufacturing exporters registered at all other RTOs and LTUs will begin to use the ERS in the July tax period (SMEFP $\mid 12$ ).

- Electricity tariff adjustments have been slower than expected. Full implementation of the 6 percent adjustment agreed with the World Bank and the Asian Development Bank (ADB) for October was completed in December. Subsequently, tariffs were raised by 12 percent on January 1 , as scheduled. ${ }^{10}$ A further 6 percent increase was originally expected on April 1; the authorities have now indicated that they will implement this

\footnotetext{
${ }^{9}$ The decision to have separate but closely coordinated VAT legislation at the two levels reflected the need to align the proposed laws with constitutional provisions allocating powers to levy general sales taxes on goods and services exclusively to federal and provincial governments respectively.

${ }^{10}$ Electricity tariffs were raised by about 60 percent during September 2008-January 2010.
} 
increase later, back-dated to April 1, although no specific timeline has been set. Monthly adjustments on account of fuel cost changes have continued, albeit with some lags; however, the quarterly determination of the required tariff adjustment for July-September was not implemented, but covered by subsidies; as of end-March, the authorities had transferred to the Pakistan Electric Power Company the Rs 55 billion (0.4 percent of GDP) budgeted for tariff differential subsidies in 2009/10 and an additional Rs 12 billion (0.1 percent of GDP) in budgeted funds to the Karachi Electric Supply Company. While the authorities had previously indicated that the quarterly adjustment (for OctoberDecember, 2009) would be implemented in early April and the quarterly adjustment (covering January-March, 2010) would be notified and implemented on time, they have later questioned the feasibility of these (as well as further monthly fuel price) adjustments. The authorities continue discussions with the World Bank and the ADB staffs on a way forward on these issues, including on actions to eliminate tariff differential subsidies, and have committed to establishing by end-June 2010 a comprehensive framework (agreed with the World Bank and the ADB) for dealing with cost pressures and supply shortages in the electricity sector (SMEFP $\llbracket 14$ ).

- Owing to administrative capacity constraints, the rollout of the scorecard-based targeting system for the Benazir Income Support Program (BISP) has taken longer than envisaged. As a result, social spending in 2009/10, though still significantly higher than in 2008/09, will likely be below the budgeted amount. Also, 15-20 percent of BISP payouts will be delivered to beneficiaries identified before the scorecard was introduced. The authorities (with help from World Bank staff) are exploring ways to accelerate the

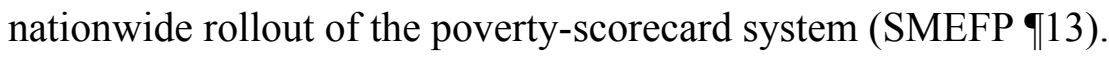

- The amendments to the Banking Companies Ordinance (BCO) were approved by the National Assembly on February 8 and are expected to be approved by the Senate in early May. These changes will strengthen the SBP's ability to deal with problem banks (SMEFP -26). The amendments to the SBP Act, aiming at enhancing operational independence of the central bank, were introduced to the National Assembly on March 17 and will be considered by the Committee on Finance and Revenue in the coming weeks.

- The draft Corporate Rehabilitation Act (CRA) has been revised to create a mechanism that could allow the write-down, write-off, or subordination of creditor claims for a small group of the largest distressed companies in the economy. World Bank staff has raised concerns about several aspects of the revised draft, and is recommending that it be realigned with the best practices in bankruptcy law before it is submitted to parliament. World Bank experts have pointed out that, with these new provisions, the operation of the CRA could raise issues of financial stability (because banks would be required to absorb the loss of significant levels of non-performing loans), and concerns about fiscal discipline (because of the contemplated use of public funds to purchase claims and assets with no clear market value). The authorities are reviewing the draft to address these shortcomings. 


\section{Policy Discussions}

\section{Policy discussions focused on the fiscal program for the remainder of 2009/10, the} structural reform agenda, and a possible tightening of the monetary policy stance to address the rebound in inflation. Staff also discussed with the authorities measures to address fiscal risks and financial sector issues, focusing on ways to improve financial sector stability and strengthen the regulatory framework.

\section{Fiscal policy}

\section{Security weighs heavily on the near-term fiscal outlook, and, while identifying} measures to address most of the end-March slippage, the authorities saw the need to relax the 2009/10 budget deficit target by 0.15 percent of GDP to 5.1 percent of GDP.

- The authorities believe that the end-June budget deficit target (excluding grants) should be relaxed from 4.9 percent of GDP to 5.1 percent in order to allow room for additional security spending while avoiding excessive cuts in development spending and protecting priority social spending. They consider the revised budget deficit target achievable under ambitious but realistic revenue assumptions and further expenditure restraint.

- Revenue through end-June is projected to fall short of the program target agreed at the time of the third program review by 0.1 percent of GDP. ${ }^{11}$ This projection takes into account: (i) the performance in the first three-quarters of 2009/10; (ii) revenue collection measures which are expected to boost revenue in the last quarter by 0.2 percent of GDP; and (iii) 0.6 percent of GDP in nontax revenue from the U.S. Coalition Support Fund $(\mathrm{CSF})^{12}$ expected in the fourth quarter (see below).

- Current expenditure is projected to be higher than envisaged, due primarily to additional security spending (over 0.5 percent of GDP in the second half, mainly in the third quarter). As noted above, most of the additional security spending have been accommodated by restraining other current spending, but the authorities have indicated that the overall amount of current outlays will need to increase by $0.1-0.2$ percent of

\footnotetext{
11 The FBR continues to aim at the 2009/10 collection target of Rs 1,380 billion. This includes Rs 44 billion ( 0.3 percent of GDP) from the execution of specific court judgments on past due taxes and targeted actions against tax avoidance and tax evasion, including audits to deal with non-filers and underreporting taxpayers (SMEFP 112). Of this amount, Rs 10 billion was recovered in the third quarter of FY 2009/10 and the remainder is expected to be recovered by end-June. Nontax revenue of about Rs 20 billion or 0.15 percent of GDP arise from an exceptional transfer of retained profits from the SBP reserve fund to compensate for the shortfall in revenue. Staff cautioned against such ad hoc transfers.

12 The CSF was established in 2001 to support U.S. allies for some of the costs incurred in the fight against extremist violence. Pakistan has been the primary beneficiary of the CSF.
} 
GDP to avoid undue cuts in priority spending. They agreed however that any additional subsidies (e.g., to cover losses from wheat procurement— see 917 ) would need to be accommodated within the overall unchanged envelope for subsidies.

- Development spending is currently projected to remain as envisaged at the time of the third program review, except for minor cuts in federal development outlays (less than 0.1 percent of GDP). The authorities indicated, however, that any additional fiscal pressures, including from revenue underperformance, would be offset by further restraint in non-priority federal development spending and, to this effect, identified appropriate contingencies of 0.3 percent of GDP.

- Attaining the revised fiscal deficit target will depend on obtaining the budgeted receipts (refunds) from the CSF. The CSF receipts projected for 2009/10 amount to $\$ 1.35$ billion (nearly 0.8 percent of GDP). Of this amount, Pakistan has received thus far $\$ 349$ million ( 0.2 percent of GDP). The Pakistani and U.S. authorities are working toward a timely release of the remaining funds.

15. The feasibility of the revised fiscal deficit target also hinges on higher privatization receipts (from abroad), which are expected to cover the additional budget financing needs and shortfalls in overall external grants. The external financing assumptions and program adjustors have been changed to reflect the offsetting effects of reduced projections for IDP grants and multilateral financing, and a substantial increase in privatization receipts and non-IDP grants. ${ }^{13}$ As a result, the relaxation of the fiscal deficit target will not entail an increase in the government's domestic financing needs and will not affect the public debt-toGDP ratio. Moreover, the authorities agreed to lock in a part of the over-performance relative to the end-December NFA target to ensure that the relaxed fiscal position does not compound external vulnerabilities; to this effect, the NFA target for end-June 2010 has been revised upward by $\$ 300$ million. Fund financing to the budget at year-end is expected to be about $\$ 700$ million; about a half of the IMF disbursements to the budget will be reimbursed to the SBP in the last quarter of 2009/10, as envisaged at the time of the third program review.

\section{Preparations for the medium-term budget framework for 2010/11-12/13 have} started. The initial Budget Strategy Paper has been approved by the cabinet. The mediumterm strategy (which will lay the basis for the 2010/11 budget) aims at reducing the fiscal

\footnotetext{
${ }^{13}$ In the Technical Memorandum of Understanding, reference baselines and program adjustors have been changed to reflect the revised external financing assumptions. As such, the projected shortfall (of nearly 0.3 percent of GDP) from the originally programmed level of IDP grants will not imply adjustment in the overall deficit target under the revised program framework. The projection for external privatization receipts has been revised upward by 0.3 percent of GDP (from $\$ 266$ million to $\$ 800$ million). This comprises proceeds from the sale of Pakistan Telecommunication Company Ltd, which had been withheld by the investor (Etisalat) since 2006 from the total amount of $\$ 2.6$ billion pending the transfer of related properties. In addition, all adjustors to the budget deficit target, including on account of the shortfall in privatization receipts, have been made asymmetric to cap the fiscal deficit target at 5.1 percent of GDP.
} 
deficit (excluding grants) by about $2 \frac{1}{2}$ percent of GDP over three years while increasing the share of development spending (linked to foreign grants). The strategy also seeks to achieve a significant devolution of resources from the federal budget to the provinces (from 4.4 percent of GDP in 2009/10 to 7.4 percent by 2012/13), consistent with understandings reached in the context of the National Finance Commission (NFC) Award last December. ${ }^{14}$ To this effect, the divisible pool of resources is projected to increase by 3 percent of GDP, of which 2.4 percent will be on account of the introduction of the VAT.

\section{The authorities continue to seek ways to address fiscal risks in the public enterprise} sector. They plan to curb the losses of the public enterprises, estimated at Rs 200-250 billion $\left(1 \frac{1}{3}-12 / 3\right.$ percent of GDP) on an annual basis, by restructuring eight major entities (SMEFP $\mid 21$ ). ${ }^{15}$ To improve transparency, the Ministry of Finance has also started to publish on its website information on the stock of government guarantees. ${ }^{16}$ The recurrent problem of circular debt in the energy sector is to be resolved by strengthening the management of the regional electricity distribution companies (their top managers, including CEOs, will be subject to performance contracts to reduce companies' losses and improve their financial management), while the payment arrears to the electricity companies incurred by government agencies will be cleared by making at-source deductions from their budget allocations (SMEFP 115). Regarding wheat procurement and other commodity operations, the authorities aim to cover the losses of the government procurement agencies within the existing budget envelope for subsidies. The wheat procurement target for the 2010 season has been reduced and the overall amount of commodity credits will be subject to a cap (SMEFP \16).

18. The authorities reconfirmed the timetable for VAT implementation. In parallel to pressing forward with the legislative agenda (with passage of the VAT laws now expected by end-May 2010), the authorities are making progress on technical preparation for the implementation of the VAT by July 1, 2010. The key steps remaining include procuring the information technology and supporting equipment (including to manage refunds), stepping up staff training at the FBR, and an ongoing information campaign to familiarize taxpayers with the VAT framework.

\footnotetext{
14 The NFC Award determines the distribution of revenue between the federation and the provinces for 2010/11-2014/15. Certain taxes are pooled, and then redistributed according to the agreed NFC formula. The NFC Award agreed upon in December 2009 increased the share of Baluchistan (the poorest province).

${ }^{15}$ Pakistan Railways, Pakistan Electric Power Company (PEPCO), Pakistan International Airlines (PIA), Pakistan Steel, National Highway Authority (NHA), Utility Stores Corporation (USC), Trading Corporation of Pakistan (TCP), and Pakistan Agricultural Storage and Services Corporation (PASSCO). Recently, the government decided to replace the incumbent CEOs and Boards of these eight companies.

${ }^{16}$ The stock of guarantees outstanding at end-March was Rs 566.6 billion (3.7 percent of GDP).
} 


\section{Monetary and exchange rate policies}

\section{Monetary policy will continue to focus on combating inflation and strengthening}

international reserves. Headline inflation (y-o-y) is expected to subside in the months ahead, but there is a risk that inflationary expectations become more entrenched. In this context, the SBP indicated that it would change the monetary policy bias toward tightening (and later signaled it in its March 27 decision) and, subsequently, it would increase the policy rate if inflation continues to exceed expectations. The SBP will keep the exchange rate flexible, which will help ensure that the accumulation of international reserves continues.

\section{The SBP will also seek ways to broaden the government securities market and} increase the space for private sector credit. In this regard, a reduction in credit related to commodity operations and the clearance of public enterprise debt (e.g., in the energy sector) will help. The SBP considers that additional space may come from the development of the debt securities market, including with the use of the electronic bond-trading platform, which is expected to attract more nonbank investors to the government securities market.

\section{Financial sector}

21. Financial soundness indicators through end-December point to a continued, albeit slow, deterioration in bank conditions. Nonperforming loans (NPLs) increased further in the last quarter of 2009, and regulatory measures adopted last fall to foster the restructuring of NPLs have not yet yielded the intended results. NPLs are generally adequately provisioned for and banks remain profitable and generally well-capitalized, but a few small and one medium-sized bank face capital deficiencies, partly related to increases in minimum capital requirements and capital adequacy ratios. Most of these banks have agreed with the SBP on plans to comply with the increased requirements by mid-2010, including through mergers and fresh capital injections by foreign and domestic partners. The exposure of Pakistani banks to Dubai World is reported to be limited to one bank that has adequate capital to absorb the possible losses. On a separate matter, there are concerns about the "fit and proper" qualifications of some bank managers and owners, as well as a generalized concern over governance (e.g., related to directed lending) in some public banks. The SBP intends to draw on its strengthened supervisory powers (arising from BCO amendments) to address these concerns.

\section{The SBP is evaluating the modifications proposed by the Basel Committee to} strengthen the regulatory framework. In many respects, the framework used in Pakistan already meets the proposed enhanced requirements, but the SBP will conduct a full impact assessment of the Basel Committee proposals in the near future. A preliminary assessment suggests that the introduction of the additional criteria would confirm that Pakistani banks are liquid and well-capitalized. Banks' capital is largely composed of Tier 1 capital, a large share of banks' assets is held in liquid (government) securities, and banks' exposure to derivative products is very low. 


\section{REPHASING AND KEY ISSUES FOR THE FIFTH REVIEW}

23. Given the proposed rephasing of access and the resulting change in the number and schedule of reviews, the next (fifth) review will focus on the implementation of the $V A T$ and reaching agreement on the 2010/11 budget. In view of the delay in the fourth review, the authorities have requested rephasing of access under the arrangement. Specifically, in order to allow a sufficient period prior to the next review to advance core elements of the reform agenda, the authorities have requested that the last three purchases (and associated reviews) under the arrangement be consolidated into two, with access of about SDR 1.15 billion (equivalent to about 111 percent of quota) being made available at the completion of each review. Accordingly, the fifth review, originally scheduled for June, could now take place in August. It will assess the performance of Pakistan under the quantitative performance criteria through end-June 2010 and will focus on the structural agenda that was originally to be reviewed both under the fifth and the sixth reviews:

- Implementation of the VAT (structural benchmark for July 1, 2010). The remaining intermediate steps include: (i) the approval (by end-May) of the federal VAT bill by parliament and consistent provincial VAT bills by the provincial assemblies; (ii) promulgation of the regulations ensuring their orderly implementation within a month of their approval; and (iii) legislation to harmonize the existing tax laws with the VAT Act (expected to be submitted in early June, 2010).

- Reaching agreement on the 2010/11 budget. ${ }^{17}$ The next year's budget must be consistent with the principles of macroeconomic stability and public debt sustainability, taking into account the accrual of liabilities stemming from quasi-fiscal operations. Availability of external financing is likely to be a key constraining factor. The agreement on the 2010/11 budget and the supporting macroframework is also needed to establish the quantitative performance criteria for end-September 2010, which will constitute conditionality for the sixth review and seventh (final) purchase under the SBA.

\section{DebT SustainabiLity AND Other ISSUES}

24. Debt sustainability outlook has remained broadly unchanged relative to the third program review. The updated debt sustainability analysis (DSA) shows that external debt remains low under the medium-term baseline scenario, while the public debt remains high but is expected to decline gradually.

- External debt edges up from 31 percent of GDP in 2009/10 to 331/2 percent in 2010/11$11 / 12$, but declines steadily thereafter. It would rise to 40 percent in the event of shocks to oil prices or FDI, but exceed 50 percent only in the event of a simultaneous shock to

\footnotetext{
${ }^{17}$ The budget is expected to be submitted to parliament in early June.
} 
growth, the current account, and FDI combined with a real depreciation. Thus the risks to external solvency are not large, but need to be contained.

- Public debt, including Fund credit to the budget, is projected to peak at $561 / 2$ percent of GDP in 2009/10 (and 61 1/2 percent of GDP in 20010/11 including all liabilities to the IMF), and then decline to below 50 percent by $2014 / 15$. Simulations show that growth, primary balance and interest rate shocks, as well as a combined shock involving all these components, would not push the public debt-to-GDP ratio above 60 percent. However, the 30 percent depreciation shock would push it above 70 percent, while the contingent liability shock would raise it to 65 percent. Also, Pakistan's public debt-to-revenue ratio exceeds 400 percent (more than twice the emerging markets countries' average) and interest payments consume about one third of budget revenues (more than in any emerging market country). Public debt is, therefore, a source of vulnerability and needs to be reduced. The authorities recognize this need and in the preliminary version of the Budget Strategy Paper project to bring public debt below 50 percent of GDP in the medium term.

\section{In January the SBP removed the exchange restriction on advance payments} against letters of credit. In doing so, it reiterated the need for banks to assess the bona fide nature of the underlying transactions.

26. An update of the March 2009 safeguards assessment of the SBP was completed in February 2010. It found that efforts had continued to strengthen the SBP's safeguards framework. The SBP also accepted the proposed timetable for implementing most safeguards measures and agreed that the external auditors should proceed with confirming the level of foreign reserves at end-2009 directly with their counterparties. Amendments to the SBP Act are pending parliamentary discussion and approval.

\section{StafF APPRAISAL}

27. The authorities have committed to addressing the third quarter fiscal slippage and continue to press ahead with structural reforms in the context of a difficult political and security environment. Looking forward, achieving fiscal objectives will remain the key policy challenge, particularly given that the security situation is affecting both revenue and expenditures. At the same time, the recent pick-up in inflation re-emphasizes the importance of fiscal prudence.

28. Staff supports the relaxation of the 2009/10 budget deficit target (excluding grants) by 0.15 percent of GDP to 5.1 percent of GDP. The additional fiscal space will allow for additional security spending, and financing it through privatization receipts would avoid further debt creation. However, achieving the revised fiscal deficit target will require strong tax collection efforts and expenditure restraint. In particular, the FBR revenue target is ambitious; attaining it will require political backing and decisive steps to address tax evasion 
and avoidance. Moreover, further ad-hoc transfers from the SBP to cover revenue shortfalls should be avoided and the government's liquidity management should be strengthened (e.g., through pre-financing) to limit recourse to the SBP overdraft. Expenditure restraint will be critical, but expenditure compression should be limited to non-priority projects while protecting spending on poverty reduction and assistance to the internally displaced persons. The revised budget deficit target is also subject to risks related to the timing of the external inflows and unforeseeable security-related spending pressures.

29. Despite institutional and political hurdles, the authorities have made progress (albeit with some delays) in preparing for the introduction of the VAT. Looking forward, further efforts are needed to ensure that the VAT is implemented on July 1, as scheduled. The federal and the provincial parts of the VAT legislative package need to be made consistent to avoid problems of cascading and tax competition. Moreover, it will be critical to limit exemptions and properly calibrate the VAT rate in order to ensure an adequate revenue yield. The introduction of a broad-based VAT is crucial for boosting tax revenue in the medium term. The pool of budgetary resources, an increasing share of which will be devolved to the provinces, must increase significantly to support the new revenue-sharing arrangements and allow for high priority spending. The transfer of revenues to provinces must be accompanied by a transfer of spending responsibilities, which will require a significant strengthening of budget execution functions at the provincial levels.

\section{The rollout of the enhanced targeting mechanism under the BISP needs to be} accelerated. In this regard, strengthening of the social safety net is of utmost importance to address the likely deterioration in poverty indicators.

31. Risks to the public finance from quasi-fiscal operations need to be contained. Plans to curb quasi-fiscal losses are welcome, and need to be implemented. In this context, the initiative to restructure the eight large SOEs needs to be translated into action. In the electricity sector, a comprehensive framework to eliminate tariff differential subsidies is urgently needed. Losses from wheat procurement should be dealt with in a transparent manner and credit for commodity operations should be contained to allow adequate space for private credit. Overall, the authorities will need to ensure that spending on subsidies remain within the budgeted level.

32. Monetary policy should be geared at restoring the trend toward price stability. Inflation must not become entrenched at current levels, as it will increase poverty, impede economic recovery, and harm long-term growth prospects. The SBP should raise the policy rate promptly if inflationary pressures do not abate as expected. SBP's efforts need to be supported by fiscal policy. 
33. The SBP should be empowered to better support financial sector stability. Key areas for improvement include strengthening governance in public financial institutions, strictly enforcing "fit and proper" rules, and fostering bank compliance with capital adequacy requirements. In this regard, the timely approval of the amendments to the Banking Companies Ordinance by the Senate is of utmost importance. Similarly, it would be important to obtain early parliamentary approval of the amendments to the SBP Act.

34. External financing remains a risk to the program. A strengthened current account will allow for a continued accumulation of reserves; Pakistan's capacity to repay the Fund remains broadly adequate and will not be affected by the requested rephasing of access. However, the balance of payments continues to be subject to uncertainties including the timing of donor financing and privatization receipts.

35. Compared to a year ago, the economy is clearly in a much less risky state, but vulnerabilities remain considerable. Despite pressures on the security front and a difficult domestic political setting, the authorities have made progress in advancing a number of politically difficult reforms, including raising electricity and fuel prices and tightening monetary policy. These steps have helped stabilize the economy. Looking ahead, the budget will remain subject to spending pressures on security and financing uncertainties, and monetary policy will need to contend with continued inflationary risks. Moreover, a number of tough structural reforms need to be made, especially the introduction of VAT. And, with a weak economy and high inflation, poverty is rising. These challenges call for strong policies.

36. Staff supports the requested waivers and program modifications. Staff supports the requested waivers for the nonobservance of the end-March 2010 quantitative performance criteria on the overall budget deficit (excluding grants) and net government borrowing from the SBP on the grounds that their nonobservance was, in part, temporary (reflecting, among others, the delay in the disbursements of foreign financing), and adequate remedial actions have been agreed upon to address the remaining slippage. The criterion on the budget deficit was breached due to emergency spending pressures and revenue shortfalls, both reflecting to a large extent the unstable security situation. Shortfalls in IDP grants and Tokyo-related support from multilateral donors increased the margin of nonobservance via adjustors. Looking forward, the modified budget deficit target will allow the authorities to accommodate higher security spending. Also, external financing assumptions have been firmed up. Regarding the net borrowing from the SBP, the authorities have undertaken to improve government liquidity management and reduce its dependence on central bank financing. Staff also supports the requested rephasing of access and the modifications to the end-June performance criteria for overall budget deficit (excluding grants) and the net foreign assets of the SBP, which reflect understandings consistent with the principle of continued macroeconomic stabilization and the need to reduce external vulnerability. 
37. Staff recommends the completion of the fourth review. Security spending has put substantial pressure on the budget, and political and capacity constraints have affected the implementation of the structural agenda. Key reforms, however, their complexity and political challenges notwithstanding, reached the implementation stage. The most important of them - the parliamentary submission of the VAT legislative package - makes the introduction of the VAT possible in time for the next fiscal year, as reconfirmed recently by the authorities. Also, the needed reforms in tax administration moved ahead mitigating the possible downside risks associated with the VAT introduction. More needs to be done to ensure that other important structural reforms achieve a satisfactory momentum, including in the electricity sector and commodity operations. Given the authorities' successful track record in equally difficult areas, such as petroleum pricing and petroleum taxation and the liberalization of the foreign exchange market, staff remains confident that these complex and politically challenging reforms can be implemented. 
Figure 1. Pakistan: Selected Economic Indicators

(March 2009-March 2010, unless otherwise indicated)
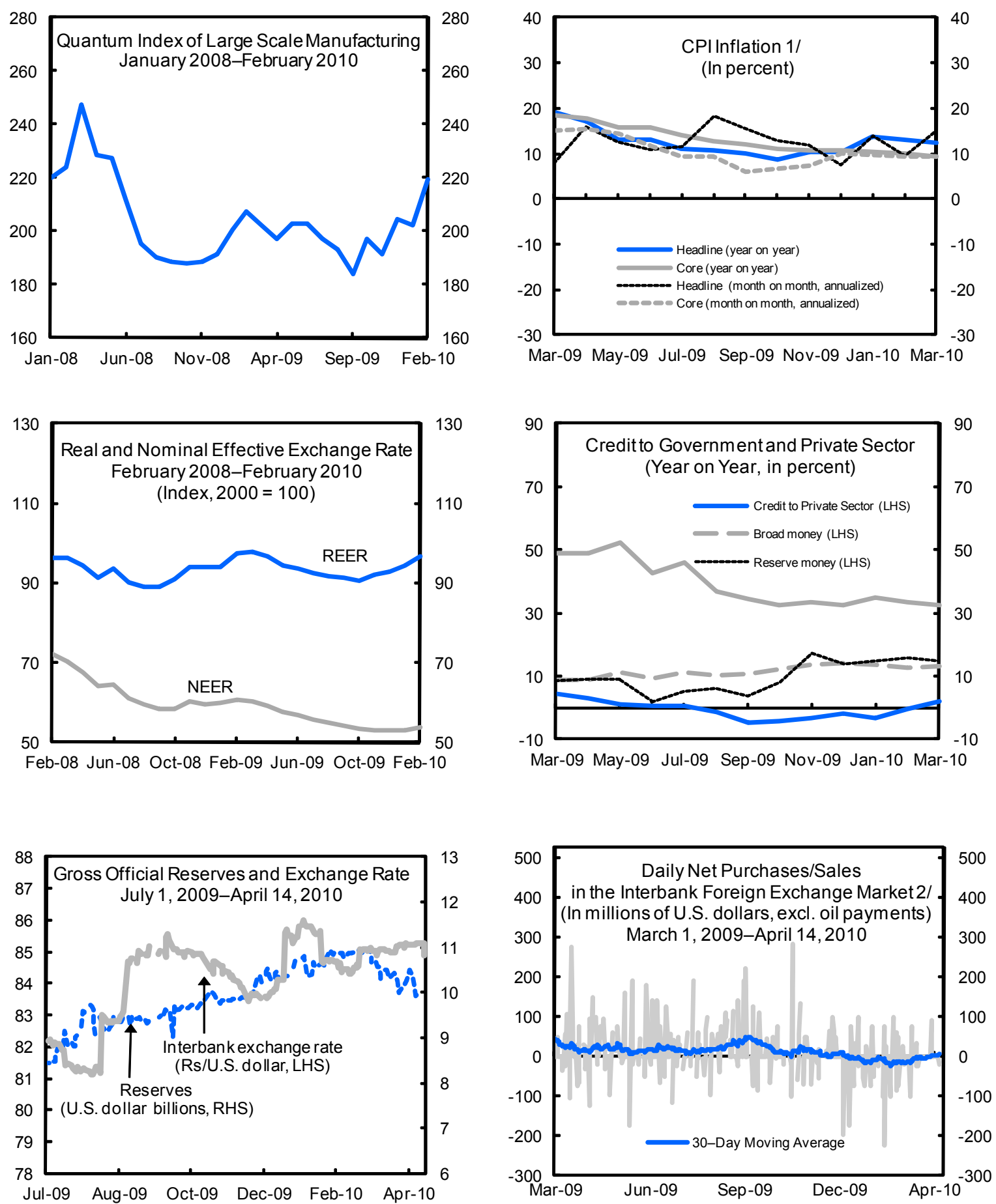

Source: Pakistani authorites.

1/Annualized month on month core and headline inflation are based on a three month moving
averages of the respective indices. 2/Positive values indicate netpurchases in the interbank market. 
Figure 2. Pakistan: Financial Market Indicators
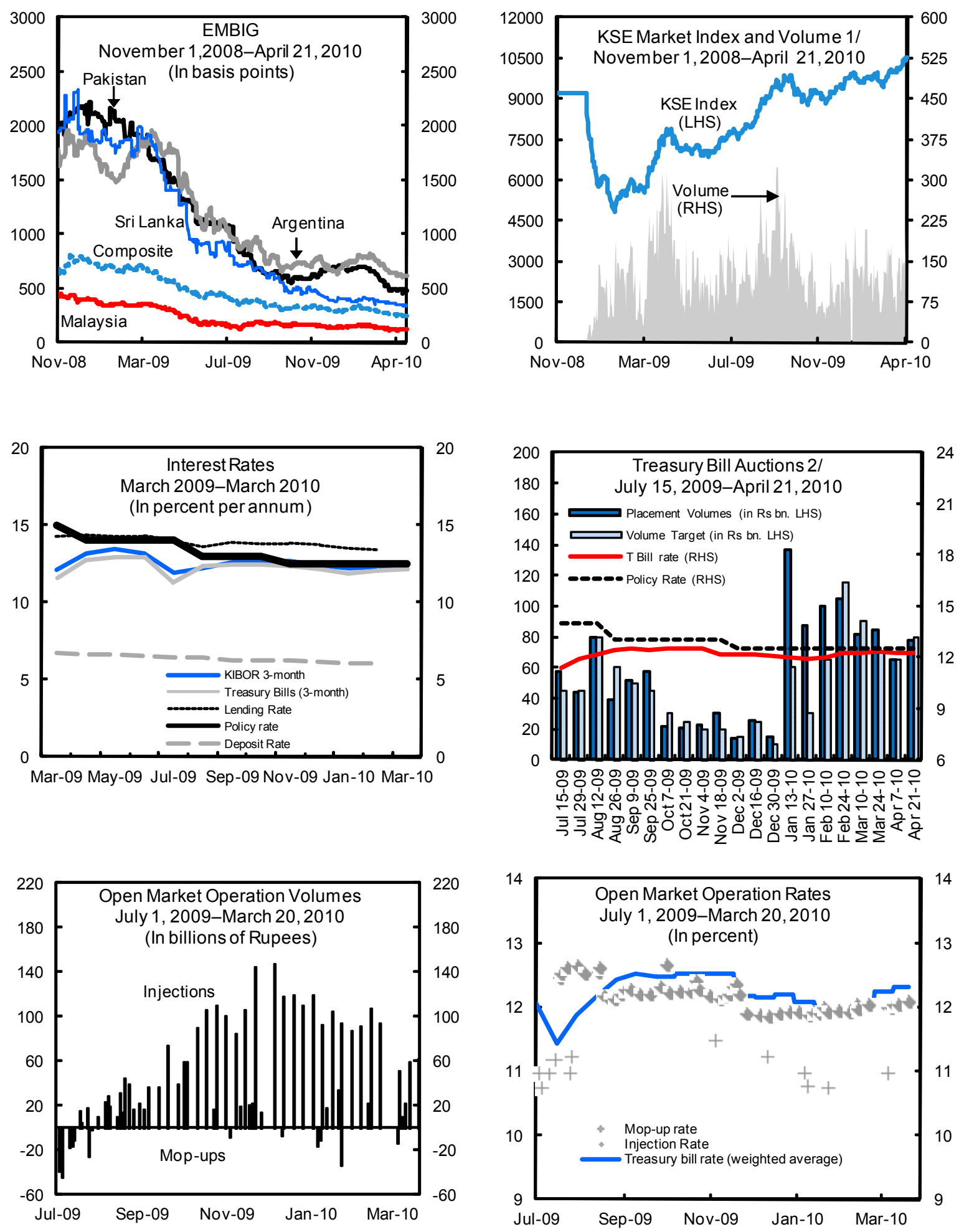

Sources: Pakistani authorities and Bloomberg.

$1 /$ Daily traded volumes are in millions of shares.

2/Placement volumes are for all maturities and the Treasury Bill rate is a weighted average. 
Figure 3. Pakistan: Real and External Sectors, 2006/07-2010/11
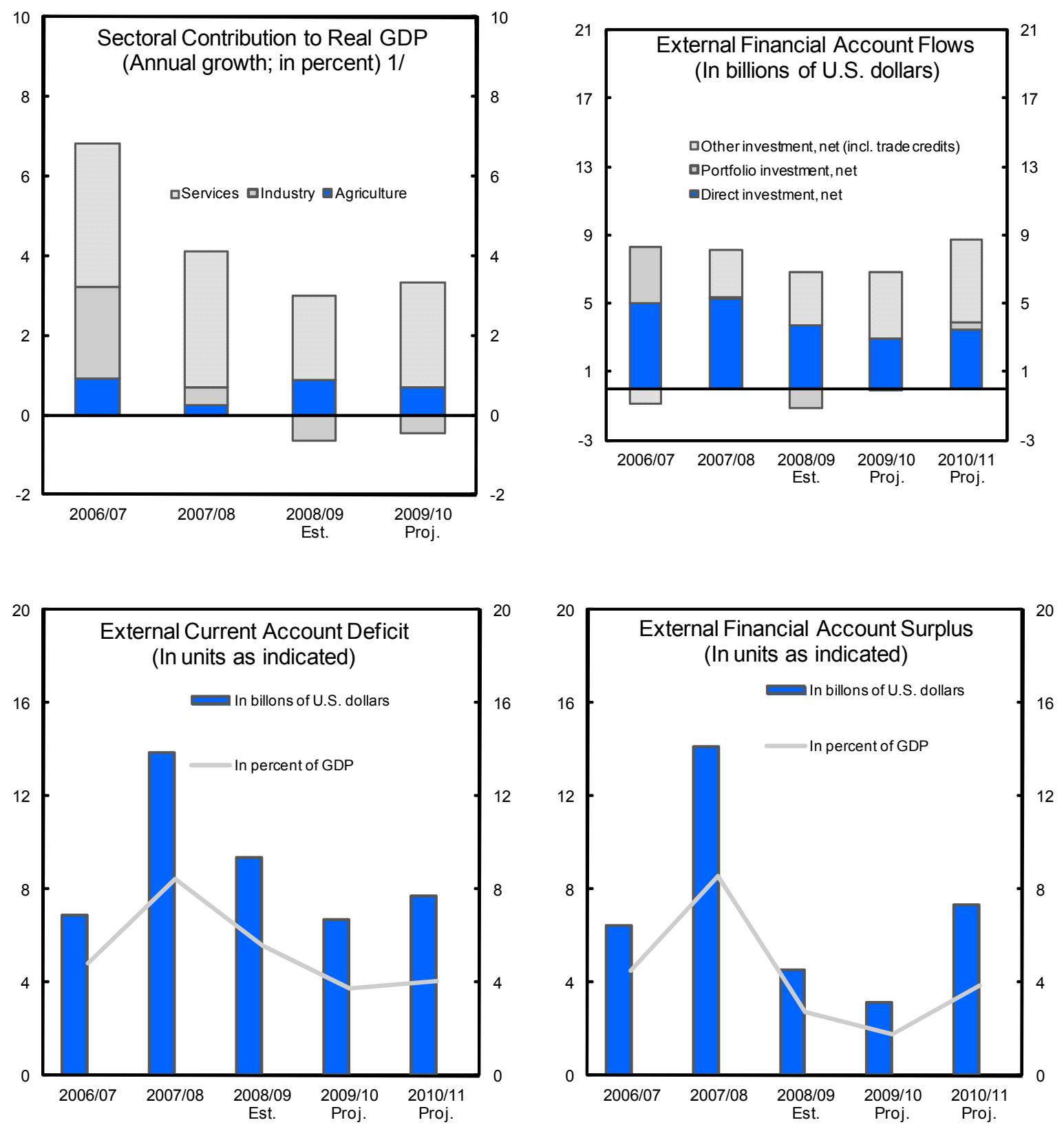

Sources: Pakistani authorities and Fund staff projections. 1/ At factor cost. 
Figure 4. Pakistan: Fiscal Policy Indicators
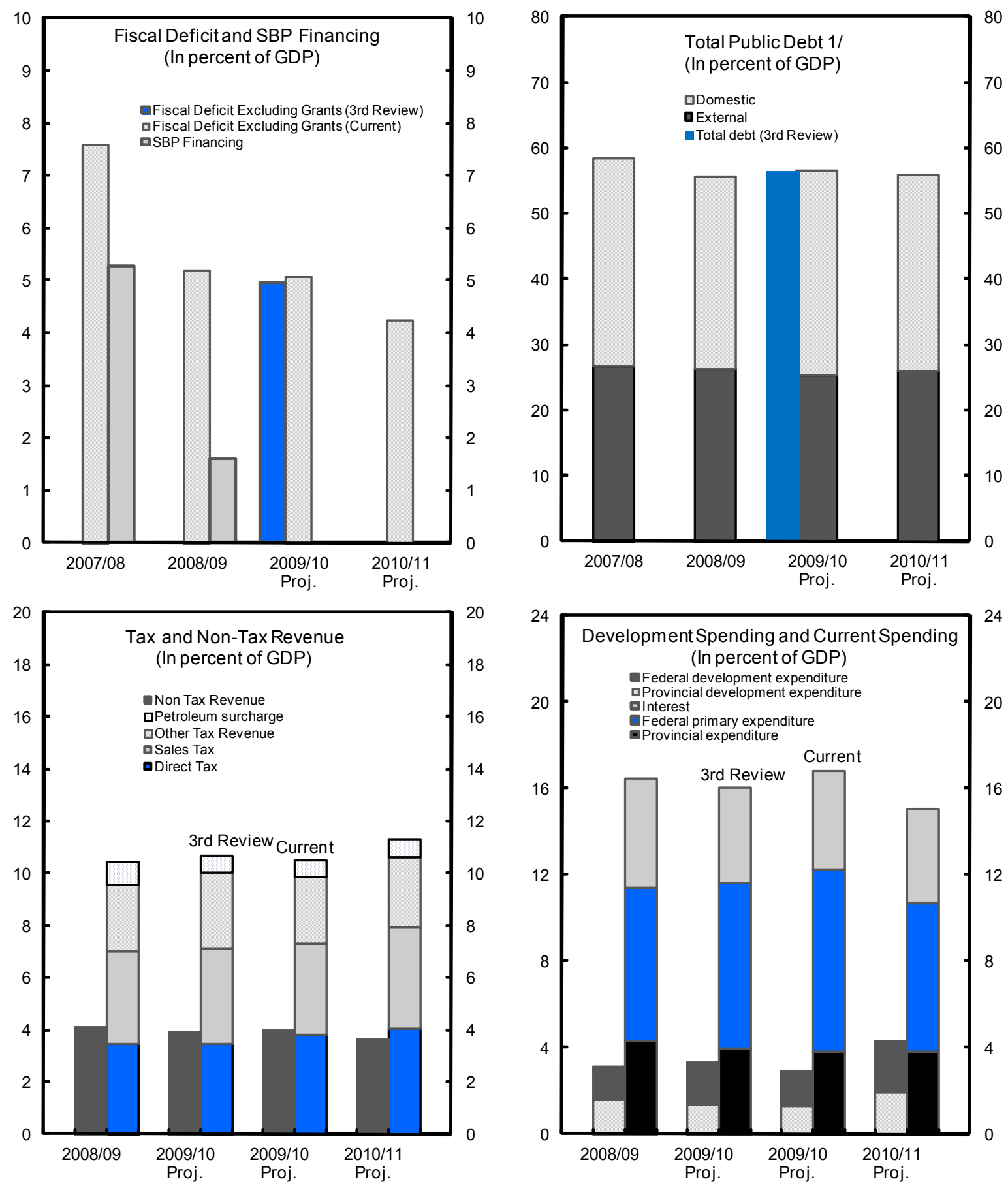

Sources: Pakistani authorities and Fund staff estimates and projections.

1/ Excluding IMF lending. 
Table 1. Pakistan: Selected Economic Indicators, 2007/08-2009/10 1/

(Population: 160.9 million (2007/08))

(Per capita GDP: US $\$ 1,042(2007 / 08))$

(Poverty rate: 17.2 percent $(2007 / 08))$

\begin{tabular}{|c|c|c|c|c|}
\hline & $2007 / 08$ & 2008/09 & $\frac{\text { Rev. Prog. } 2 /}{2009 / 1}$ & Proj. \\
\hline & \multicolumn{4}{|c|}{ (Annual percentage change) } \\
\hline \multicolumn{5}{|l|}{ Output and prices } \\
\hline Real GDP at factor cost & 4.1 & 2.0 & 3.0 & 3.0 \\
\hline Partner country demand (WEO definition) & 4.5 & & & \\
\hline Consumer prices (period average) & 12.0 & 20.8 & 11.0 & 11.5 \\
\hline Consumer prices (end of period) & 21.5 & 13.1 & 11.0 & 12.0 \\
\hline \multirow[t]{2}{*}{ Pakistani rupees per U.S. dollar (period average) } & 3.2 & 25.8 & $\ldots$ & $\ldots$ \\
\hline & \multicolumn{4}{|c|}{ (In percent of GDP) } \\
\hline Saving and investment & & & & \\
\hline Gross saving & 13.5 & 14.1 & 14.2 & 14.2 \\
\hline Government & -2.9 & -2.0 & -1.0 & -1.3 \\
\hline Nongovernment (including public sector enterprises) & 16.4 & 16.0 & 15.2 & 15.5 \\
\hline Gross capital formation 3/ & 22.0 & 19.7 & 18.5 & 17.9 \\
\hline Government & 4.4 & 3.0 & 3.3 & 3.2 \\
\hline Nongovernment (including public sector enterprises) & 17.6 & 16.7 & 15.1 & 14.7 \\
\hline \multicolumn{5}{|l|}{ Public finances } \\
\hline Revenue and grants & 14.9 & 14.3 & 15.2 & 14.9 \\
\hline Expenditure (including statistical discrepancy) & 22.2 & 19.3 & 19.6 & 19.5 \\
\hline Budget balance (including grants) & -7.3 & -5.0 & -4.3 & -4.6 \\
\hline Budget balance (including IDP grants) & & & -4.6 & -5.0 \\
\hline Budget balance (excluding grants) & -7.6 & -5.2 & -4.9 & -5.1 \\
\hline Primary balance & -2.5 & -0.1 & 0.1 & -0.2 \\
\hline Total government debt 4 / & 58.4 & 55.6 & 55.8 & 56.4 \\
\hline External government debt & 26.7 & 26.2 & 24.9 & 25.6 \\
\hline Domestic government debt & 31.8 & 29.4 & 30.9 & 30.8 \\
\hline \multirow[t]{2}{*}{ Total public debt (including all obligations to the IMF) } & 59.3 & 58.8 & $\ldots$ & 60.9 \\
\hline & \multicolumn{4}{|c|}{$\begin{array}{l}\text { (Annual changes in percent of initial stock of broad } \\
\text { money, unless otherwise indicated) }\end{array}$} \\
\hline Monetary sector & & & & \\
\hline Net foreign assets & -7.8 & -3.2 & 3.0 & 4.6 \\
\hline Net domestic assets & 23.2 & 12.8 & 9.0 & 9.7 \\
\hline Broad money & 15.3 & 9.6 & 12.0 & 14.3 \\
\hline Private credit (percentage change) & 16.4 & 0.7 & 11.6 & 11.6 \\
\hline Six-month treasury bill rate (period average, in percent) & 9.6 & 13.1 & $\ldots$ & $\ldots$ \\
\hline \multicolumn{5}{|l|}{ External sector } \\
\hline Merchandise exports, U.S. dollars (percentage change) & 18.2 & -6.4 & -2.9 & -0.8 \\
\hline Merchandise imports, U.S. dollars (percentage change) & 31.2 & -10.3 & -2.5 & -1.6 \\
\hline Current account balance (in percent of GDP) & -8.4 & -5.6 & -4.2 & -3.8 \\
\hline & \multicolumn{4}{|c|}{$\begin{array}{c}\text { (In percent of exports of goods and services, } \\
\text { unless otherwise indicated) }\end{array}$} \\
\hline External public and publicly guaranteed debt & 167.6 & 182.0 & 185.6 & 185.3 \\
\hline Debt service & 15.1 & 21.2 & 21.2 & 22.8 \\
\hline Implicit interest rate (in percent) $5 /$ & 2.7 & 2.4 & 2.4 & 2.2 \\
\hline Gross reserves (in millions of U.S. dollars) $6 /$ & 8,591 & 9,114 & 14,104 & 13,541 \\
\hline In months of next year's imports of goods and services & 2.6 & 2.8 & 4.2 & 4.0 \\
\hline \multicolumn{5}{|l|}{ Memorandum items: } \\
\hline Real effective exchange rate (annual average, percentage change) & -0.9 & -1.6 & $\ldots$ & \\
\hline Terms of trade (percentage change) & -10.2 & 14.0 & $\ldots$ & $\ldots$ \\
\hline Real per capita GDP (percentage change) & 2.3 & 0.3 & 1.3 & 1.3 \\
\hline GDP at market prices (in billions of Pakistani rupees) & 10,284 & 13,095 & 14,972 & 15,039 \\
\hline GDP at market prices (in billions of U.S. dollars) & 164.6 & 166.5 & 176.8 & 178.9 \\
\hline
\end{tabular}

Sources: Pakistani authorities; and Fund staff estimates and projections.

$1 /$ Fiscal year ends June 30.

2/ In all tables Revised Program refers to projections published in the report for the Third Review under the SBA (Country Report 10/6).

$3 /$ Including changes in inventories. Investment data recorded by the Pakistan Federal Bureau of Statistics are said

to underreport true activity.

4/ Excludes obligations to the IMF except budget financing, military debt, commercial loans, and short-term debt.

5/ Calculated as interest payments in percent of the end-of-period debt stock of the previous year.

6/ Excluding gold and foreign currency deposits of commercial banks held with the State Bank of Pakistan.

\section{CInternational Monetary Fund. Not for Redistribution}


Table 2. Pakistan: Balance of Payments, 2007/08-2009/10

(In millions of U.S. dollars; unless otherwise indicated)

\begin{tabular}{|c|c|c|c|c|c|c|c|c|c|}
\hline & \multirow[b]{3}{*}{$2007 / 08$} & \multirow[b]{3}{*}{ 2008/09 } & \multicolumn{2}{|c|}{ Prel. } & \multirow{2}{*}{$\frac{\text { Rev. Prog. }}{\text { Jan-Me }}$} & \multirow{2}{*}{$\begin{array}{ll} & \text { Proj. } \\
\text { ar } & \end{array}$} & \multirow{3}{*}{$\begin{array}{r}\text { Proj. } \\
\text { Apr-Jun }\end{array}$} & \multirow{3}{*}{$\frac{\text { Rev. Prog. }}{Y_{e}}$} & \multirow[t]{3}{*}{ Proj. } \\
\hline & & & Jul-Sep & Oct-Dec & & & & & \\
\hline & & & & & & $2009 / 10$ & & & \\
\hline Balance on goods & $-14,970$ & $-12,626$ & $-2,809$ & $-2,960$ & $-3,150$ & $-3,218$ & $-3,288$ & $-12,389$ & $-12,276$ \\
\hline Exports, f.o.b. & 20,427 & 19,121 & 4,620 & 4,699 & 4,650 & 4,779 & 4,865 & 18,562 & 18,964 \\
\hline Imports, f.o.b. & $-35,397$ & $-31,747$ & $-7,429$ & $-7,659$ & $-7,800$ & $-7,997$ & $-8,154$ & $-30,951$ & $-31,239$ \\
\hline Services (net) & $-6,457$ & $-3,473$ & -718 & -837 & -585 & -524 & -881 & $-2,959$ & $-2,960$ \\
\hline Services: credit & 3,589 & 4,014 & 838 & 1,040 & 1,350 & 1,193 & 1,517 & 4,485 & 4,588 \\
\hline Income (net) & $-3,923$ & $-4,403$ & -624 & -828 & $-1,150$ & -732 & $-1,525$ & $-4,616$ & $-3,709$ \\
\hline Income: credit & 1,613 & 873 & 106 & 137 & 230 & 164 & 179 & 883 & 586 \\
\hline Income: debit & $-5,536$ & $-5,276$ & -730 & -965 & $-1,380$ & -896 & $-1,704$ & $-5,499$ & $-4,295$ \\
\hline Of which: interest payments & $-2,161$ & $-1,908$ & -268 & -450 & -431 & -467 & -692 & $-1,850$ & $-1,877$ \\
\hline Of which: income on direct investment & $-3,375$ & $-3,368$ & -438 & -492 & -949 & -429 & $-1,059$ & $-3,649$ & $-2,418$ \\
\hline Balance on goods, services, and income & $-25,350$ & $-20,502$ & $-4,151$ & $-4,625$ & $-4,885$ & $-4,475$ & $-5,694$ & $-19,964$ & $-18,945$ \\
\hline Current transfers (net) & 11,476 & 11,163 & 3,619 & 3,143 & 3,163 & 2,871 & 2,601 & 12,492 & 12,234 \\
\hline Current transfers: credit, of which: & 11,618 & 11,265 & 3,658 & 3,157 & 3,180 & 2,895 & 2,609 & 12,550 & 12,319 \\
\hline Official & 484 & 219 & 11 & 124 & 155 & 30 & 14 & 300 & 179 \\
\hline Workers' remittances & 6,451 & 7,811 & 2,331 & 2,199 & 2,125 & 1,977 & 1,869 & 8,500 & 8,375 \\
\hline Current transfers: debit & -142 & -102 & -39 & -14 & -16 & -24 & -8 & -58 & -85 \\
\hline Capital account & 121 & 474 & 38 & 48 & 461 & 510 & 692 & 1,146 & 1,288 \\
\hline Capital transfers: credit & 128 & 479 & 38 & 53 & 464 & 509 & 691 & 1,149 & 1,291 \\
\hline Of which: official capital grants & 111 & 427 & 27 & 42 & 460 & 330 & 306 & 1,134 & 705 \\
\hline Capital transfers: debit & -7 & -5 & 0 & -5 & -3 & 1 & 1 & -3 & -3 \\
\hline Financial account & 8,131 & 5,720 & 1,897 & 116 & 2,039 & 963 & 3,816 & 7,630 & 6,792 \\
\hline Direct investment abroad & -75 & -25 & 36 & 0 & -20 & -1 & -75 & -30 & -40 \\
\hline Direct investment in Pakistan & 5,410 & 3,720 & 472 & 541 & 650 & 593 & 1,348 & 2,666 & 2,954 \\
\hline Of which: privatization receipts & 133 & 0 & 0 & 0 & 133 & 0 & 800 & 266 & 800 \\
\hline Portfolio investment (net), of which: & 32 & $-1,096$ & 202 & 60 & -475 & -800 & 454 & -34 & -84 \\
\hline Eurobond/GDR & -25 & -544 & -22 & 0 & -600 & -600 & 0 & -622 & -622 \\
\hline Other investment assets & 32 & 560 & -235 & -265 & -104 & -53 & -93 & -284 & -646 \\
\hline General government & 5 & 8 & 2 & -2 & -4 & -3 & -5 & -8 & -8 \\
\hline Banks & 525 & 346 & 60 & 94 & -50 & -150 & -204 & -200 & -200 \\
\hline Other sectors & -498 & 206 & -168 & -124 & -50 & 100 & 116 & -76 & -76 \\
\hline Other investment liabilities & 2,732 & 2,561 & 1,422 & -220 & 1,988 & 1,224 & 2,182 & 5,312 & 4,608 \\
\hline Monetary authorities & 490 & -1 & 1,250 & 0 & 0 & 0 & 0 & 1,250 & 1,250 \\
\hline General government, of which: & 1,761 & 1,969 & 111 & -225 & 1,381 & 370 & 1,885 & 2,271 & 2,141 \\
\hline Disbursements & 3,054 & 3,642 & 617 & 762 & 1,741 & 849 & 2,559 & 4,448 & 4,787 \\
\hline Other sectors & 415 & 302 & -35 & 82 & 551 & 826 & 243 & 1,591 & 1,116 \\
\hline Net errors and omissions & -166 & -89 & -324 & 287 & 0 & 0 & 0 & -522 & -36 \\
\hline Reserves and related items & 5,788 & 3,234 & $-1,080$ & 1,031 & -778 & 130 & $-1,414$ & -782 & $-1,333$ \\
\hline Reserve assets, of which: & 5,961 & -457 & $-2,154$ & -90 & $-1,900$ & 156 & $-2,485$ & $-5,140$ & $-4,574$ \\
\hline Foreign exchange (State Bank of Pakistan) & 5,711 & -523 & $-2,103$ & -61 & $-1,800$ & 193 & $-2,455$ & $-4,990$ & $-4,427$ \\
\hline Foreign exchange (deposit money banks) & 250 & 66 & -51 & -29 & -100 & -37 & -30 & -150 & -147 \\
\hline Use of Fund credit and loans & -173 & 3,691 & 1,174 & 1,121 & 1,122 & -26 & 1,071 & 4,358 & 3,341 \\
\hline Exceptional financing & 0 & 0 & -100 & 0 & 0 & 0 & 0 & 0 & -100 \\
\hline \multicolumn{10}{|l|}{ Memorandum items: } \\
\hline Current account (in percent of GDP) & -8.4 & -5.6 & -0.3 & -0.8 & -1.0 & -0.9 & -1.7 & -4.2 & -3.8 \\
\hline Current account (in percent of GDP; excluding fuel imports) & -2.1 & 0.4 & $\ldots$ & $\ldots$ & $\ldots$ & $\ldots$ & $\ldots$ & 1.9 & 2.1 \\
\hline Exports f.o.b. (growth rate, in percent) & 18.2 & -6.4 & $\ldots$ & $\ldots$ & $\ldots$ & $\ldots$ & $\ldots$ & -2.9 & -0.8 \\
\hline Imports f.o.b. (growth rate, in percent) & 31.2 & -10.3 & $\ldots$ & $\ldots$ & $\ldots$ & $\ldots$ & $\ldots$ & -2.5 & -1.6 \\
\hline Imports f.o.b. (growth rate, in percent, excluding oil) & 26.8 & -12.2 & $\ldots$ & $\ldots$ & $\ldots$ & $\ldots$ & $\ldots$ & -7.1 & -3.7 \\
\hline Oil imports (in million US\$, cif) & 10,496 & 10,031 & $\ldots$ & $\ldots$ & $\ldots$ & $\ldots$ & $\ldots$ & 10,888 & 10,389 \\
\hline Crude oil price (\$/bbl) & 87.0 & 68.5 & $\ldots$ & $\ldots$ & $\ldots$ & $\ldots$ & $\ldots$ & 74.3 & 73.6 \\
\hline Terms of trade (growth rate, in percent) & -10.2 & $\ldots$ & $\ldots$ & $\ldots$ & $\ldots$ & $\ldots$ & $\ldots$ & $\ldots$ & \\
\hline External debt (in millions of U.S. dollars) & 44,468 & 50,759 & 53,066 & 52,884 & 57,061 & 55,913 & 55,774 & 57,061 & 55,774 \\
\hline Gross financing needs (in millions of U.S. dollars) $1 /$ & 15,228 & 12,295 & $\ldots$ & $\ldots$ & $\ldots$ & $\ldots$ & $\ldots$ & 10,408 & 10,211 \\
\hline End-period gross official reserves (millions of U.S. dollars) 2/ & 8,591 & 9,114 & 11,214 & 11,274 & 12,714 & 11,081 & 13,541 & 14,104 & 13,541 \\
\hline (In months of next year's imports of goods and services) & 2.6 & 2.8 & $\ldots$ & $\ldots$ & $\ldots$ & $\ldots$ & $\ldots$ & 4.2 & 4.0 \\
\hline (in percent of debt service) & 244.4 & 187.4 & $\ldots$ & $\ldots$ & $\ldots$ & $\ldots$ & $\ldots$ & 294.7 & 277.7 \\
\hline GDP (in millions of U.S. dollars) & 164,557 & 166,515 & 178,922 & 178,922 & 176,835 & 178,922 & 178,922 & 176,835 & 178,922 \\
\hline
\end{tabular}

Sources: Pakistani authorities; and Fund staff estimates and projections.

1/ Defined as current account deficit, plus amortization on medium- and long-term debt, plus short-term debt at end of previous period.

2/ Excluding foreign currency deposits held with the State Bank of Pakistan (cash reserve requirements) and gold. 
Table 3a. Pakistan: Consolidated Government Budget, 2007/08-2009/10 (In billions of Pakistani rupees)

\begin{tabular}{|c|c|c|c|c|c|c|c|c|c|c|}
\hline & & & $\frac{\text { Actual }}{\text { Jul-Sep }}$ & $\begin{array}{r}\text { Prel. } \\
\text { Oct-Dec }\end{array}$ & $\frac{\text { Rev. Prog. }}{\text { Jan-Mar }}$ & Proj. & $\frac{\text { Proj. 1/ }}{\text { Apr-Jun }}$ & Budget & Rev. Prog. & Proj. \\
\hline & $2007 / 08$ & $2008 / 09$ & \multicolumn{8}{|c|}{$2009 / 10$} \\
\hline Revenue and grants & 1,530 & 1,877 & 430 & 488 & 604 & 595 & 733 & 2,222 & 2,280 & 2,247 \\
\hline Revenue & 1,499 & 1,851 & 427 & 478 & 568 & 561 & 706 & 2,156 & 2,187 & 2,172 \\
\hline Tax revenue & 1,086 & 1,331 & 305 & 364 & 398 & 410 & 486 & 1,593 & 1,600 & 1,565 \\
\hline Federal & 1,045 & 1,285 & 293 & 352 & 379 & 397 & 472 & 1,523 & 1,530 & 1,515 \\
\hline FBR revenue & 1,007 & 1,157 & 263 & 320 & 344 & 362 & 435 & 1,380 & 1,396 & 1,380 \\
\hline Direct taxes & 388 & 440 & 84 & 127 & 124 & 151 & 178 & 566 & 522 & 540 \\
\hline Federal excise duty & 84 & 116 & 28 & 28 & 40 & 31 & 47 & 153 & 161 & 134 \\
\hline Sales tax & 385 & 452 & 117 & 126 & 136 & 138 & 160 & 499 & 547 & 540 \\
\hline Customs duties & 151 & 148 & 33 & 38 & 44 & 43 & 50 & 162 & 166 & 165 \\
\hline Petroleum surcharge & 14 & 112 & 24 & 28 & 27 & 25 & 25 & 112 & 102 & 102 \\
\hline Gas surcharge and other & 23 & 16 & 7 & 5 & 8 & 10 & 12 & 31 & 31 & 33 \\
\hline Provincial & 41 & 46 & 11 & 12 & 19 & 13 & 14 & 70 & 70 & 50 \\
\hline Nontax revenue & 414 & 520 & 123 & 114 & 170 & 151 & 220 & 563 & 588 & 608 \\
\hline Federal & 336 & 436 & 113 & 107 & 140 & 131 & 186 & 468 & 493 & 536 \\
\hline Provincial & 78 & 84 & 10 & 7 & 30 & 20 & 35 & 95 & 95 & 72 \\
\hline Grants & 31 & 26 & 3 & 10 & 36 & 34 & 27 & 65 & 92 & 74 \\
\hline Of which: Tokyo pledges & $\ldots$ & $\ldots$ & 0 & 0 & 11 & 25 & 21 & 46 & 28 & 46 \\
\hline Of which: IDP grants & $\ldots$ & $\ldots$ & 0 & 4 & 20 & 0 & 0 & 39 & 45 & 4 \\
\hline Expenditure & 2,281 & 2,497 & 637 & 668 & 772 & 797 & 837 & 2,877 & 2,913 & 2,939 \\
\hline Current expenditure & 1,858 & 2,093 & 544 & 561 & 625 & 651 & 677 & 2,261 & 2,403 & 2,433 \\
\hline Federal & 1,420 & 1,547 & 405 & 416 & 478 & 511 & 552 & 1,671 & 1,813 & 1,883 \\
\hline Interest & 490 & 638 & 141 & 153 & 159 & 168 & 198 & 647 & 659 & 660 \\
\hline Domestic & $\ldots$ & 559 & 129 & 133 & 140 & 145 & 170 & 577 & 577 & 577 \\
\hline Foreign & $\ldots$ & 79 & 12 & 20 & 19 & 23 & 28 & 70 & 83 & 83 \\
\hline Other & 931 & 909 & 263 & 263 & 319 & 343 & 354 & 1,024 & 1,153 & 1,223 \\
\hline Of which: subsidies & $\ldots$ & 244 & 46 & 31 & 66 & 66 & 55 & 132 & 197 & 197 \\
\hline Of which: grants $1 /$ & $\ldots$ & 136 & 81 & 98 & 95 & 115 & 120 & 314 & 344 & 414 \\
\hline Of which: Cash transfers to poor households $2 /$ & $\ldots$ & 21 & 10 & 13 & 25 & 19 & 22 & 84 & 84 & 64 \\
\hline Provincial & 437 & 546 & 140 & 145 & 148 & 140 & 125 & 590 & 590 & 550 \\
\hline Development expenditure and net lending & 423 & 404 & 93 & 107 & 147 & 147 & 160 & 617 & 510 & 505 \\
\hline Public Sector Development Program & 452 & 398 & 85 & 99 & 146 & 144 & 157 & 606 & 500 & 485 \\
\hline Federal & 238 & 196 & 54 & 57 & 94 & 85 & 89 & 406 & 300 & 285 \\
\hline Of which: One-off expenditure & 17 & 27 & 6 & 6 & 6 & 6 & 6 & 0 & 25 & 25 \\
\hline Provincial & 214 & 202 & 32 & 42 & 52 & 59 & 68 & 200 & 200 & 200 \\
\hline Net lending & -28 & 7 & 8 & 8 & 1 & 3 & 3 & 11 & 11 & 21 \\
\hline Statistical discrepancy (" $+"=$ additional expenditure) $3 /$ & -3 & 34 & 16 & -13 & 0 & -3 & -4 & 0 & 14 & -4 \\
\hline Overall Deficit Ceiling (excluding grants) & -779 & -680 & -225 & -177 & -204 & -234 & -126 & -721 & -740 & -762 \\
\hline Overall Deficit (including IDP grants) & & & -225 & -172 & -199 & -234 & -126 & & -695 & -758 \\
\hline Overall Deficit (including all grants) & -748 & -655 & -222 & -167 & -168 & -200 & -99 & -656 & -648 & -688 \\
\hline Financing & 748 & 655 & 222 & 167 & 168 & 200 & 99 & 656 & 648 & 688 \\
\hline External & 121 & 72 & 75 & 22 & 109 & -40 & 218 & 312 & 232 & 276 \\
\hline Of which: Tokyo pledges & $\ldots$ & $\ldots$ & 0 & 44 & 51 & 13 & 31 & $\ldots$ & 160 & 87 \\
\hline Of which: IMF & $\ldots$ & $\ldots$ & 61 & 31 & 30 & 0 & -36 & $\ldots$ & 0 & 57 \\
\hline Domestic & 628 & 583 & 147 & 144 & 59 & 240 & -119 & 343 & 415 & 412 \\
\hline Bank & 520 & 356 & 39 & 68 & 12 & 104 & -39 & 97 & 194 & 172 \\
\hline Nonbank & 106 & 227 & 108 & 76 & 47 & 136 & -81 & 246 & 240 & 240 \\
\hline \multicolumn{11}{|l|}{ Memorandum items: } \\
\hline Expenditure 4/ & 2,295 & 2,558 & 659 & 661 & 778 & 801 & 839 & 2,877 & 2,953 & 2,959 \\
\hline Primary balance (excluding grants) & -289 & -43 & -84 & -24 & -46 & -66 & 72 & -74 & -81 & -102 \\
\hline Primary balance (including grants) & -259 & -17 & -81 & -14 & -9 & -32 & 99 & -8 & 12 & -28 \\
\hline Augmented fiscal balance (excluding grants) $5 /$ & $\ldots$ & $\ldots$ & -441 & -238 & -204 & -234 & -126 & $\ldots$ & $-1,017$ & $-1,039$ \\
\hline Defense spending & 285 & 424 & 136 & 138 & 121 & 154 & 179 & 448 & 513 & 593 \\
\hline Total government debt $6 /$ & 6,010 & 7,282 & $\ldots$ & $\ldots$ & $\ldots$ & $\ldots$ & $\ldots$ & $\ldots$ & 8,439 & 8,488 \\
\hline Domestic debt & 3,266 & 3,853 & $\ldots$ & $\ldots$ & $\ldots$ & $\ldots$ & $\ldots$ & $\ldots$ & 4,632 & 4,631 \\
\hline External debt $6 /$ & 2,744 & 3,430 & $\ldots$ & $\ldots$ & $\ldots$ & $\ldots$ & $\ldots$ & $\ldots$ & 3,807 & 3,857 \\
\hline Nominal GDP (market prices) & 10,284 & 13,095 & 15,039 & 15,039 & 14,972 & 15,039 & 15,039 & 14,972 & 14,972 & 15,039 \\
\hline
\end{tabular}

Sources: Pakistani authorities for historical data; and Fund staff for estimates and projections.

1/ Figures for revenue, grants, and expenditure are based on the projections agreed with the authorities in February 2010.

Figures for deficit (excluding grants) and domestic bank and nonbank financing are based on preliminary estimates through March 2010. 2/ For 2009/10 projections, this figure includes Rs. 145 bn on security operations.

3/ Comprises BISP, Bait-ul-Mal, and Pakistan Poverty Alleviation Fund.

4/ The statistical discrepancy is believed to arise mainly from double-counting of spending at the provincial level.

$5 /$ Includes statistical discrepancy and spending related to the 2005 earthquake.

6/ Reflects assumption of electricity sector debt by the budget.

7/ Excludes obligations to the IMF except budget financing, military debt, commercial loans, and short-term debt. 
Table 3b. Pakistan: Consolidated Government Budget, 2007/08-2009/10 (In percent of GDP; unless otherwise indicated)

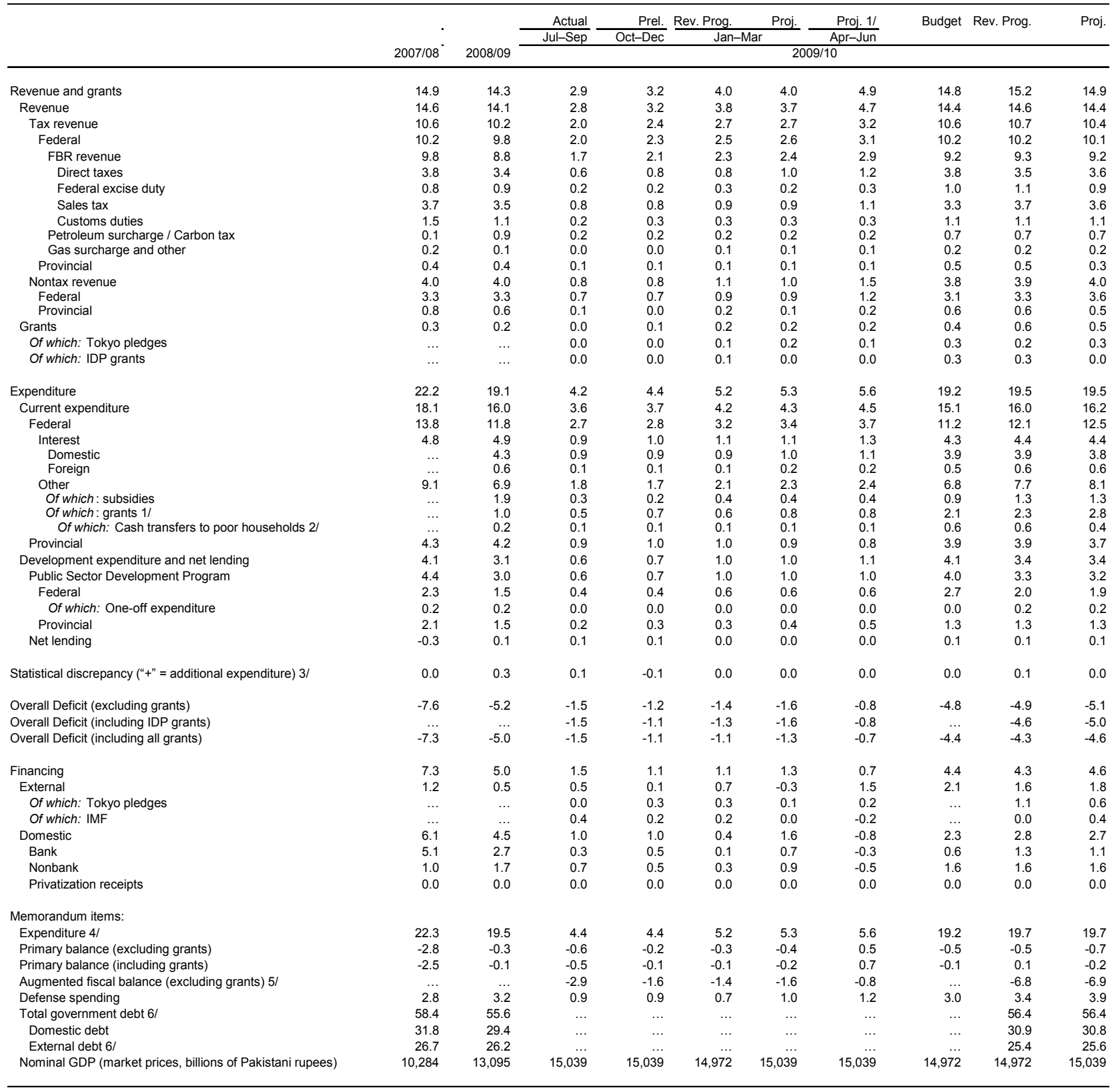

Sources: Pakistani authorities for historical data; and Fund staff for estimates and projections.

1/ Figures for revenue, grants, and expenditure are based on the projections agreed with the authorities in February 2010. Figures for deficit (excluding grants) and domestic bank and nonbank financing are based on preliminary estimates through March 2010. 2/ For 2009/10 projections, this figure includes Rs. 145 bn on security operations.

3/ Comprises BISP, Bait-ul-Mal, and Pakistan Poverty Alleviation Fund.

4/ The statistical discrepancy is believed to arise mainly from double-counting of spending at the provincial level.

$5 /$ Includes statistical discrepancy and spending related to the 2005 earthquake.

$6 /$ Reflects assumption of electricity sector debt by the budget.

7/ Excludes obligations to the IMF except budget financing, military debt, commercial loans, and short-term debt. 
Table 4. Pakistan: Monetary Survey and Analytical Balance Sheet of the State Bank of Pakistan, 2007/08-2009/10

\begin{tabular}{|c|c|c|c|c|c|c|c|}
\hline & \multirow[b]{3}{*}{$2007 / 08$} & \multirow[b]{3}{*}{$2008 / 09$} & Act. & Act. & Act. & Rev. Prog. & Proj. \\
\hline & & & Sep. & Dec. & Mar. & \multicolumn{2}{|l|}{ Jun. } \\
\hline & & & \multicolumn{5}{|c|}{$2009 / 10$} \\
\hline & \multicolumn{7}{|c|}{ (In billions of Pakistani rupees) } \\
\hline \multicolumn{8}{|l|}{ Monetary survey } \\
\hline Net foreign assets (NFA) & 668 & 517 & 658 & 628 & 578 & 671 & 754 \\
\hline Net domestic assets (NDA) & 4,022 & 4,620 & 4,517 & 4,849 & 4,869 & 5,082 & 5,120 \\
\hline Net claims on government, of which: & 1,473 & 1,997 & 2,032 & 2,118 & 2,169 & 2,121 & 2,132 \\
\hline Budget support, of which: & 1,325 & 1,630 & 1,669 & 1,737 & 1,841 & 1,805 & 1,802 \\
\hline Banks & 310 & 500 & 622 & 671 & 681 & 676 & 672 \\
\hline Commodity operations & 127 & 336 & 335 & 328 & 270 & 336 & 351 \\
\hline Credit to nongovernment & 3,018 & 3,190 & 3,180 & 3,407 & 3,425 & 3,680 & 3,685 \\
\hline Private sector & 2,904 & 2,924 & 2,849 & 3,047 & 3,071 & 3,263 & 3,264 \\
\hline Public sector enterprises & 114 & 266 & 331 & 359 & 354 & 416 & 421 \\
\hline Privatization account & -3 & -3 & -3 & -3 & -3 & -3 & -3 \\
\hline Other items, net & -466 & -564 & -692 & -673 & -722 & -716 & -695 \\
\hline Broad money & 4,689 & 5,137 & 5,176 & 5,477 & 5,447 & 5,753 & 5,874 \\
\hline Currency outside scheduled banks & 982 & 1,152 & 1,261 & 1,297 & 1,277 & 1,308 & 1,326 \\
\hline Rupee deposits & 3,443 & 3,705 & 3,614 & 3,861 & 3,840 & 4,126 & 4,214 \\
\hline Foreign currency deposits & 263 & 280 & 301 & 319 & 330 & 319 & 334 \\
\hline \multicolumn{8}{|l|}{ State Bank of Pakistan (SBP) } \\
\hline NFA & 480 & 324 & 475 & 452 & 399 & 470 & 500 \\
\hline NDA & 1,000 & 1,183 & 1,141 & 1,209 & 1,231 & 1,262 & 1,254 \\
\hline Net claims on government & 1,015 & 1,144 & 1,058 & 1,080 & 1,172 & 1,144 & 1,144 \\
\hline Of which: budget support & 1,016 & 1,130 & 1,047 & 1,066 & 1,160 & 1,130 & 1,130 \\
\hline Claims on nongovernment & -7 & -7 & -7 & -8 & -6 & -7 & -7 \\
\hline Claims on scheduled banks & 227 & 303 & 297 & 327 & 322 & 353 & 363 \\
\hline Privatization account & -3 & -3 & -3 & -3 & -3 & -3 & -3 \\
\hline Other items, net & -231 & -254 & -204 & -188 & -254 & -226 & -243 \\
\hline Reserve money, of which: & 1,480 & 1,508 & 1,616 & 1,660 & 1,630 & 1,732 & 1,754 \\
\hline Banks' reserves & 425 & 274 & 267 & 281 & 267 & 341 & 346 \\
\hline Currency & 1,051 & 1,229 & 1,343 & 1,374 & 1,357 & 1,385 & 1,403 \\
\hline & \multicolumn{7}{|c|}{ (Annual percentage change, unless otherwise indicated) } \\
\hline Broad money & 15.3 & 9.6 & 10.7 & 14.3 & 13.4 & 12.0 & 14.3 \\
\hline NFA, banking system (in percent of broad money) $1 /$ & -7.8 & -3.2 & 3.7 & 5.3 & 2.4 & 3.0 & 4.6 \\
\hline NDA, banking system (in percent of broad money) $1 /$ & 23.2 & 12.8 & 7.0 & 9.0 & 11.0 & 9.0 & 9.7 \\
\hline Budgetary support (in percent of broad money) $1 /$ & 12.5 & 6.5 & 5.1 & 4.8 & 7.1 & 3.4 & 3.3 \\
\hline NFA, banking system & -32.2 & -22.5 & 35.6 & 67.3 & 24.9 & 29.8 & 45.7 \\
\hline NDA, banking system & 30.6 & 14.9 & 7.9 & 9.8 & 12.1 & 10.0 & 10.8 \\
\hline Budgetary support & 62.4 & 23.0 & 16.7 & 15.3 & 22.6 & 10.8 & 10.5 \\
\hline Private credit & 16.4 & 0.7 & -4.9 & -1.9 & 3.1 & 11.6 & 11.6 \\
\hline Currency & 16.9 & 17.3 & 13.9 & 15.4 & 14.6 & 13.6 & 15.1 \\
\hline Reserve money & 22.3 & 1.9 & 3.7 & 14.0 & 12.2 & 14.9 & 16.3 \\
\hline NFA, SBP (in percent of reserve money) $1 /$ & -25.4 & -10.5 & 10.7 & 18.2 & 10.1 & 9.7 & 11.6 \\
\hline NDA, SBP (in percent of reserve money) $1 /$ & 47.7 & 12.4 & -7.0 & -4.2 & 2.0 & 5.2 & 4.7 \\
\hline Net claims on government (in percent of reserve money) $1 /$ & 56.9 & 8.6 & -11.3 & -9.4 & 4.8 & 0.0 & 0.0 \\
\hline \multicolumn{8}{|l|}{ Memorandum items: } \\
\hline Velocity & 2.2 & 2.5 & $\ldots$ & $\ldots$ & $\ldots$ & 2.6 & 2.6 \\
\hline Money multiplier & 3.2 & 3.4 & 3.2 & 3.3 & 3.3 & 3.3 & 3.3 \\
\hline Currency to broad money ratio (percent) & 20.9 & 22.4 & 24.4 & 23.7 & 23.4 & 22.7 & 22.6 \\
\hline Currency to deposit ratio (percent) & 26.5 & 28.9 & 32.2 & 31.0 & 30.6 & 29.4 & 29.2 \\
\hline Foreign currency to deposit ratio (percent) & 7.1 & 7.0 & 7.7 & 7.6 & 7.9 & 7.2 & 7.3 \\
\hline Reserves to deposit ratio (percent) & 11.5 & 6.9 & 6.8 & 6.7 & 6.4 & 7.7 & 7.6 \\
\hline Budget bank financing (billions of Pakistani rupees), of which: & 509 & 305 & 39 & 107 & 211 & 175 & 172 \\
\hline By commercial banks & -167 & 191 & 122 & 171 & 181 & 175 & 172 \\
\hline By SBP & 677 & 114 & -83 & -64 & 30 & 0 & 0 \\
\hline NFA of SBP (change from beginning of the year in billions of U.S. dollars) 2/ & -4.5 & -3.1 & 1.7 & 1.4 & 0.8 & 1.4 & 1.8 \\
\hline NFA of commercial banks (millions of U.S. dollars) & 2,748 & 2,370 & 2,212 & 2,099 & 2,120 & 2,367 & 2,919 \\
\hline NDA of commercial banks (billions of Pakistani rupees) & 3,022 & 3,437 & 3,376 & 3,640 & 3,639 & 3,820 & 3,866 \\
\hline Excess reserves in percent of broad money & 1.5 & 0.3 & 0.0 & 0.0 & -0.3 & $\ldots$ & $\ldots$ \\
\hline
\end{tabular}

Sources: Pakistani authorities for historical data; and Fund staff estimates and projections.

$1 /$ Denominator is the stock of broad (reserve) money at the end of the previous year.

2/ Includes valuation adjustments. 
Table 5. Pakistan: Medium-Term Macroeconomic Framework, 2007/08-2014/15

\begin{tabular}{|c|c|c|c|c|c|c|c|c|}
\hline & \multirow[b]{2}{*}{$2007 / 08$} & \multirow[b]{2}{*}{$2008 / 09$} & \multicolumn{6}{|c|}{ Projections } \\
\hline & & & $2009 / 10$ & $2010 / 11$ & $2011 / 12$ & $2012 / 13$ & $2013 / 14$ & $2014 / 15$ \\
\hline & \multicolumn{8}{|c|}{ (Annual changes in percent) } \\
\hline \multicolumn{9}{|l|}{ Output and prices } \\
\hline Real GDP at factor cost & 4.1 & 2.0 & 3.0 & 4.0 & 4.5 & 5.0 & 5.5 & 6.0 \\
\hline Consumer prices (period average) & 12.0 & 20.8 & 11.5 & 7.5 & 6.5 & 6.0 & 6.0 & 6.0 \\
\hline & \multicolumn{8}{|c|}{ (In percent of GDP) } \\
\hline Saving and investment balance & -8.4 & -5.6 & -3.8 & -4.0 & -3.9 & -3.6 & -3.4 & -3.4 \\
\hline Government & -7.3 & -5.0 & -4.6 & -3.8 & -3.1 & -2.8 & -2.7 & -2.0 \\
\hline Non-government (including public sector enterprises) & -1.2 & -0.6 & 0.8 & -0.2 & -0.8 & -0.8 & -0.8 & -1.4 \\
\hline Gross national saving & 13.5 & 14.1 & 14.2 & 17.0 & 17.5 & 18.3 & 19.5 & 20.8 \\
\hline Government & -2.9 & -2.0 & -1.3 & 0.6 & 1.4 & 1.8 & 2.2 & 2.9 \\
\hline Non-government (including public sector enterprises) & 16.4 & 16.0 & 15.5 & 16.4 & 16.1 & 16.4 & 17.2 & 17.9 \\
\hline Gross capital formation & 22.0 & 19.7 & 17.9 & 21.0 & 21.4 & 21.9 & 22.9 & 24.2 \\
\hline Government & 4.4 & 3.0 & 3.2 & 4.4 & 4.5 & 4.6 & 4.9 & 4.9 \\
\hline Non-government (including public sector enterprises) & 17.6 & 16.7 & 14.7 & 16.6 & 17.0 & 17.3 & 18.0 & 19.3 \\
\hline & \multicolumn{8}{|c|}{ (In billions of U.S. dollars, unless otherwise indicated) } \\
\hline \multicolumn{9}{|l|}{ Balance of payments } \\
\hline Current account balance & -13.9 & -9.3 & -6.7 & -7.7 & -8.0 & -7.8 & -7.9 & -8.4 \\
\hline Net capital flows $1 /$ & 1.3 & 1.0 & 1.0 & 1.2 & 1.3 & 1.4 & 1.5 & 1.6 \\
\hline Of which: foreign direct investment $2 /$ & 5.4 & 3.7 & 3.0 & 3.5 & 4.0 & 4.5 & 5.2 & 5.7 \\
\hline Gross official reserves & 8.6 & 9.1 & 13.5 & 17.8 & 18.4 & 17.4 & 16.0 & 16.0 \\
\hline In months of imports 3 / & 2.6 & 2.8 & 4.0 & 4.9 & 4.7 & 4.2 & 3.6 & 3.4 \\
\hline \multirow[t]{2}{*}{ External debt (in percent of GDP) } & 27.0 & 30.5 & 31.2 & 33.5 & 33.6 & 32.4 & 30.4 & 28.4 \\
\hline & \multicolumn{8}{|c|}{ (In percent of GDP) } \\
\hline \multicolumn{9}{|l|}{ Public finances } \\
\hline Revenue and grants & 14.9 & 14.3 & 14.9 & 15.4 & 15.9 & 16.2 & 16.6 & 16.9 \\
\hline Of which: tax revenue & 10.6 & 10.2 & 10.4 & 11.2 & 11.8 & 12.4 & 12.8 & 13.2 \\
\hline Expenditure (incl. stat. discr.), of which: & 22.2 & 19.3 & 19.5 & 19.3 & 19.0 & 19.0 & 19.3 & 18.9 \\
\hline Current & 18.1 & 16.0 & 16.2 & 14.9 & 14.5 & 14.4 & 14.4 & 14.1 \\
\hline Development (incl. net lending) & 4.1 & 3.1 & 3.4 & 4.4 & 4.5 & 4.6 & 4.9 & 4.9 \\
\hline Primary balance 4/ & -2.5 & -0.1 & -0.2 & -0.1 & 0.4 & 0.3 & 0.2 & 0.5 \\
\hline Overall fiscal balance 4/ & -7.3 & -5.0 & -4.6 & -3.8 & -3.1 & -2.8 & -2.7 & -2.0 \\
\hline Total public debt (including obligations to the IMF) & 59.3 & 58.8 & 60.9 & 61.5 & 59.3 & 56.2 & 52.5 & 48.3 \\
\hline \multicolumn{9}{|l|}{ Memorandum item } \\
\hline Real per capita consumption (percentage change) & 5.6 & 7.5 & 1.0 & 2.0 & 2.9 & 2.5 & 2.5 & 2.5 \\
\hline
\end{tabular}

Sources: Pakistani authorities for historical data; and Fund staff estimates and projections.

$1 /$ Difference between the overall balance and the current account balance.

2/ Including privatization.

$3 /$ In months of next year's imports of goods and services.

4/ Including grants. 
Table 6. Pakistan: Medium-Term Balance of Payments, 2007/08-2014/15

(In millions of U.S. dollars; unless otherwise indicated)

\begin{tabular}{|c|c|c|c|c|c|c|c|c|}
\hline & \multirow[b]{2}{*}{$2007 / 08$} & \multirow[b]{2}{*}{$2008 / 09$} & \multicolumn{6}{|c|}{ Projections } \\
\hline & & & $2009 / 10$ & $2010 / 11$ & $2011 / 12$ & $2012 / 13$ & $2013 / 14$ & $2014 / 15$ \\
\hline Current account & $-13,874$ & $-9,339$ & $-6,711$ & $-7,693$ & $-7,954$ & $-7,844$ & $-7,879$ & $-8,409$ \\
\hline Balance on goods & $-14,970$ & $-12,626$ & $-12,276$ & $-13,173$ & $-14,404$ & $-15,243$ & $-16,191$ & $-17,309$ \\
\hline Exports, f.o.b. & 20,427 & 19,121 & 18,964 & 19,609 & 20,533 & 21,946 & 23,535 & 25,178 \\
\hline Imports, f.o.b. & $-35,397$ & $-31,747$ & $-31,239$ & $-32,782$ & $-34,937$ & $-37,190$ & $-39,726$ & $-42,487$ \\
\hline Services (net) & $-6,457$ & $-3,473$ & $-2,960$ & $-3,815$ & $-4,271$ & $-4,564$ & $-4,889$ & $-5,274$ \\
\hline Services: credit & 3,589 & 4,014 & 4,588 & 4,277 & 4,479 & 4,795 & 5,136 & 5,496 \\
\hline Services: debit & $-10,046$ & $-7,487$ & $-7,548$ & $-8,092$ & $-8,750$ & $-9,359$ & $-10,025$ & $-10,770$ \\
\hline Income (net) & $-3,923$ & $-4,403$ & $-3,709$ & $-3,902$ & $-3,316$ & $-2,968$ & $-2,699$ & $-2,740$ \\
\hline Income: credit & 1,613 & 873 & 586 & 787 & 1,693 & 2,540 & 2,747 & 2,680 \\
\hline Income: debit & $-5,536$ & $-5,276$ & $-4,295$ & $-4,688$ & $-5,009$ & $-5,508$ & $-5,447$ & $-5,420$ \\
\hline Of which: interest payments & $-2,161$ & $-1,908$ & $-1,877$ & $-1,647$ & $-1,774$ & $-1,821$ & $-1,759$ & $-1,732$ \\
\hline Of which: income on direct investment & $-3,375$ & $-3,368$ & $-2,418$ & $-3,042$ & $-3,234$ & $-3,687$ & $-3,950$ & $-4,285$ \\
\hline Balance on goods, services, and income & $-25,350$ & $-20,502$ & $-18,945$ & $-20,889$ & $-21,991$ & $-22,775$ & $-23,779$ & $-25,323$ \\
\hline Current transfers (net) & 11,476 & 11,163 & 12,234 & 13,196 & 14,036 & 14,932 & 15,900 & 16,915 \\
\hline Current transfers: credit, of which:1/ & 11,618 & 11,265 & 12,319 & 13,304 & 14,144 & 15,040 & 16,008 & 17,023 \\
\hline Official & 484 & 219 & 179 & 375 & 375 & 375 & 390 & 390 \\
\hline Workers' remittances & 6,451 & 7,811 & 8,375 & 8,919 & 9,499 & 10,117 & 10,774 & 11,474 \\
\hline Other private transfers & 4,683 & 3,235 & 3,765 & 4,010 & 4,270 & 4,548 & 4,844 & 5,158 \\
\hline Current transfers: debit & -142 & -102 & -85 & -108 & -108 & -108 & -108 & -108 \\
\hline Capital account & 121 & 474 & 1,288 & 385 & 208 & 214 & 220 & 226 \\
\hline Capital transfers: credit & 128 & 479 & 1,291 & 388 & 211 & 217 & 223 & 229 \\
\hline Of which: official capital grants & 111 & 427 & 705 & 279 & 100 & 100 & 100 & 100 \\
\hline Of which: Tokyo pledges & $\ldots$ & $\ldots$ & 325 & 822 & 548 & $\ldots$ & 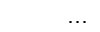 & \\
\hline Capital transfers: debit & -7 & -5 & -3 & -3 & -3 & -3 & -3 & -3 \\
\hline Financial account & 8,131 & 5,720 & 6,792 & 8,750 & 9,821 & 9,958 & 10,999 & 11,291 \\
\hline Direct investment abroad & -75 & -25 & -40 & -50 & -10 & -9 & -8 & -7 \\
\hline Direct investment in Pakistan & 5,410 & 3,720 & 2,954 & 3,500 & 4,000 & 4,500 & 5,200 & 5,700 \\
\hline Of which: privatization receipts & 133 & 0 & 800 & 300 & 300 & 300 & 200 & 200 \\
\hline Portfolio investment (net), of which: & 32 & $-1,096$ & -84 & 478 & 1,300 & 1,500 & 1,700 & 2,100 \\
\hline Eurobond/GDR & -25 & -544 & -622 & -22 & 500 & 500 & 500 & 500 \\
\hline Other investment assets & 32 & 560 & -646 & -448 & -460 & -473 & -486 & -500 \\
\hline General government & 5 & 8 & -8 & -8 & -8 & -8 & -8 & -8 \\
\hline Banks & 525 & 346 & -200 & -200 & -200 & -200 & -200 & -200 \\
\hline Other sectors & -498 & 206 & -76 & -240 & -252 & -265 & -278 & -292 \\
\hline Other investment liabilities & 2,732 & 2,561 & 4,608 & 5,270 & 4,991 & 4,439 & 4,593 & 3,998 \\
\hline Monetary authorities & 490 & -1 & 1,250 & 0 & 0 & 0 & 0 & 0 \\
\hline General government, of which: & 1,761 & 1,969 & 2,141 & 4,711 & 4,515 & 4,089 & 3,943 & 2,681 \\
\hline Disbursements $1 /$ & 3,054 & 3,642 & 4,787 & 6,575 & 6,275 & 5,875 & 5,860 & 4,700 \\
\hline Of which: Tokyo pledges & $\ldots$ & $\ldots$ & 1,132 & $\ldots$ & $\ldots$ & $\ldots$ & $\ldots$ & \\
\hline Amortization & $-1,272$ & $-2,246$ & $-2,646$ & $-1,864$ & $-1,760$ & $-1,786$ & $-1,917$ & $-2,019$ \\
\hline Banks & 66 & 291 & 100 & 100 & 100 & 100 & 100 & 100 \\
\hline Other sectors & 415 & 302 & 1,116 & 459 & 376 & 250 & 550 & 1,216 \\
\hline Net errors and omissions & -166 & -89 & -36 & 0 & 0 & 0 & 0 & 0 \\
\hline Reserves and related items & 5,788 & 3,234 & $-1,333$ & $-1,442$ & $-2,075$ & $-2,328$ & $-3,339$ & $-3,108$ \\
\hline Reserve assets, of which: & 5,961 & -457 & $-4,574$ & $-4,664$ & -950 & 580 & 950 & -500 \\
\hline Foreign exchange (State Bank of Pakistan) & 5,711 & -523 & $-4,427$ & $-4,284$ & -550 & 1,000 & 1,400 & 0 \\
\hline Foreign exchange (deposit money banks) & 250 & 66 & -147 & -380 & -400 & -420 & -450 & -500 \\
\hline Use of Fund credit and loans & -173 & 3,691 & 3,341 & 3,222 & $-1,125$ & $-2,908$ & $-4,289$ & $-2,608$ \\
\hline \multicolumn{9}{|l|}{ Memorandum items: } \\
\hline Current account (in percent of GDP; including official transfers) & -8.4 & -5.6 & -3.8 & -4.0 & -3.9 & -3.6 & -3.4 & -3.4 \\
\hline Current account (in percent of GDP; excluding fuel imports) & -2.1 & 0.4 & 2.1 & 2.2 & 2.4 & 2.8 & 3.0 & 3.0 \\
\hline Exports f.o.b. (growth rate, in percent) & 18.2 & -6.4 & -0.8 & 3.4 & 4.7 & 6.9 & 7.2 & 7.0 \\
\hline Imports f.o.b. (growth rate, in percent) & 31.2 & -10.3 & -1.6 & 4.9 & 6.6 & 6.4 & 6.8 & 7.0 \\
\hline Imports f.o.b. (growth rate, in percent, excluding oil) & 26.8 & -12.2 & -3.7 & 0.7 & 5.5 & 6.2 & 6.7 & 7.0 \\
\hline Oil imports (in million US\$, cif) & 10,495 & 10,031 & 10,389 & 11,881 & 12,925 & 13,830 & 14,817 & 15,834 \\
\hline Crude oil price (\$/bbl) & 87.0 & 68.5 & 73.6 & 84.2 & 88.9 & 91.3 & 93.3 & 94.4 \\
\hline External debt (in millions of U.S. dollars) & 44,468 & 50,759 & 55,774 & 64,050 & 68,233 & 70,089 & 70,295 & 70,789 \\
\hline Gross financing needs (in millions of U.S. dollars) 2/ & 15,228 & 12,295 & 10,211 & 9,836 & 10,840 & 12,537 & 14,086 & 13,035 \\
\hline End-period gross official reserves (millions of U.S. dollars) 3/ & 8,591 & 9,114 & 13,541 & 17,825 & 18,375 & 17,375 & 15,975 & 15,975 \\
\hline (In months of next year's imports of goods and services) & 2.6 & 2.8 & 4.0 & 4.9 & 4.7 & 4.2 & 3.6 & 3.4 \\
\hline GDP (in millions of U.S. dollars) & 164,557 & 166,515 & 178,922 & 191,062 & 202,909 & 216,036 & 231,274 & 248,815 \\
\hline
\end{tabular}

Sources: Pakistani authorities; and Fund staff estimates and projections.

1/ For 2010/11-2013/14, includes total U.S. Kerry-Lugar support of $\$ 7.5$ billon of which 15 percent assumed to be current transfers, and 85 percent as project support. 2/ Defined as current account deficit, plus amortization on medium- and long-term debt, plus short-term debt at end of previous period.

3/ Excluding foreign currency deposits held with the State Bank of Pakistan (cash reserve requirements) and gold. 
Table 7. Pakistan: Medium-Term Fiscal Framework, 2007/08-2014/15

(In percent of GDP; unless otherwise indicated)

\begin{tabular}{|c|c|c|c|c|c|c|c|c|}
\hline & \multirow[b]{2}{*}{ 2007/08 } & \multirow[b]{2}{*}{ 2008/09 } & \multicolumn{6}{|c|}{ Projections } \\
\hline & & & $2009 / 10$ & $2010 / 11$ & $2011 / 12$ & $2012 / 13$ & $2013 / 14$ & $2014 / 15$ \\
\hline Revenue and grants & 14.9 & 14.3 & 14.9 & 15.4 & 15.9 & 16.2 & 16.6 & 16.9 \\
\hline Tax revenue & 10.6 & 10.2 & 10.4 & 11.2 & 11.8 & 12.4 & 12.8 & 13.2 \\
\hline Of which: Federal Board of Revenue & 9.8 & 8.8 & 9.2 & 10.0 & 10.7 & 11.3 & 11.7 & 12.2 \\
\hline Nontax revenue & 4.0 & 4.0 & 4.0 & 3.9 & 3.8 & 3.6 & 3.6 & 3.6 \\
\hline Grants & 0.3 & 0.2 & 0.5 & 0.3 & 0.2 & 0.2 & 0.2 & 0.2 \\
\hline Expenditure & 22.2 & 19.1 & 19.5 & 19.3 & 19.0 & 19.0 & 19.3 & 18.9 \\
\hline Current expenditure & 18.1 & 16.0 & 16.2 & 14.9 & 14.5 & 14.4 & 14.4 & 14.1 \\
\hline Interest & 4.8 & 4.9 & 4.4 & 3.8 & 3.5 & 3.1 & 2.8 & 2.5 \\
\hline Other federal & 9.1 & 6.9 & 8.1 & 6.7 & 6.5 & 6.7 & 6.9 & 6.9 \\
\hline Provincial & 4.3 & 4.2 & 3.7 & 4.4 & 4.5 & 4.6 & 4.6 & 4.6 \\
\hline Development expenditure & 4.4 & 3.0 & 3.2 & 4.4 & 4.5 & 4.6 & 4.9 & 4.9 \\
\hline Net-lending & -0.3 & 0.1 & 0.1 & 0.0 & 0.0 & 0.0 & 0.0 & 0.0 \\
\hline Statistical discrepancy & 0.0 & 0.3 & 0.0 & 0.0 & 0.0 & 0.0 & 0.0 & 0.0 \\
\hline \multicolumn{9}{|l|}{ Overall balance } \\
\hline Excluding grants & -7.6 & -5.2 & -5.1 & -4.2 & -3.3 & -3.0 & -2.9 & -2.2 \\
\hline Including grants & -7.3 & -5.0 & -4.6 & -3.8 & -3.1 & -2.8 & -2.7 & -2.0 \\
\hline Financing & 7.3 & 5.0 & 4.6 & 3.8 & 3.1 & 2.8 & 2.7 & 2.0 \\
\hline External & 1.2 & 0.5 & 1.8 & 2.5 & 2.5 & 2.1 & 1.9 & 2.1 \\
\hline Of which: privatization receipts & 0.0 & 0.0 & 0.4 & 0.2 & 0.1 & 0.1 & 0.1 & 0.1 \\
\hline Domestic & 6.1 & 4.5 & 2.7 & 1.4 & 0.6 & 0.7 & 0.7 & -0.1 \\
\hline \multicolumn{9}{|l|}{ Memorandum items: } \\
\hline \multicolumn{9}{|l|}{ Primary balance } \\
\hline Excluding grants & -2.8 & -0.3 & -0.7 & -0.4 & 0.1 & 0.1 & 0.0 & 0.3 \\
\hline Including grants & -2.5 & -0.1 & -0.2 & -0.1 & 0.4 & 0.3 & 0.2 & 0.5 \\
\hline Interest payments/revenue (ratio) & 32.7 & 34.5 & 30.4 & 24.9 & 22.2 & 19.6 & 17.3 & 15.1 \\
\hline Public debt $1 /$ & 58.4 & 55.6 & 56.4 & 55.3 & 54.1 & 52.7 & 51.2 & 48.1 \\
\hline External & 26.7 & 26.2 & 25.6 & 25.9 & 26.6 & 26.9 & 26.8 & 26.0 \\
\hline Domestic & 31.8 & 29.4 & 30.8 & 29.4 & 27.5 & 25.9 & 24.3 & 22.1 \\
\hline Public debt (including obligations to the IMF) & 59.3 & 58.8 & 60.9 & 61.5 & 59.3 & 56.2 & 52.5 & 48.3 \\
\hline Nominal GDP (billions of Pakistani rupees) & 10,284 & 13,095 & 15,039 & 16,814 & 18,712 & 20,827 & 23,291 & 26,169 \\
\hline
\end{tabular}

Sources: Pakistani authorities for historical data; and Fund staff for estimates and projections.

1/ Excludes obligations to the IMF except budget financing, military debt, commercial loans, and short-term debt. 
Table 8. Pakistan: External Debt, 2007/08-2014/15

(In millions of U.S. dollars, outstanding at end of each fiscal year)

\begin{tabular}{|c|c|c|c|c|c|c|c|c|}
\hline & \multirow[b]{2}{*}{$2007 / 08$} & \multirow[b]{2}{*}{$2008 / 09$} & \multicolumn{6}{|c|}{ Projections } \\
\hline & & & $2009 / 10$ & $2010 / 11$ & $2011 / 12$ & $2012 / 13$ & $2013 / 14$ & $2014 / 15$ \\
\hline Total external debt & 44,469 & 50,759 & 55,774 & 64,040 & 68,223 & 70,079 & 70,285 & 70,779 \\
\hline Public and publicly guaranteed debt (incl. IMF) & 41,582 & 47,259 & 51,980 & 59,881 & 63,771 & 65,452 & 65,606 & 66,180 \\
\hline Public and publicly guaranteed debt (excl. IMF) & 40,245 & 42,111 & 43,648 & 48,337 & 53,352 & 57,942 & 62,384 & 65,566 \\
\hline Medium- and long-term debt & 39,432 & 41,459 & 43,392 & 48,137 & 53,152 & 57,742 & 62,184 & 65,166 \\
\hline Multilateral creditors & 21,536 & 23,001 & 24,618 & 26,191 & 27,686 & 29,234 & 30,711 & 31,813 \\
\hline ADB & 9,401 & 9,035 & 10,041 & 11,219 & 12,325 & 13,256 & 14,132 & 14,960 \\
\hline World Bank & 11,483 & 12,017 & 12,639 & 13,084 & 13,528 & 14,204 & 14,871 & 15,221 \\
\hline Other & 651 & 1,949 & 1,938 & 1,887 & 1,833 & 1,774 & 1,707 & 1,633 \\
\hline Bilateral creditors & 15,181 & 15,947 & 17,246 & 20,441 & 23,460 & 26,001 & 28,467 & 29,846 \\
\hline Paris Club & 13,936 & 13,998 & 13,868 & 13,729 & 13,578 & 13,409 & 13,219 & 13,004 \\
\hline Non-paris club & 1,245 & 1,949 & 3,378 & 6,711 & 9,882 & 12,592 & 15,249 & 16,842 \\
\hline Bonds & 2,650 & 2,150 & 1,528 & 1,506 & 2,006 & 2,506 & 3,006 & 3,506 \\
\hline Of which: Euro bond & 2,650 & 2,150 & 1,528 & 1,506 & 2,006 & 2,506 & 3,006 & 3,506 \\
\hline Commercial banks and others & 65 & 361 & 0 & 0 & 0 & 0 & 0 & 0 \\
\hline Short-term debt & 813 & 652 & 256 & 200 & 200 & 200 & 200 & 400 \\
\hline Of which: IDB & 713 & 652 & 256 & 200 & 200 & 200 & 200 & 400 \\
\hline IMF & 1,337 & 5,148 & 8,332 & 11,543 & 10,418 & 7,511 & 3,222 & 614 \\
\hline Private sector debt & 2,887 & 3,500 & 3,795 & 4,159 & 4,453 & 4,626 & 4,679 & 4,599 \\
\hline \multicolumn{9}{|l|}{ Of which: } \\
\hline Multilateral creditors & 322 & $\ldots$ & $\ldots$ & $\ldots$ & $\ldots$ & $\cdots$ & $\ldots$ & ... \\
\hline Paris Club creditors & 1,274 & $\ldots$ & $\ldots$ & $\ldots$ & $\ldots$ & $\ldots$ & $\ldots$ & $\ldots$ \\
\hline Non-paris club creditors & 9 & $\ldots$ & $\ldots$ & $\ldots$ & $\ldots$ & $\ldots$ & $\ldots$ & $\ldots$ \\
\hline Other & 1,007 & $\ldots$ & $\ldots$ & $\ldots$ & $\ldots$ & $\ldots$ & $\ldots$ & $\ldots$ \\
\hline
\end{tabular}

Sources: Pakistani authorities; and Fund staff estimates and projections. 
Table 9. Pakistan: Gross Financing Requirements and Sources, 2007/08-20010/11 (In millions of U.S. dollars; unless otherwise specified)

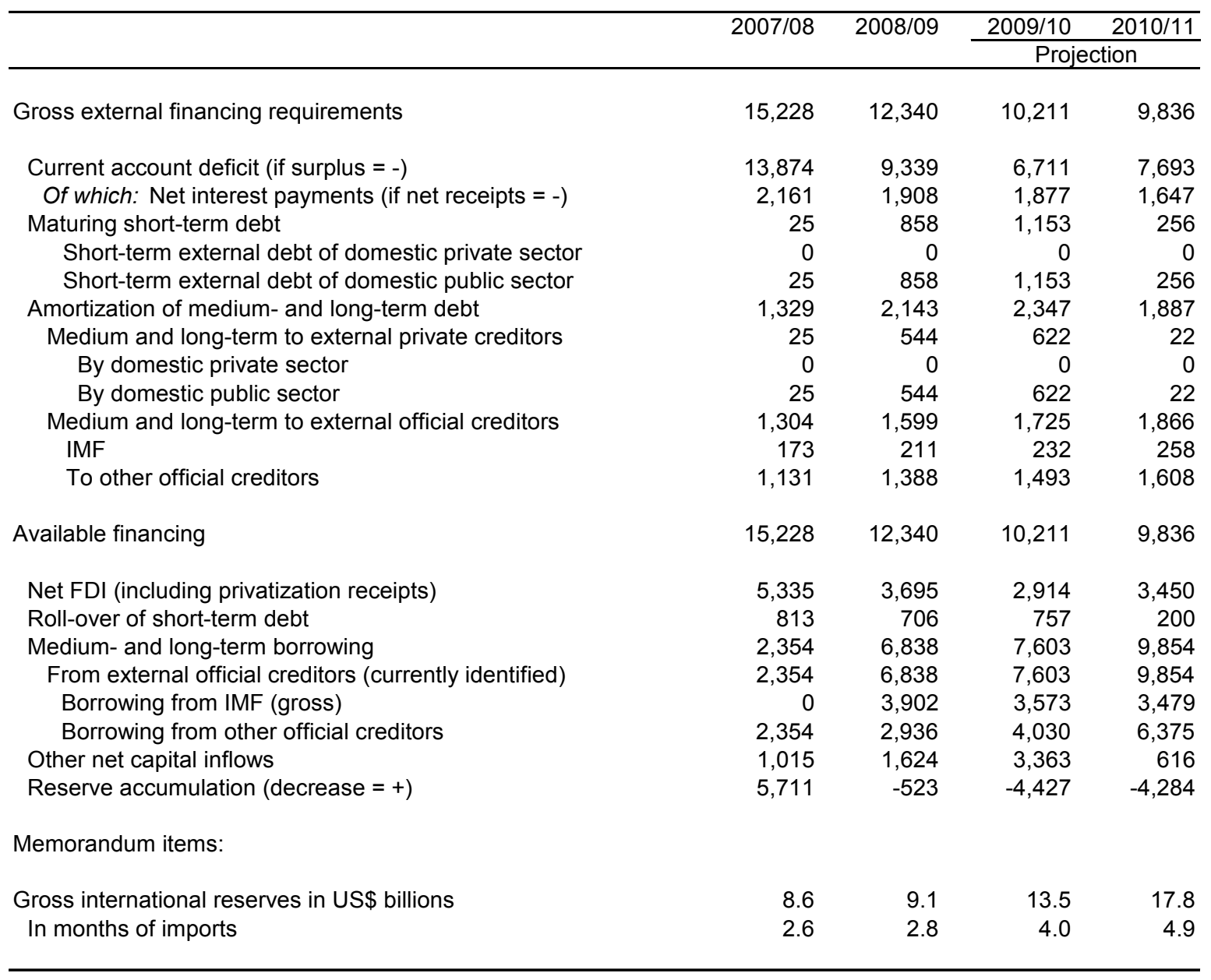

Sources: State Bank of Pakistan, and Fund staff estimates and projections. 
Table 10. Pakistan: Indicators of Fund Credit, 2008/09-2015/16

(In millions of SDR unless otherwise specified)

\begin{tabular}{|c|c|c|c|c|c|c|c|c|}
\hline & \multirow[b]{2}{*}{$2008 / 09$} & \multicolumn{7}{|c|}{ Projections } \\
\hline & & $2009 / 10$ & $2010 / 11$ & $2011 / 12$ & $2012 / 13$ & $2013 / 14$ & $2014 / 15$ & $2015 / 16$ \\
\hline & \multicolumn{8}{|c|}{ (Projected Debt Service to the Fund based on Existing and Prospective Drawings) } \\
\hline \multicolumn{9}{|l|}{ PRGF } \\
\hline Principal $1 /$ & 137.7 & 155.0 & 172.3 & 163.6 & 120.6 & 51.7 & 17.2 & 0.0 \\
\hline \multicolumn{8}{|l|}{ Stand-By Arrangements } & 0.0 \\
\hline Principal & 0.0 & 0.0 & 0.0 & 587.9 & $1,821.9$ & $2,813.7$ & $1,725.1$ & 287.5 \\
\hline \multirow[t]{2}{*}{ Interest and charges } & 42.9 & 59.3 & 151.8 & 171.5 & 159.6 & 75.1 & 18.0 & 2.1 \\
\hline & \multicolumn{8}{|c|}{ (Projected Level of Credit Outstanding based on Existing and Prospective Drawings) } \\
\hline Total & $3,316.4$ & $5,461.5$ & $7,589.3$ & $6,837.7$ & $4,895.2$ & $2,029.8$ & 287.5 & 0.0 \\
\hline PRGF Arrangements & 680.5 & 525.5 & 353.2 & 189.5 & 68.9 & 17.2 & 0.0 & 0.0 \\
\hline \multirow[t]{2}{*}{ Stand-by Arrangements } & $2,635.9$ & $4,936.0$ & $7,236.1$ & $6,648.2$ & $4,826.3$ & $2,012.6$ & 287.5 & 0.0 \\
\hline & \multicolumn{8}{|c|}{ (Projected Debt Service to the Fund based on Existing and Prospective Drawings) } \\
\hline Total $1 /$ & 184.6 & 216.1 & 324.1 & 923.4 & $2,102.4$ & $2,940.6$ & $1,760.3$ & 289.6 \\
\hline \multicolumn{9}{|l|}{ Of which: } \\
\hline Principal & 137.7 & 155.0 & 172.3 & 751.6 & $1,942.5$ & $2,865.4$ & $1,742.3$ & 287.5 \\
\hline Interest and charges & 46.9 & 61.0 & 151.8 & 171.8 & 159.9 & 75.2 & 18.0 & 2.1 \\
\hline In percent of exports of goods and non-factor services & 0.0 & 0.0 & 0.0 & 0.0 & 0.0 & 0.0 & 0.0 & 0.0 \\
\hline In percent of GDP & 0.0 & 0.0 & 0.0 & 0.0 & 0.0 & 0.0 & 0.0 & 0.0 \\
\hline In percent of end-period gross international reserves & 0.0 & 0.0 & 0.0 & 0.0 & 0.0 & 0.0 & 0.0 & 0.0 \\
\hline \multicolumn{9}{|l|}{ Memorandum items } \\
\hline Exports of goods and NFS (in millions of U.S. dollars) & 23,135 & 23,552 & 23,886 & 25,012 & 26,741 & 28,671 & 30,674 & 32,862 \\
\hline Quota & 1,034 & & & & & & & \\
\hline GDP (in millions of U.S. dollars) & 166,515 & 178,922 & 191,062 & 202,909 & 216,036 & 231,274 & 248,815 & 267,687 \\
\hline Fund credit outstanding (in percent of GDP) & 0.0 & 0.0 & 0.0 & 0.0 & 0.0 & 0.0 & 0.0 & 0.0 \\
\hline Of which: SBA & 0.0 & 0.0 & 0.0 & 0.0 & 0.0 & 0.0 & 0.0 & 0.0 \\
\hline Gross international reserves (in millions of U.S. dollars) & 9,114 & 13,541 & 17,825 & 18,375 & 17,375 & 15,975 & 15,975 & 18,075 \\
\hline
\end{tabular}

Source: Fund staff projections.

1/ For 2008/09, debt service includes payments related to EFF. 
Table 11. Pakistan: Access and Phasing under the Stand-By Arrangement, 2008-10

\begin{tabular}{|c|c|c|c|c|c|c|}
\hline \multirow[t]{2}{*}{ Review } & \multirow[t]{2}{*}{ Availability Date } & \multirow[t]{2}{*}{ Action } & \multicolumn{2}{|c|}{ Purchase } & \multicolumn{2}{|c|}{$\begin{array}{l}\text { Of which: for budget } \\
\text { financing }\end{array}$} \\
\hline & & & $\begin{array}{l}\text { In millions of } \\
\text { SDRs }\end{array}$ & $\begin{array}{c}\text { In percent } \\
\text { of quota }\end{array}$ & $\begin{array}{c}\text { In millions of } \\
\text { SDRs }\end{array}$ & $\begin{array}{c}\text { In percent of } \\
\text { quota }\end{array}$ \\
\hline \multicolumn{7}{|c|}{ Approved phasing } \\
\hline & November 24, 2008 & Board approval of SBA & $2,067.400$ & 200.00 & $\ldots$ & $\ldots$ \\
\hline First Review & March 15, 2009 1/ & Observance of end-December 2008 performance criteria, completion of first review & 568.535 & 55.00 & $\ldots$ & $\ldots$ \\
\hline Second Review & June $15,20092 /$ & Observance of end-March 2009 performance criteria, completion of second review & 766.700 & 74.17 & 475.60 & 46.01 \\
\hline Third Review & November 30, 2009 3/ & Observance of end-September 2009 performance criteria, completion of third review & 766.700 & 74.17 & 237.75 & 23.00 \\
\hline Fourth Review & February 28, $20104 /$ & Observance of end-December 2009 performance criteria, completion of fourth review & 766.700 & 74.17 & 237.75 & 23.00 \\
\hline Fifth Review & May 31, 2010 & Observance of end-March 2010 performance criteria, completion of fifth review & 766.700 & 74.17 & $\ldots$ & $\ldots$ \\
\hline Sixth Review & August 31,2010 & Observance of end-June 2010 performance criteria, completion of sixth review & 766.700 & 74.17 & $\ldots$ & $\ldots$ \\
\hline Seventh Review & November 30, 2010 & Observance of end-September 2010 performance criteria, completion of seventh review & 766.465 & 74.15 & $\ldots$ & $\ldots$ \\
\hline Total & & & $7,235.900$ & 700.000 & 951.10 & 92.01 \\
\hline \multicolumn{7}{|c|}{ Proposed rephasing } \\
\hline Fifth Review & August 15, 2010 & Observance of end-June 2010 performance criteria, completion of fifth review & $1,150.050$ & 111.26 & $\ldots$ & $\ldots$ \\
\hline Sixth Review & November 15, 2010 & Observance of end-September 2010 performance criteria, completion of sixth review & $1,149.815$ & 111.23 & $\ldots$ & $\ldots$ \\
\hline Total to be repha & & & $2,299.865$ & 222.49 & $\ldots$ & $\ldots$ \\
\hline
\end{tabular}

Source: Fund staff.

1/ The first review was completed on March 30 and the second purchase was made on April 1.

2/ The second review was completed on August 7 and the third purchase was made on August 11; the availability of the third purchase became conditional on the observance of end-June 2009 PCs. 3/ The third review was completed on December 23 and the fourth purchase was made on December 28.

4/ The fourth review was delayed beyond end-March 2010 making the availability of the fifth purchase conditional on the observance of end-March 2010 PCs. 
Table 12. Pakistan: Selected Vulnerability Indicators, 2007/08-2014/15

\begin{tabular}{|c|c|c|c|c|c|c|c|c|}
\hline & \multirow[t]{2}{*}{$2007 / 08$} & \multirow[t]{2}{*}{$2008 / 09$} & $2009 / 10$ & $2010 / 11$ & $2011 / 12$ & $2012 / 13$ & $2013 / 14$ & $2014 / 15$ \\
\hline & & & & & \multicolumn{2}{|c|}{ Projection } & & \\
\hline \multicolumn{9}{|l|}{ Key economic and market indicators } \\
\hline Real GDP growth (factor cost, in percent) & 4.1 & 2.0 & 3.0 & 4.0 & 4.5 & 5.0 & 5.5 & 6.0 \\
\hline $\mathrm{CPI}$ inflation (period average, in percent) & 12.0 & 20.8 & 11.5 & 7.5 & 6.5 & 6.0 & 6.0 & 6.0 \\
\hline Short-term (ST) interest rate (in percent) & 11.5 & $\ldots$ & $\ldots$ & $\ldots$ & $\ldots$ & $\ldots$ & $\ldots$ & $\ldots$ \\
\hline \multicolumn{9}{|l|}{ Emerging market bond index (EMBI) secondary market } \\
\hline spread (basis points, end of period) $8 /$ & 687 & 1,039 & 490 & $\ldots$ & $\ldots$ & $\ldots$ & $\ldots$ & $\ldots$ \\
\hline Exchange rate PRs/US\$ (end of period) 8/ & 68.2 & 81.4 & 84.1 & $\ldots$ & $\ldots$ & $\ldots$ & $\ldots$ & $\cdots$ \\
\hline \multicolumn{9}{|l|}{ External sector } \\
\hline Exchange rate regime & \multicolumn{8}{|c|}{ Floating since January 2008} \\
\hline Current account balance (percent of GDP) & -8.4 & -5.6 & -3.8 & -4.0 & -3.9 & -3.6 & -3.4 & -3.4 \\
\hline Net FDI inflows (percent of GDP) & 3.2 & 2.2 & 1.6 & 1.8 & 2.0 & 2.1 & 2.2 & 2.3 \\
\hline Exports (percentage change of U.S. dollar value; GNFS) & 12.1 & -3.7 & 1.8 & 1.4 & 4.7 & 6.9 & 7.2 & 7.0 \\
\hline Real effective exchange rate $(2000=100$, annual average $)$ & 94.8 & $\ldots$ & $\ldots$ & $\ldots$ & $\ldots$ & $\ldots$ & $\ldots$ & $\ldots$ \\
\hline Gross international reserves $(G I R)$ in billions of U.S. dollars & 8.6 & 9.1 & 13.5 & 17.8 & 18.4 & 17.4 & 16.0 & 16.0 \\
\hline GIR in percent of ST debt at remaining maturity (RM) 1/ & 436.3 & 366.8 & 642.3 & 974.2 & $1,043.9$ & 973.1 & 833.2 & 720.0 \\
\hline GIR in percent of ST debt at RM and banks' foreign exchange (FX) deposits 1/ & 147.3 & 153.8 & 227.8 & 296.5 & 293.8 & 262.7 & 224.0 & 202.9 \\
\hline Total gross external debt (ED) in percent of GDP, of which: & 27.0 & 30.5 & 31.2 & 33.5 & 33.6 & 32.4 & 30.4 & 28.4 \\
\hline ST external debt (original maturity, in percent of total ED) & 1.8 & 1.3 & 0.5 & 0.3 & 0.3 & 0.3 & 0.3 & 0.6 \\
\hline ED of domestic private sector (in percent of total ED) & 6.5 & 6.9 & 6.8 & 6.5 & 6.5 & 6.6 & 6.7 & 6.5 \\
\hline ED to foreign official sector (in percent of total ED) & 93.5 & 93.1 & 93.2 & 93.5 & 93.5 & 93.4 & 93.3 & 93.5 \\
\hline Total gross external debt in percent of exports & 185.2 & 219.4 & 236.7 & 268.1 & 272.7 & 262.0 & 245.1 & 230.7 \\
\hline Gross external financing requirement (in billions of U.S. dollars) $2 /$ & 15.2 & 12.3 & 10.2 & 9.8 & 10.8 & 12.5 & 14.1 & 13.0 \\
\hline Public sector $3 /$ & \multicolumn{8}{|c|}{ (In percent of GDP) } \\
\hline Overall balance (including grants) & -7.3 & -5.0 & -4.6 & -3.8 & -3.1 & -2.8 & -2.7 & -2.0 \\
\hline Primary balance (including grants) & -2.5 & -0.1 & -0.2 & -0.1 & 0.4 & 0.3 & 0.2 & 0.5 \\
\hline Debt-stabilizing primary balance 4 / & -1.4 & -3.7 & -1.4 & -1.5 & -1.4 & -1.6 & -1.9 & 0.0 \\
\hline Gross PS financing requirement $5 /$ & 24.7 & 22.0 & 21.8 & 19.8 & 18.0 & 16.9 & 15.8 & 14.1 \\
\hline Public sector gross debt & 58.4 & 55.6 & 56.4 & 55.3 & 54.1 & 52.7 & 51.2 & 48.1 \\
\hline Public sector net debt $6 /$ & 53.8 & 51.0 & 51.8 & 50.7 & 49.5 & 48.1 & 46.5 & 43.4 \\
\hline \multicolumn{9}{|l|}{ Financial sector $7 l$} \\
\hline Capital adequacy ratio (in percent) 9/ & 12.1 & 13.5 & 14.1 & $\ldots$ & $\ldots$ & $\ldots$ & $\ldots$ & $\ldots$ \\
\hline Nonperforming loans (NPLs) in percent of total loans 9/ & 7.7 & 11.5 & 12.2 & $\ldots$ & $\ldots$ & $\ldots$ & $\ldots$ & $\ldots$ \\
\hline Provisions in percent of NPLs 9/ & 84.0 & 70.2 & 71.0 & $\ldots$ & $\ldots$ & $\ldots$ & $\ldots$ & $\ldots$ \\
\hline Return on average assets (after tax, in percent) 9/ & 1.7 & 1.0 & 0.9 & $\ldots$ & $\ldots$ & $\ldots$ & $\ldots$ & $\ldots$ \\
\hline Return on equity (after tax, in percent) $9 /$ & 16.7 & 9.7 & 8.6 & $\ldots$ & $\ldots$ & $\ldots$ & $\ldots$ & $\ldots$ \\
\hline FX deposits held by residents (in percent of total deposits) & 7.1 & 7.0 & 7.3 & $\ldots$ & $\ldots$ & $\ldots$ & $\ldots$ & $\ldots$ \\
\hline Government debt held by FS (percent of total FS assets) & 31.4 & 38.9 & 36.3 & $\ldots$ & $\cdots$ & $\ldots$ & $\ldots$ & $\ldots$ \\
\hline Credit to private sector (percent change) & 16.4 & 0.7 & 11.6 & $\ldots$ & $\ldots$ & $\ldots$ & $\ldots$ & $\ldots$ \\
\hline \multicolumn{9}{|l|}{ Memorandum item: } \\
\hline Nominal GDP (in billions of U.S. dollars) & 164.6 & 166.5 & 178.9 & 191.1 & 202.9 & 216.0 & 231.3 & 248.8 \\
\hline
\end{tabular}

Sources: Pakistani authorities; and Fund staff estimates and projections

$1 /$ Debt at remaining maturity is defined as maturing short-, medium-, and long-term external official debt.

2/ Current account deficit plus amortization of external debt.

3/ Public sector covers general (consolidated) government.

4/ Based on the end of period debt stock in year $\mathrm{t}-1$, and the baseline assumptions for the relevant variables (i.e., growth, interest rates,

inflation, exchange rates) in year $t$.

$5 /$ Overall balance plus debt amortization.

6/ Net debt is defined as gross debt minus government deposits with the banking system.

7/ Financial sector includes all commercial and specialized banks; for government debt also includes nonbanks, but excludes State Bank of Pakistan.

$8 / 2009 / 10$ is end-March data.

/ 2009/10 is end-December data. 
Table 13. Pakistan: Financial Soundness Indicators for the Banking System

(December 2006-December 2009)

\begin{tabular}{|c|c|c|c|c|c|c|c|}
\hline & $\begin{array}{l}\text { Dec. } \\
2006\end{array}$ & $\begin{array}{r}\text { Dec. } \\
2007 \text { 1/ }\end{array}$ & $\begin{array}{r}\text { Dec. } \\
20081 /\end{array}$ & $\begin{array}{l}\text { Mar. } \\
2009\end{array}$ & $\begin{array}{l}\text { Jun. } \\
2009\end{array}$ & $\begin{array}{l}\text { Sep. } \\
2009\end{array}$ & $\begin{array}{l}\text { Dec. } \\
2009\end{array}$ \\
\hline \multicolumn{8}{|l|}{ Capital adequacy } \\
\hline Regulatory capital to risk-weighted assets & 12.7 & 12.3 & 12.3 & 12.9 & 13.5 & 14.3 & 14.1 \\
\hline Tier I capital to risk-weighted assets & 10.0 & 10.0 & 10.2 & 10.8 & 11.3 & 11.9 & 11.6 \\
\hline Capital to total assets & 9.4 & 10.5 & 10.0 & 10.3 & 10.2 & 10.5 & 10.1 \\
\hline \multicolumn{8}{|l|}{ Asset composition and quality } \\
\hline Nonperforming loans (NPLs) to gross loans & 6.9 & 7.6 & 10.5 & 11.5 & 11.5 & 12.4 & 12.2 \\
\hline Provisions to NPLs & 77.8 & 86.1 & 69.6 & 69.2 & 70.2 & 69.7 & 71.0 \\
\hline NPLs net of provisions to capital & 9.7 & 5.6 & 19.4 & 19.6 & 19.0 & 19.9 & 18.9 \\
\hline \multicolumn{8}{|l|}{ Earnings and profitability } \\
\hline Return on assets (after tax) & 2.1 & 1.5 & 0.8 & 1.1 & 1.0 & 0.9 & 0.9 \\
\hline Return on equity (after tax) & 23.8 & 15.4 & 7.8 & 10.7 & 9.7 & 9.0 & 8.6 \\
\hline Net interest income to gross income & 70.9 & 68.2 & 70.4 & 75.8 & 73.0 & 73.1 & 72.3 \\
\hline Noninterest expenses to gross income & 40.3 & 43.2 & 50.3 & 49.9 & 50.1 & 50.1 & 51.6 \\
\hline \multicolumn{8}{|l|}{ Liquidity } \\
\hline Liquid assets to total assets & 31.9 & 33.6 & 28.6 & 30.5 & 31.2 & 31.4 & 32.3 \\
\hline Liquid assets to total deposits & 42.7 & 45.1 & 38.2 & 41.5 & 41.7 & 42.7 & 44.1 \\
\hline
\end{tabular}

Source: State Bank of Pakistan.

1/ Data for 2007 and 2008 have been restated on the basis of annual audits. 
Appendix. Pakistan: Debt Sustainability Analysis

Figure 1. Pakistan: External Debt Sustainability-Bound Tests 1/

(External debt in percent of GDP)
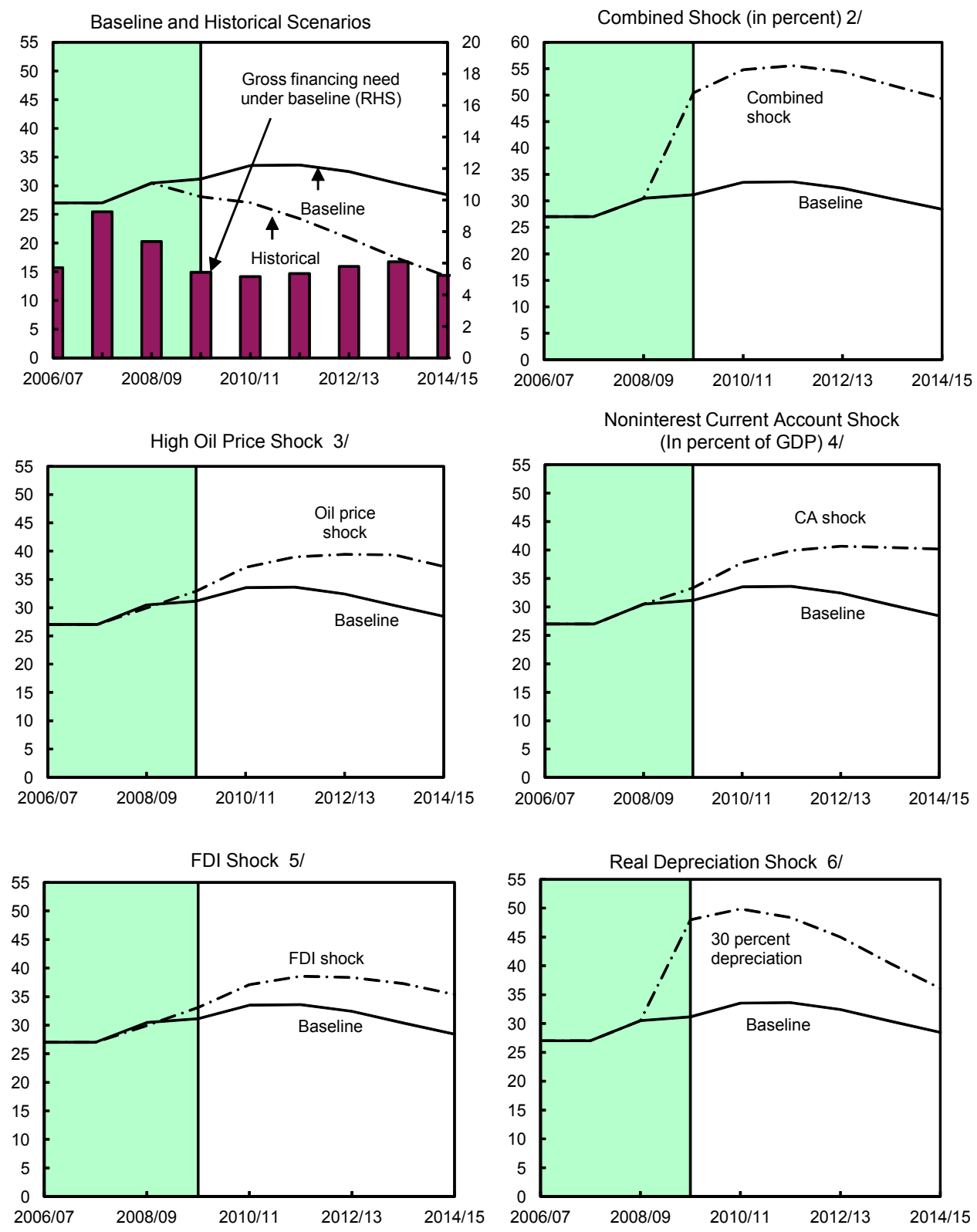

Sources: Historical data from Pakistani authorities; and Fund staff estimates and projections,

1/ Shaded areas represent actual data. Individual shocks are permanent one-half standard deviation shocks. Figures in the boxes represent average projections for the respective variables in the baseline and scenario being presented. Ten-year historical average for the variable is also shown.

2/ Combined impact of shocks to growth, current account, FDI, and real depreciation.

$3 /$ Oil prices are higher by $\$ 10$ for the projection period relative to the baseline.

4/ Current account deficit higher than the baseline scenario by half of the ten-year standard deviation.

$5 /$ Net flows of foreign direct investment are 50 percent lower than in the baseline scenario.

6 / One-time real depreciation of 30 percent occurs in 2009/10. 
Table 1. Pakistan: External Debt Sustainability Framework, 2004/05-2014/15 (In percent of GDP, unless otherwise indicated)

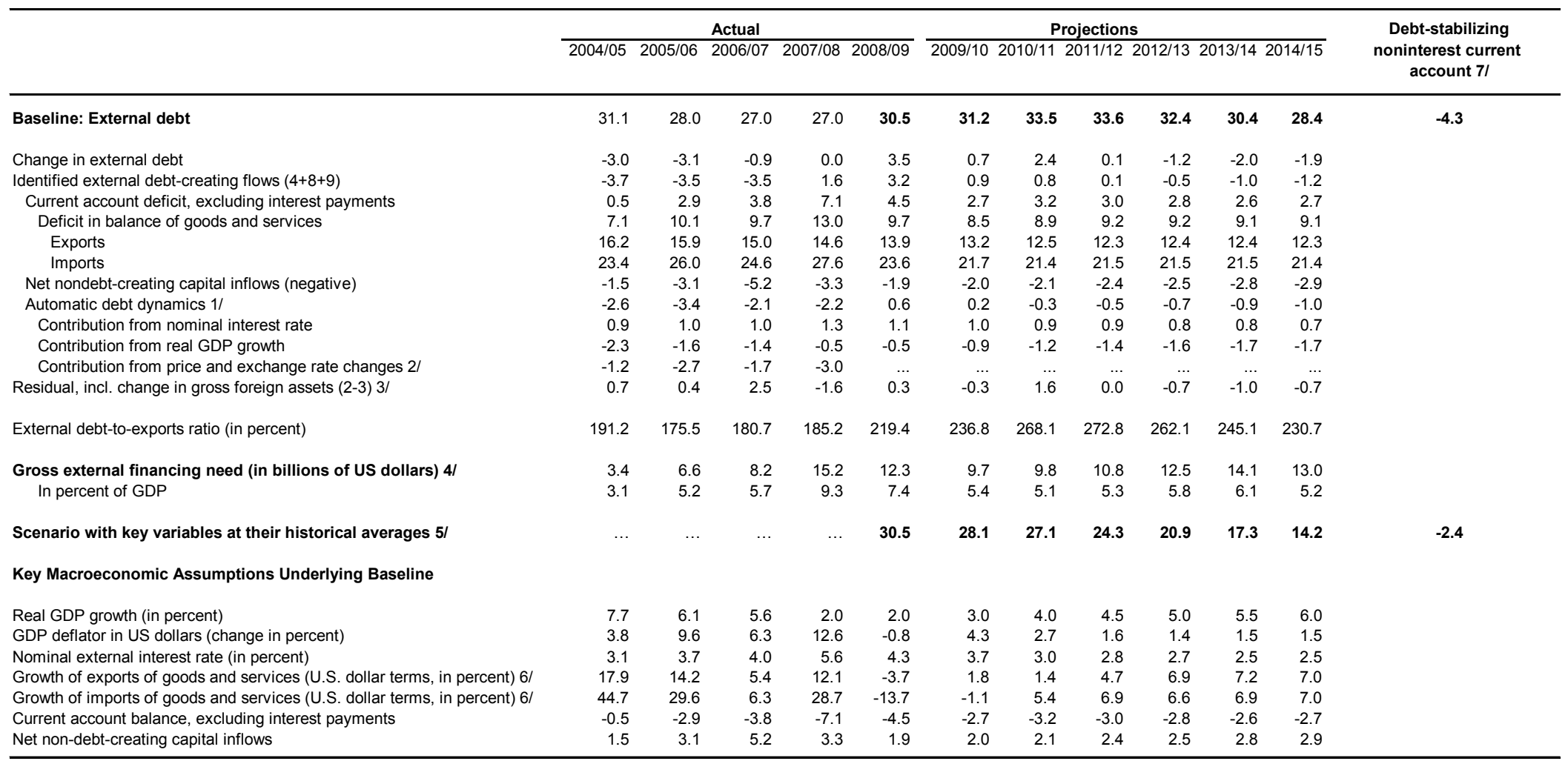

\section{Sources: Pakistani authorities; and Fund staff estimates and projections.}

1/ Derived as $[r-g-\rho(1+g)+e a(1+r)] /(1+g+\rho+g \rho)$ times previous period debt stock, with $r=$ nominal effective interest rate on external debt; $\rho=$ change in domestic GDP deflator in U.S. dollar terms, $\mathrm{g}=$ real GDP growth rate, $\mathrm{e}=$ nominal appreciation (increase in dollar value of domestic currency), and $\mathrm{a}=$ share of domestic-currency denominated debt in total external debt.

$2 /$ The contribution from price and exchange rate changes is defined as $[-\rho(1+g)+e a(1+r)] /(1+g+\rho+g \rho)$ times previous period debt stock. rho increases with an appreciating domestic currency $(\mathrm{e}>0)$ and rising inflation (based on GDP deflator).

$3 /$ For projection, line includes the impact of price and exchange rate changes.

4/ Defined as current account deficit, plus amortization on medium- and long-term debt, plus short-term debt at end of previous period.

5/ The key variables include real GDP growth; nominal interest rate; dollar deflator growth; and both non-interest current account and non-debt inflows in percent of GDP.

6/ Exports and imports of goods and services

7/ Long-run, constant balance that stabilizes the debt ratio assuming that key variables (real GDP growth, nominal interest rate, dollar deflator growth, and non-debt inflows in percent of GDP)

remain at their levels of the last projection year. 
Figure 2. Pakistan: Public Debt Sustainability-Bound Tests $1 /$
(Public debt in percent of GDP)
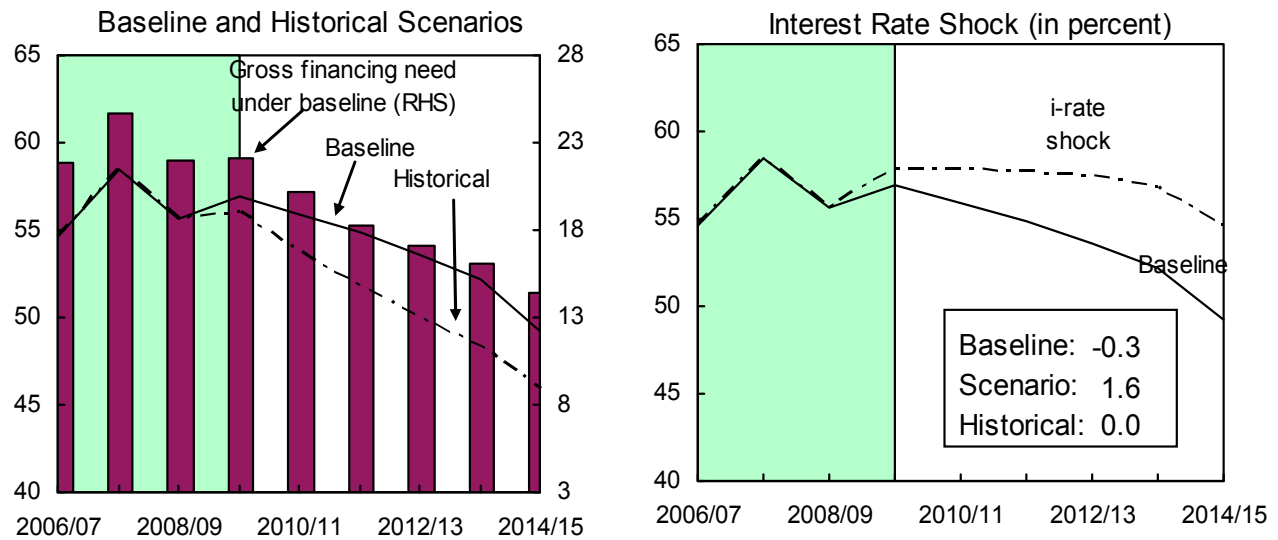

Growth Shock (in percent per year)

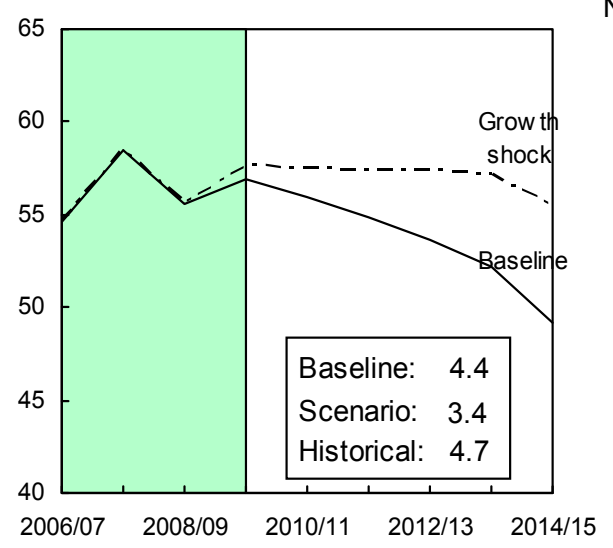

Primary Balance Shock (in percent of GDP) and No policy Change Scenario (constant primary balance)
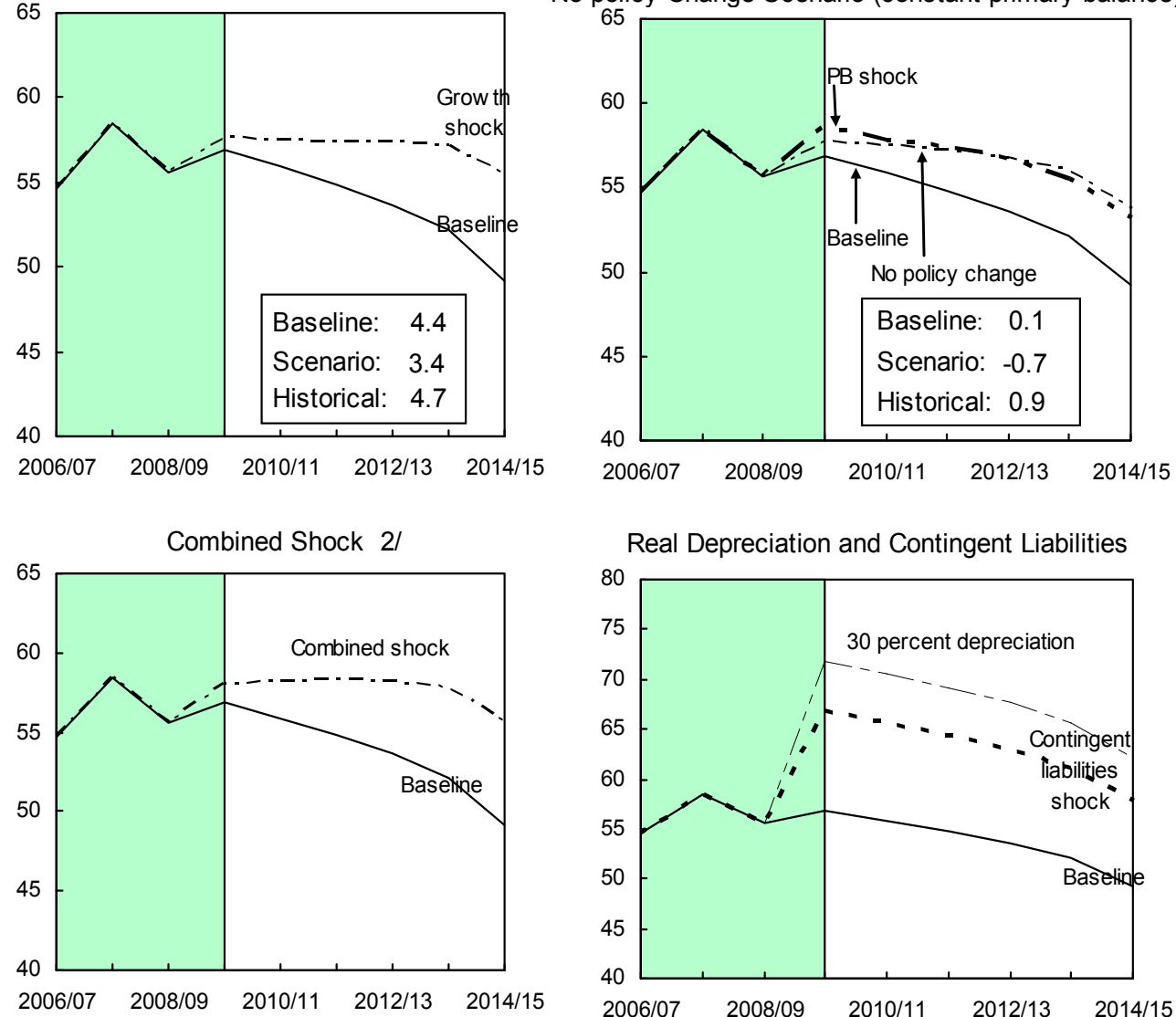

Real Depreciation and Contingent Liabilities

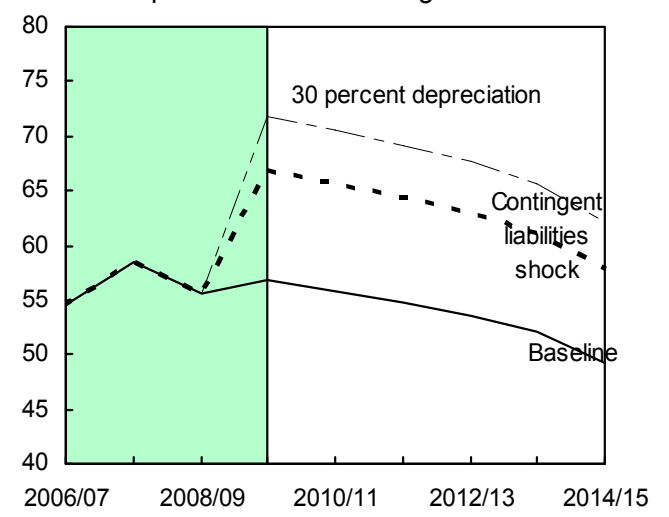

Source: Fund staff estimates and projections.

1/ Shaded areas represent actual data. Individual shocks are permanent one-half standard deviation shocks. Figures in the boxes represent average projections for the respective variables in the baseline and scenario being presented. Ten-year historical average for the variable is also shown. 2/ Permanent $1 / 4$ standard deviation shocks applied to real interest rate, growth rate, and primary balance.

$3 /$ One-time real depreciation of 30 percent and 10 percent of GDP shock to contingent liabilities occur in 2009/10, with real depreciation defined as nominal depreciation (measured by percentage fall in dollar value of local currency) minus domestic inflation (based on GDP deflator). 
Table 2. Pakistan: Public Sector Debt Sustainability Framework, 2004/05-2014/15

(In percent of GDP, unless otherwise indicated)

\begin{tabular}{|c|c|c|c|c|c|c|c|c|c|c|c|c|}
\hline \multirow{3}{*}{$\begin{array}{l}\text { Baseline: Public sector debt } 1 / \\
\text { Of which: Foreign-currency denominated }\end{array}$} & \multicolumn{5}{|c|}{$\begin{array}{c}\text { Actual } \\
\text { 2004/05 2005/06 2006/07 2007/08 2008/09 }\end{array}$} & \multicolumn{6}{|c|}{$\frac{\text { Projections }}{2009 / 10 \quad 2010 / 112011 / 122012 / 132013 / 14 \quad 2014 / 15}$} & \multirow{2}{*}{$\begin{array}{c}\begin{array}{r}\text { Debt-stabilizing } \\
\text { primary balance } 9 /\end{array} \\
-1.4\end{array}$} \\
\hline & 62.0 & 56.4 & 54.6 & 58.4 & 55.6 & 56.4 & 55.3 & 54.1 & 52.7 & 51.2 & 48.1 & \\
\hline & 28.9 & 25.9 & 24.7 & 26.7 & 26.2 & 25.6 & 25.9 & 26.6 & 26.9 & 26.8 & 26.0 & \\
\hline Change in public sector debt & -6.3 & -5.6 & -1.7 & 3.8 & -2.8 & 0.8 & -1.1 & -1.2 & -1.4 & -1.6 & -3.1 & \\
\hline Identified debt-creating flows $(4+7+12)$ & -4.3 & -5.0 & -2.6 & 1.1 & -2.4 & -2.5 & -1.8 & -2.2 & -2.4 & -2.5 & -3.2 & \\
\hline Primary deficit & -0.2 & 0.6 & -0.2 & 2.5 & 0.1 & 0.2 & 0.1 & -0.4 & -0.3 & -0.2 & -0.5 & \\
\hline Revenue and grants & 14.1 & 14.7 & 15.3 & 14.9 & 14.3 & 14.9 & 15.4 & 15.9 & 16.2 & 16.6 & 16.9 & \\
\hline Primary (noninterest) expenditure & 14.0 & 15.3 & 15.1 & 17.4 & 14.5 & 15.1 & 15.5 & 15.5 & 15.9 & 16.4 & 16.4 & \\
\hline Automatic debt dynamics $2 /$ & -5.0 & -5.8 & -2.4 & -1.1 & -3.5 & -2.8 & -2.2 & -2.1 & -2.4 & -2.7 & -3.1 & \\
\hline Contribution from interest rate/growth differential $3 /$ & -5.8 & -6.0 & -2.6 & -3.8 & -7.7 & -2.8 & -2.2 & -2.1 & -2.4 & -2.7 & -3.1 & \\
\hline Of which: Contribution from real interest rate & -1.2 & -2.8 & 0.2 & -2.9 & -6.8 & -1.3 & -0.2 & 0.1 & 0.1 & -0.1 & -0.4 & \\
\hline Of which: Contribution from real GDP growth & -4.5 & -3.2 & -2.8 & -0.9 & -0.9 & -1.5 & -2.0 & -2.2 & -2.4 & -2.6 & -2.7 & \\
\hline Contribution from exchange rate depreciation $4 /$ & 0.8 & 0.2 & 0.1 & 2.7 & 4.2 & & & & & & & \\
\hline Other identified debt-creating flows & 0.9 & 0.2 & 0.1 & -0.3 & 1.0 & 0.2 & 0.3 & 0.3 & 0.3 & 0.4 & 0.4 & \\
\hline Privatization receipts (negative) & -0.4 & -1.3 & -0.8 & 0.0 & 0.0 & -0.4 & -0.2 & -0.1 & -0.1 & -0.1 & -0.1 & \\
\hline Recognition of implicit or contingent liabilities & 0.0 & 0.0 & 0.0 & 0.0 & 0.0 & 0.0 & 0.0 & 0.0 & 0.0 & 0.0 & 0.0 & \\
\hline Build-up of bank deposits & 1.3 & 1.5 & 0.9 & -0.3 & 1.0 & 0.6 & 0.5 & 0.5 & 0.5 & 0.5 & 0.5 & \\
\hline Residual, including other asset changes (2-3) 5 / & -2.0 & -0.6 & 0.9 & 2.7 & -0.5 & 3.3 & 0.7 & 1.0 & 1.0 & 0.9 & 0.1 & \\
\hline Public sector debt-to-revenue ratio $1 /$ & 438.2 & 383.3 & 357.1 & 392.8 & 388.0 & 377.8 & 358.1 & 340.9 & 324.9 & 308.2 & 284.0 & \\
\hline Gross financing need 6/ & 19.0 & 20.7 & 21.9 & 24.7 & 22.0 & 21.8 & 19.8 & 18.0 & 16.9 & 15.8 & 14.1 & \\
\hline In billions of U.S. dollars & 20.8 & 26.4 & 31.4 & 40.7 & 36.6 & 39.0 & 37.9 & 36.6 & 36.4 & 36.6 & 35.1 & \\
\hline Scenario with key variables at their historical aver & $\ldots$ & $\ldots$ & $\ldots$ & $\ldots$ & 55.6 & 55.6 & 53.2 & 51.2 & 49.2 & 47.3 & 44.8 & -0.9 \\
\hline Scenario with no policy change (constant primary balance) in 2009/10-2013/14 & $\ldots$ & $\ldots$ & $\ldots$ & $\ldots$ & 55.6 & 58.2 & 57.3 & 56.9 & 56.2 & 55.0 & 52.6 & -1.3 \\
\hline \multicolumn{13}{|l|}{ Key Macroeconomic and Fiscal Assumptions Underlying Baseline } \\
\hline Real GDP growth at market prices (in percent) & 7.7 & 6.1 & 5.6 & 2.0 & 2.0 & 3.0 & 4.0 & 4.5 & 5.0 & 5.5 & 6.0 & \\
\hline Average nominal interest rate on public debt (in percent) 8 & 5.5 & 5.9 & 8.6 & 10.3 & 10.6 & 9.1 & 7.5 & 7.0 & 6.5 & 6.0 & 5.5 & \\
\hline Average real interest rate (nominal rate minus change in GDP deflator, in percent) & -1.6 & -4.6 & 0.9 & -5.9 & -14.3 & -2.4 & 0.0 & 0.5 & 0.5 & 0.0 & -0.5 & \\
\hline Nominal appreciation (increase in U.S. dollar value of local currency, in percent) & -2.5 & -0.9 & -0.5 & -11.4 & -74.0 & -2.4 & 0.0 & 0.0 & .0 & 0.0 & 0.0 & \\
\hline Inflation rate (GDP deflator, in percent) & 7.0 & 10.5 & 7.7 & 16.2 & 24.9 & 11.5 & 7.5 & 6.5 & 6.0 & 6.0 & 6.0 & \\
\hline Growth of real primary spending (deflated by GDP deflator, in percent) & 16.5 & 16.2 & 4.2 & 17.8 & -15.2 & 7.7 & 6.7 & 4.3 & 7.7 & 9.0 & 6.0 & \\
\hline Primary deficit & -0.2 & 0.6 & -0.2 & 2.5 & 0.1 & 0.2 & 0.1 & -0.4 & -0.3 & -0.2 & -0.5 & \\
\hline
\end{tabular}

Sources: Pakistani authorities; and Fund staff estimates and projections.

1/ General government gross debt: excludes obligations to the IMF except budget financing, external military debt, commercial debt, short-term loans, and debt of public enterprises.

2/ Derived as $[(r-p(1+g)-g+a e(1+r)](1+g+p+g p))$ times previous period debt ratio, with $r=$ interest rate; $p=$ growth rate of GDP deflator; $g=$ real GDP growth rate; $a=$ share of foreign-currency

denominated debt; and $\mathrm{e}=$ nominal exchange rate depreciation (measured by increase in local currency value of U.S. dollar).

$3 /$ The real interest rate contribution is derived from the denominator in footnote $2 /$ as $r-\pi(1+g)$ and the real growth contribution as $-g$.

4/ The exchange rate contribution is derived from the numerator in footnote $2 /$ as ae $(1+r)$.

5/ For projections, his line includes exchange rate changes. For 200/10, it relects, inter alia, the assumption by the government of electricity sector debt of Rs. 277 billion (1.9 percent of GDP).

/ Defined as public sector defict, plus amortization of medium and long-lerm public sector debt, plus shor-term debt at end of previous period.

The

9/ Assumes that key variables (real GDP growth, real interest rate, and other identified debt-creating flows) remain at the level of the last projection year. 


\section{ATTACHMENT I. LETTER OF INTENT}

May 3, 2010

Mr. Dominique Strauss-Kahn

Managing Director

International Monetary Fund

Washington, D.C. 20431

Dear Mr. Strauss-Kahn:

We recently held discussions with Fund staff on the fourth review under the Stand-By Arrangement (SBA). Based on these discussions, we have updated our economic program for 2009/10, as reflected in the attached Fourth Supplementary Memorandum of Economic and Financial Policies (SMEFP). In support of our policies, the Government of Pakistan requests that the Executive Board of the Fund complete the fourth review and approve the fifth purchase under the SBA in an amount equivalent to SDR 766.7 million.

We have made further progress under our stabilization program, notwithstanding challenging circumstances. An economic recovery is underway, and our external position has improved further. We have corrected nearly all of the fiscal slippage from the first quarter of 2009/10 in the second quarter when all quantitative performance criteria for end-December 2009 were observed, except for the budget deficit target, which was exceeded by 0.02 percent of GDP, and made progress in preparing for the introduction of the value added tax on July 1, 2010. However, our fiscal policy continues to be challenged by high security outlays and shortfalls in external financing, and we consider it necessary to adjust our fiscal program for the remainder of 2009/10. We believe that the policies set out in the attached SMEFP are adequate to achieve the objective of the program through further consolidation of macroeconomic stability and advance our ambitious reform agenda. However, we stand ready to take any additional measures deemed appropriate for this purpose, and will consult with the Fund on the adoption of these measures in accordance with Fund policies on such consultations. We will also provide the Fund with the information it may request to monitor progress in implementing economic and financial policies.

We request waivers of nonobservance for the end-March quantitative performance criteria on the overall budget deficit (excluding grants) and net government borrowing from the State Bank of Pakistan (SBP). Although final data for this quarter are not yet available, preliminary information indicates that we missed the adjusted end-March quantitative performance criterion on the fiscal deficit by 0.4 percent of GDP, on account of lower revenue and security-related expenditures and shortfalls in disbursements of IDP grants and Tokyo-related multilateral assistance. We also missed the end-March quantitative performance criterion on net government borrowing from the SBP by 0.2 percent of GDP due to a delay in the disbursement of external financing to the budget. We plan to strengthen government liquidity management and reduce recourse to the central bank overdraft to ensure we meet end-quarter targets for net borrowing from the SBP. We also request rephasing of access under the arrangement. To this effect, we propose that the last three purchases be consolidated into two and that these (sixth and seventh) purchases increased to SDR 1,150.05 million and SDR 1,149.815 million, respectively. 
We request that August 15, 2010 be the availability date for the sixth purchase, conditional on the completion of the fifth program review and observance of end-June 2010 performance criteria, and November 15, 2010 be the availability date for the seventh purchase, conditional on the completion of the sixth and final review and observance of end-September 2010 performance criteria, which would be set at the time of the fifth review.

Furthermore, we request: (i) a modification of the end-June 2010 performance criteria for the budget deficit to increase the cumulative end-quarter ceilings by Rs 22 billion $(0.15$ percent of GDP) in order to allow space for urgent security outlays and avoid undue cuts in other priority spending; and (ii) a modification of the end-June 2010 performance criterion (raising the floor) for the net foreign assets of the SBP by $\$ 300$ million, taking into account our strengthened external position.

We consent to the publication of this letter, the attached SMEFP, the revised Technical Memorandum of Understanding, and the related staff report.

Sincerely yours,

$$
/ \mathrm{s} /
$$

Abdul Hafeez Shaikh

Advisor to the Prime Minister on Finance, Revenue, Economic Affairs and Statistics

\author{
/s/ \\ Syed Salim Raza \\ Governor \\ State Bank of Pakistan
}

\section{Attachments:}

Fourth Supplementary Memorandum of Economic and Financial Policies Technical Memorandum of Understanding 


\section{Attachment II. Pakistan: Fourth Supplementary Memorandum on ECONOMIC AND FinANCIAL POLICIES FOR 2009/10}

May 3, 2010

\section{INTRODUCTION}

1. This memorandum supplements our Memorandum on Economic and Financial Policies (MEFP) for 2008/09-09/10 of November 20, 2008 and the first, second, and third supplements thereto dated March 16, July 29, and December 11, 2009, respectively. It describes policy implementation to date and lays out additional policies agreed in the context of the fourth review under the Stand-By Arrangement (SBA).

\section{RECENT ECONOMIC DEVELOPMENTS}

2. Despite significant internal security challenges, our stabilization program is on track, but risks to the fiscal outlook are rising. Headline inflation declined from 25 percent in October 2008 to 8.9 percent in October 2009, but increased to 12.9 percent in March 2010. The rupee has remained stable at around Rs 84-85 per U.S. dollar in recent months, gross foreign exchange reserves reached $\$ 11$ billion in late March, 2010, and market demand for treasury bills has remained healthy. All quantitative performance criteria for end-December 2009 were observed (Table 1), except for the budget deficit target, which was exceeded by 0.02 percent of GDP. Structural reforms are progressing despite extremely challenging circumstances relating mainly to a deterioration of the internal security situation and an intensification of the military campaigns in the northwest. We have submitted VAT legislation to the parliament and provincial assemblies. Further, we have submitted to parliament the amendments to the State Bank of Pakistan (SBP) Act; tax administration reforms are continuing; the amendments to the Banking Companies Ordinance have been approved by the National Assembly; and Anti-Money Laundering legislation was approved on March 26. Electricity reform has proceeded, albeit slower than planned. We continue to face challenges to our stabilization program due to a widening of the military operations, revenue shortfalls and delays in disbursements of pledged donor support, which has complicated fiscal management, while inflation has picked up.

\section{There are some signs that growth is starting to recover. Large-scale}

manufacturing output has started to increase after a protracted decline, and the improvement in the global economy has improved the outlook for manufacturing exports. Agriculture performance is mixed, with lower rice and sugarcane output, offset by a stable outlook for wheat and higher cotton output. Private sector credit growth has picked up somewhat as businesses rebuild their working capital. Financial markets remain positive. The stock market index has risen in recent months and the spreads on Pakistan sovereign bonds have stabilized. However, the growth outlook is subject to risks: most prominently the domestic security situation and reliability of electricity supply, as well as the pace of global economic recovery. 
4. Fiscal performance was affected by weak economic activity, security challenges, and shortfalls of external support. The overall fiscal performance in the first half of 2009/10 was satisfactory albeit with lower revenue collection leading to constrained expenditures. The tax revenue collection targets were missed by about 0.2 percent of GDP in the first half of 2009/10. Also, nontax revenue was about 0.2 percent of GDP lower than projected. Expenditure controls helped limit the budget deficit. However, revenue shortfalls increased in the third quarter, while the security-related spending pressures limited the scope for expenditure cuts. As a result, the end-March budget deficit target was missed by about 0.2 percent of GDP. Shortfalls of external support increased that margin (via adjustors) to an estimated 0.4 percent of GDP.

\section{Monetary policy has tried to balance price stability with the need to support a}

nascent recovery. The discount rate was cut by 50 bps to 12.5 percent in November, but in January, the SBP kept it unchanged in view of sticky core inflation, the uncertain fiscal outlook, with greater domestic financing needs to compensate for external financing shortfalls, and expected inflationary pressures from higher energy and food price. In March, the SBP also kept the discount rate unchanged, trying to strike a difficult balance between the imperatives of reducing inflation, ensuring financial stability, and supporting economic recovery.

6. $\quad$ Fiscal challenges have prompted us to develop compensatory measures. The measures, which include both expenditure and revenue measures, will help safeguard the macroeconomic stability and reduce inflation. These measures provide the needed fiscal space for higher security spending and to protect social spending, including for internally displaced persons (IDPs), while safeguarding macroeconomic stability. It remains critical that donor support pledged during the donors conference in Tokyo in April 2009 is disbursed in a timely manner.

\section{POLICIES FOR THE ReMAINDER OF 2009/10}

\section{The macro-economic backdrop for our program remains largely unchanged.}

Real GDP is projected to increase by 3 percent, with somewhat stronger manufacturing output balancing a mixed outlook for the agriculture sector. Electricity tariff adjustments and higher food prices will push annual consumer price inflation up, but inflation is expected to decline and we project year-on-year headline inflation in June will be 12 percent. The current account deficit is expected to improve further to $3 \frac{3}{4}$ percent of GDP supported by lower imports, despite higher oil prices, lower interest and profit outflows, and strong remittance inflows, but the robustness of the balance of payments position will be heavily influenced by donor disbursements.

8. $\quad$ Fiscal risks are a threat to macroeconomic stability. The risks stem mainly from possible shortfalls in revenue collection and donor support pledged in Tokyo in April 2009, as well as expenditure pressure largely security-related. We have identified contingency measures of 0.3 percent of GDP that could address these risks. The measures comprise non-priority development expenditure cuts. As noted, we are determined to safeguard pro-poor spending and spending for IDPs. 


\section{A. Fiscal Policy}

9. The fiscal deficit target for 2009/10 will be adjusted to 5.1 percent of GDP. The deficit will be financed by higher than projected privatization inflows, which will more than compensate for the shortfalls in IDP grants and foreign financing.

10. There are risks that revenue (both tax and non-tax) may be lower than expected. The government plans to implement the following measures to head-off these risks, and stands ready to take additional measures as required:

- $\quad$ Revenue measures: The 2009/10 target for Federal Board of Revenue (FBR) collections has been revised down somewhat to Rs 1,380 billion, which takes into account, in part, the shortfall in the first three quarters of the year. Tax collection is being monitored closely and an increase in collections is expected in the fourth quarter reflecting both greater collection efforts and collection of two advance installments in the fourth quarter. Further, additional inflows from the U.S. Coalition Support Fund are expected in the fourth quarter.

- $\quad$ Expenditure measures: Given the pressing need for higher security spending, we have found offsetting savings. To offset the impacts of revenue and external financing shortfalls we will cut non-priority current and development spending. We believe we have adequate provisions for security and IDP outlays and, if needed, we will meet any additional fiscal shocks through additional restraint of non-priority spending.

11. We have taken necessary steps towards the introduction of a broad-based VAT by July 1, 2010. A Federal VAT act was submitted to the Ministry of Law and Parliamentary Affairs on $31^{\text {st }}$ December 2009, after approval of the Prime Minister. The draft Federal VAT Act was submitted to the National Assembly on February 25 for discussion, was submitted to the Senate on March 1, and was discussed by the Standing Committee of the Senate on March 17-18, which has completed its consideration of the bill and passed it on to the National Assembly. Provincial VAT Acts, consistent with each other and the Federal Act, have been prepared, discussed with provincial governments, and submitted to provincial assemblies in late-March, after preliminary parliamentary discussions on the federal component. The integrity and consistency of the VAT Acts will be ensured and they will be implemented as an integrated national VAT to avoid the problem of cascading and tax competition. To this end, the Prime Minister has set up a Committee chaired by the Minister of State for Finance and Revenue comprising the Federal Finance Secretary and all provincial Chief Secretaries to resolve issues related to the operation of provincial VAT. The review of the VAT law, including zero-rating and exemptions, is expected to be completed by the finance and revenue committees of the parliament by mid-May 2010. Initial estimates have been prepared on the assessment of the revenue impact of VAT and we will refine them further in the coming weeks. The VAT Acts will be supported by an adequate risk-based audit mechanism and be free of exemptions and domestic zero-ratings except those that are indicated by international best practice. The Federal and Provincial VAT Acts are expected to pass by May 31, 2010. As a preparatory step toward full implementation of the VAT by July 1, 2010, VAT 
regulations are being prepared and will be issued two to three weeks after enactment of the Federal VAT Act. A review of business processes, recordkeeping, and design of forms will be an integral component of the VAT regulations. We will also submit legislation to harmonize the existing tax laws with the VAT act in early June, 2010. We are receiving assistance from the IMF and World Bank to introduce the VAT.

12. The following actions to improve tax administration and its governance will be taken:

- We are redoubling our efforts to reduce the number of non-filers and underreporting taxpayers. Around 200,000 letters were sent to non-filers and underreporters in addition to text messages and a media campaign, which resulted in 121,000 taxpayers filing before the extended deadline of January 25, 2010. Taxpayers who have not filed are receiving statutory notices and the department will enforce the filing of returns by non-filers, who receive income from property rents and have commercial electricity connections. If they still do not comply, the FBR will conduct a provisional assessment based on the information available and tax accordingly.

- Major efforts are being made to collect tax arrears. We are focusing on resolution of stuck up revenues especially in the oil and insurance sectors. In addition, we are making efforts to recover withholding taxes in the banking and insurance sectors, which have not been deposited through reconciliation of accounts.

- Tax audits are moving ahead. We have identified over 900 companies and associations of persons for audit. 468 of these audits have been outsourced to the Institute of Chartered Accountants Pakistan, which has been provided an auditing framework by FBR. We expect the majority of these audits will be completed by June 2010. The remaining audits will be conducted by FBR and (most of these) will be completed by May 2010 .

- We are making progress in strengthening the system of tax refunds. Software has been developed and has been successfully pilot tested in the Large Taxpayer Unit of Islamabad. It has been agreed with banks that the refund system would be launched in conjunction with the introduction of a VAT in 2010/11. To this effect, on March 29, the FBR issued a notification allowing the expeditious processing and payment of refunds to manufacturing exporters registered at the Lahore Regional Taxpayers Office (RTO) from tax period April, 2010 and to all manufacturing exporters registered at other RTOs and LTUs from tax period July, 2010. As part of the preparatory effort for the refund system, we have already put in place system development and related procedures. This was done based on the recommendations for changes in the sales tax refund rules and risk criteria made by the committee that was set up for this purpose. After the introduction of suitable changes to mitigate any remaining risk factors, the system will be ready for deployment by end-June, 2010. 
13. We are continuing the roll out of the Benazir Income Support Program (BISP).

We have completed the pilot for introducing the new targeting mechanism, using a poverty-scorecard, carried out in 15 districts and covering 2.1 million people. Based on the results of the pilot, the cut-off score (used to determine eligibility) for the povertyscorecard has been fixed in January 2010. Further, we have started the process to exclude those beneficiaries, who are above the cut-off score, from receiving benefits under the new targeting mechanism in the 15 districts covered by the pilot. Poverty-scorecard data collection in Baluchistan is being started on a priority basis. A comprehensive plan for nationwide rollout of the poverty-scorecard based targeting has been prepared with the help of the World Bank; the process of contracting firms has begun and is expected to be completed by end-May 2010. The poverty-scorecard system expected to be rolled out nationwide by end-March 2011. The delays in the rollout of the poverty-scorecard system will slow delivery of BISP assistance and we now project BISP disbursements of Rs 50 billion in 2009/10.

\section{We are making progress towards eliminating electricity tariff differential}

subsidies. Electricity tariffs were adjusted in December to make up for the shortfall in the October 1 increase, which was below the 6 percent agreed with the World Bank and the Asian Development Bank. Subsequently, an additional 12 percent tariff increase was implemented on January 1, 2010, as scheduled. Tariffs will be increased by 6 percent to take effect from April 1 in accordance with earlier plans. We have also corrected the measures applied by NEPRA to fully recover the power purchase costs through the monthly adjustments; and put in place a program of recovering the unrecovered power purchase costs from the first nine months of this fiscal year over a 15-month period beginning April 2010. We expect to complete discussions with the World Bank and the Asian Development Bank staffs and agree before end-June 2010 on a framework for the power sector that would ensure no tariff differential subsidies. This will require restoring gas supplied to the power sector by a minimum of an additional 183 million cubic feet per day (mmcfd) for at least nine months. In addition, we have also initiated measures, which we plan to put in place prior to the next review, to improve governance and financial discipline in the power sector and once and for all eliminate the circular debt induced by the power sector's financial performance. Following the April 20-22 Energy Summit, the government has announced measures to increase use of conservation, augment gas supplies to the power sector to improve the supply capacity and make it more cost efficient, and improve governance.

\section{We are taking steps to clear the remaining stock of circular debt in the}

electricity sector. The transfer of the bulk of the remaining stock of debt of electricity companies (Rs 216 billion) is expected to take place in May, 2010. The audit of this pre-2009/10 debt has been finalized. The government will continue to pay interest on this debt. More recently, the office of the Federal Adjustor has been re-established to clear payment arrears of government agencies and departments more efficiently by making at-source deductions from the budget. 
16. We will address the quasi-fiscal problems with commodity operations. The stock of outstanding wheat commodity credits was reduced by Rs 42 billion between January 31 and March 31, 2010, ahead of the 2010 procurement season, to avoid crowding out the private sector. Losses for government procurement agencies will be covered from the budget and not left to accumulate on the agencies' books. For the 2010 procurement season, we will remain within our announced procurement target of 6.5 million tones.

\section{Meeting our fiscal program's objectives hinges importantly on prudent fiscal} behavior at the provincial level. To that effect, we have taken steps to ensure that borrowing by provinces is limited and consistent with the fiscal targets of the general government. Monthly meetings between the federal and provincial Finance Secretaries have been instituted. We are continuing to monitor closely provincial borrowing and ensuring they stay within their established Ways and Means limits of six weeks of wages and salaries.

18. Pakistan's fiscal transparency is being enhanced. In 2009, the Ministry of Finance began to publish on its web site the amount of newly issued government guarantees, and in March 2010, we started publishing also information about the total stock of outstanding government guarantees.

19. A medium-term budget framework is now in place. This framework aims at ensuring fiscal prudence and consolidation and to lay the basis for sustainable growth. It will support better expenditure planning and predictability of resource availability. We have prepared and obtained approval of the Cabinet of a medium-term budget strategy paper (BSP-1) for 2010/11-2012/13 that sets the fiscal framework and budget priorities for this period, and sets indicative budget ceilings for the major expenditure categories. It also takes account of the new revenue-sharing arrangements between the federal and provincial governments agreed by the National Finance Commission (NFC) in December 2009. The NFC agreed that provinces would receive a larger share of tax revenue in 2010/11-2014/15 and a concomitant increased responsibility for government functions.

The Constitutional Reforms Committee has analyzed functions that will be transferred to provinces and has presented its report and recommendations, which have been enacted as constitutional amendments in April 2010.

\section{We remain committed to the transition to a Treasury Single Account by}

end-June 2010. We initiated the survey of deposits. We are collecting information on commercial bank deposits of federal entities, and will have all non-own source, nonsecurity related cash balances transferred to the Federal Consolidated Fund by end-June 2010 and associated accounts closed. We will ensure these transfers do not affect the liquidity of the banking system. We will ensure that a minimum interest rate is received on all federal government deposits.

21. The government has initiated the restructuring of large public sector enterprises (PSEs). As a first step, a Cabinet Committee on Restructuring (CCOR) has been constituted under the Advisor to the Prime Minister on Finance, Revenue, Economic Affairs and Statistics. In its first meeting, held on February 8, 2010 the CCOR approved 
an overall framework for restructuring of eight major PSEs. ${ }^{1}$ The framework entails formation of independent and professionally qualified board of directors for each PSE, and the hiring of professional CEOs from the private sector. The new management of each PSE will be tasked to produce a viable restructuring plan, which will be vetted and approved by CCOR. The appointment of new boards and hiring CEOs was initiated in March.

\section{B. Monetary and Exchange Rate Policies}

\section{Monetary policy will continue to focus primarily on price stability, while} building international reserves. Lower inflation will support the nascent revival in economic activity and pave the way for lower interest rates. Inflation has been more persistent than expected, due to higher administered prices and higher inflationary expectations taking hold. The SBP will continue to monitor inflation carefully, and if inflationary pressures persist, it will tighten monetary policy as needed.

\section{The new liquidity management framework has reduced the volatility in the} overnight money market repo rate. The interest rate corridor framework has improved the anchoring of short-term rates with reduced volatility in the weighted average overnight repo rate, which averaged around 12 percent year since July 2009. To enhance the functioning of the interest rate corridor framework, the SBP may consider measures related to reserve requirements and associated maintenance period as appropriate. The SBP and the Finance Division will continue to enhance coordination and communication on budgetary flows to strengthen further liquidity forecasting.

\section{We will continue to pursue a flexible exchange rate policy to safeguard the} external position and competitiveness. Interventions in foreign exchange market will continue to be aimed at achieving program NFA targets. The SBP phased out provisions of foreign exchange for crude oil imports in December 2009, and the transition has been effected smoothly with the market functioning appropriately. The balance of payments position has strengthened sufficiently and expected to improve further.

\section{Financial Sector and Safeguards Issues}

\section{We continue to work toward strengthening the soundness of the financial} sector. Preliminary data indicate that Financial Soundness Indicators through endDecember deteriorated a little, but the system remains well capitalized. Although nonperforming loans (NPLs) continued to increase in the quarter ended December 2009, their rate of increase slowed compared to previous quarters. Further, credit increased in this quarter and as a result NPL-to-loans ratio at end-December improved to 12.2 percent. However, capital ratios weakened slightly: the risk-weighted capital-to-assets ratio declined from 14.3 to 14.1 percent and the (un-weighted) capital-to-assets ratio dropped

\footnotetext{
${ }^{1}$ They are Pakistan International Airlines, Pakistan Electric Power Company, Pakistan Railways, Pakistan Steel Mills, National Highway Authority, Pakistan Agricultural Storage and Services Corporation, Trading Corporation of Pakistan, and Utility Stores Corporation.
} 
from 10.5 to 10.1 percent. The restructuring of NPLs needs to be expedited to bring their level down. Eight banks did not comply with the higher end-2009 minimum capital requirement, however, these are expected to be brought into compliance soon either through capital injections or mergers. Exposure to Dubai appears to be very limited and any losses from the Dubai World restructuring can be covered from capital.

\section{The National Assembly has passed amendments to the Banking Companies} Ordinance that strengthens the SBP's enforcement powers to deal with problem banks. The National Assembly has approved the amendments and Senate approval is expected in early May. The amendments empower the SBP to appoint administrators and allow it to restructure problem banks in a variety of ways either through administrators or by itself. They also empower the SBP to cancel or write down capital of the insolvent banks, prohibit banks from paying dividends or accepting fresh deposits, and compel any undesirable major shareholders to transfer their shares to fit and proper persons. The amendments would enhance the legal basis for the SBP to deal with problem banks.

\section{Amendments to the SBP law to enhance the SBP's operational independence} have been submitted to Parliament on March 17. They are scheduled to be considered by the National Assembly's Committee on Finance and Revenue in the next weeks. Among other things, these amendments will curtail recourse by the budget to SBP credit.

\section{Consistent with the Basel Committee's (Basel 3) reform program, the SBP} would consider the proposed modifications for the banking sector by strengthening the regulatory capital framework. The SBP will conduct, in the near future, an impact assessment of the proposals recently advanced by the Basel Committee. A preliminary analysis suggests that the proposed changes will not have a significant impact on the financial stability indicators in Pakistan. This reflects strong capital adequacy ratios, banks' capital being largely composed of Tier 1 capital, a large share of liquid assets (government securities) in banks' portfolio, and banks limited off-balance sheet exposures.

29. An update to the March 2009 safeguards assessment was completed in February 2010 in relation to the augmentation of access under the current $S B A$. The updated assessment addressed several safeguards measures including the enactment of the amendments to the SBP Act and the confirmation of foreign reserves. As noted, the government has presented amendments of the SBP Act to parliament. Regarding confirmation of foreign reserves, the assessment recommended stronger oversight by the Audit Committee over the external audit procedures related to the independent confirmation of reserve balances for the 2010 audit. The SBP agreed that external auditors may directly confirm the foreign exchange balances from counterparties.

\section{Program Monitoring ANd Financing}

30. The program will remain subject to quarterly program reviews and quarterly performance criteria for 2009/10, as specified in Table 1. Quarterly performance 
criteria for end-June have been revised. Attached is an addendum to the Technical Memorandum of Understanding (TMU) of December 11, 2009.

31. The following measures will be prior actions for Board consideration of the fourth review: Submission of Federal VAT Acts to parliament and consistent Provincial VAT Acts to provincial assemblies.

32. The introduction of the VAT (structural benchmark for July 1, 2010) and reaching understandings on the 2010/11 budget will the key issues for the fifth review.

33. The program remains fully financed but disbursement of external assistance is crucial. 
Table 1. Pakistan: Quantitative Performance Criteria, June 2009-June 2010

\begin{tabular}{|c|c|c|c|c|c|c|}
\hline & $\begin{array}{l}\text { Outst. Stock } \\
\text { end-Sept. } \\
2008\end{array}$ & $\begin{array}{l}\text { end-Jun. } \\
2009\end{array}$ & $\begin{array}{l}\text { end-Sept. } \\
2009\end{array}$ & $\begin{array}{l}\text { end-Dec. } \\
2009\end{array}$ & $\begin{array}{l}2 / 3 / \\
\text { end-Mar. } \\
2010\end{array}$ & $\begin{array}{l}\text { Prog. } 21 \\
\text { end-Jun. } \\
2010\end{array}$ \\
\hline Floor on net foreign assets of the SBP* (stock, in millions of U.S. dollars) & 3,953 & 2,782 & 3,200 & 5,100 & 5,700 & 5,200 \\
\hline Adjusted target $4 /$ & $\ldots$ & 2,428 & 4,406 & 4,816 & 3,830 & \\
\hline Actual & $\ldots$ & 3,982 & 5,706 & 5,360 & 4,752 & \\
\hline Ceiling on net domestic assets of the SBP* (stock, in billions of Pakistani rupees) & 1,250 & 1,314 & 1,300 & 1,270 & 1,220 & 1,320 \\
\hline Adjusted target & $\ldots$ & 1,316 & 1,231 & 1,321 & 1,412 & \\
\hline Actual & $\ldots$ & 1,183 & 1,141 & 1,209 & 1,231 & \\
\hline Ceiling on net government borrowing from $\mathrm{SBP}^{*}$ (stock, in billions of Pakistani rupees) & 1,227 & 1,181 & 1,130 & 1,130 & 1,130 & 1,130 \\
\hline Adjusted target & $\ldots$ & 1,181 & 1,130 & 1,130 & 1,130 & \\
\hline Actual & $\cdots$ & 1,130 & 1,047 & 1,066 & 1,160 & \\
\hline Ceiling on overall budget deficit (cumulative flow, in billions of Pakistani rupees) & 142 & 562 & 194 & 400 & 604 & 762 \\
\hline Adjusted target $4 /$ & $\ldots$ & 562 & 172 & 399 & 576 & \\
\hline Actual & $\cdots$ & 680 & 225 & 402 & 636 & \\
\hline $\begin{array}{l}\text { Ceiling on outstanding stock of short-term public and publicly guaranteed external debt* (in millions } \\
\text { of U.S. dollars) }\end{array}$ & 515 & 4500 & 1500 & 1500 & 1500 & 1500 \\
\hline Actual & $\begin{array}{c}515 \\
\ldots\end{array}$ & 652 & $\begin{array}{c}1,500 \\
589\end{array}$ & $\begin{array}{c}1,500 \\
372\end{array}$ & $\begin{array}{c}1,500 \\
631\end{array}$ & 1,500 \\
\hline 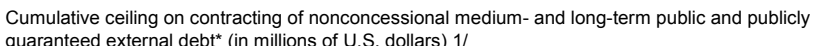 & 9500 & 9.500 & 9.500 & 9500 & 9.500 & 9500 \\
\hline Actual & $\ldots$ & 1,649 & 1,724 & 2,128 & 2,418 & \\
\hline $\begin{array}{l}\text { Accumulation of external payments arrears (continuous performance criterion during the program } \\
\text { period) })^{\star} \text { (in millions of U.S. dollars) }\end{array}$ & . & . & . & 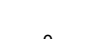 & 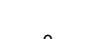 & 0 \\
\hline Actual & ... & 0 & 0 & 0 & 0 & 0 \\
\hline Continuous ceiling on SBP's foreign currency swaps and forward sales* (in millions of U.S. dollars) & 1,900 & 2,750 & 2,500 & 2,500 & 2,500 & 2,500 \\
\hline Actual & $\ldots$ & 1,690 & 2,035 & 2,355 & 2,440 & \\
\hline $\begin{array}{l}\text { Memorandum items: } \\
\text { (cumulative, in millions of U.S. dollars, unless otherwise indicated) }\end{array}$ & & & & & & \\
\hline $\begin{array}{l}\text { Net external program financing excluding any budget grants related to Internally Displaced Persons } \\
\text { (IDPs), program portion of Tokyo-related disbursements, and Fund financing to the budget } 4 /\end{array}$ & -166 & 737 & 95 & -928 & -1082 & \\
\hline Actual $5 /$ & & & -40 & -846 & $\begin{array}{l}-1,082 \\
-1,664\end{array}$ & 152 \\
\hline Use of IMF resources by the budget 4 / & $\ldots$ & $\ldots$ & 715 & 1,072 & 1,430 & 0 \\
\hline Actual & $\ldots$ & $\cdots$ & 745 & 1,117 & 1,117 & \\
\hline External project grants & 24 & 176 & 320 & 169 & 530 & 767 \\
\hline Actual $5 /$ & $\ldots$ & $\ldots$ & 21 & 92 & 220 & \\
\hline External project grants, excl. IDP grants and Tokyo related grants $3 /$ & $\ldots$ & $\ldots$ & 172 & 49 & 70 & 177 \\
\hline Actual $5 /$ & $\cdots$ & $\cdots$ & 21 & 41 & 84 & \\
\hline Total IDP grants (project and budget) $4 /$ & $\ldots$ & $\ldots$ & 220 & 110 & 310 & 52 \\
\hline Actual & $\ldots$ & $\ldots$ & 0 & 52 & 52 & \\
\hline Tokyo-related disbursements, excl. multilateral sources 4 / & $\ldots$ & $\ldots$ & 342 & 1,177 & 1,962 & 2,220 \\
\hline Actual & $\cdots$ & $\cdots$ & 0 & 520 & 642 & \\
\hline Tokyo-related disbursements from multilateral sources $4 /$ & $\ldots$ & $\ldots$ & 0 & 0 & 100 & 150 \\
\hline Actual & $\ldots$ & $\ldots$ & 0 & 0 & 0 & \\
\hline $\begin{array}{l}\text { Foreign Exchange cash reserve requirement (CRR, incl. special CRR) deposits in SBP (stock in } \\
\text { millions of U.S. dollars) }\end{array}$ & 832 & 728 & 779 & 808 & 845 & 875 \\
\hline Weekly cash reserve requirement ratios (in percentage points) & & & & & & \\
\hline Rupee deposits (less than one year maturity) & 9 & 5 & 5 & 5 & 5 & 5 \\
\hline Rupee deposits (more than one year maturity) & 0 & 0 & 0 & 0 & 0 & 0 \\
\hline Foreign currency deposits CRR & 5 & 5 & 5 & 5 & 5 & 5 \\
\hline Foreign currency deposits special CRR & 15 & 15 & 15 & 15 & 15 & 15 \\
\hline Increase in SDR allocation (stock in millions of U.S. dollars) & $\ldots$ & $\ldots$ & 1,281 & 1,283 & 1,283 & $\ldots$ \\
\hline \multicolumn{7}{|l|}{$\begin{array}{l}\text { Note: } \\
{ }^{*} \text { denotes performance criteria. }\end{array}$} \\
\hline \multicolumn{7}{|c|}{$\begin{array}{l}\text { 1/ Excludes IMF. } \\
2 / \text { In addition to the quantitative performance criteria for end-December, end-March, and end-June the relevant purchases will also be subject to the completion of reviews with the fourth } \\
\text { review to be completed no earlier than February } 27,2010 \text {, and the fifth review to be completed no earlier than May } 30,2010 \text {. }\end{array}$} \\
\hline \multicolumn{7}{|c|}{$\begin{array}{l}\text { 3/ All end-March } 2010 \text { figures showns as "Actual" and as "Adjusted target" are reported on a preliminary basis. } \\
\text { 4/ To be used in assessing respective adjustors for program targets. }\end{array}$} \\
\hline
\end{tabular}

\section{CInternational Monetary Fund. Not for Redistribution}


Table 2. Pakistan: Structural Conditionality, December 2008-July 2010

\begin{tabular}{|c|c|c|}
\hline & Target date & Status \\
\hline \multicolumn{3}{|l|}{ Prior Actions } \\
\hline Submission of Federal VAT act to parliament and consistent Provincial VAT acts to provincial assemblies. & & Met \\
\hline \multicolumn{3}{|l|}{ Structural Benchmarks } \\
\hline A contingency plan for handling problem private banks will be finalized. & end-December 2008 & Met \\
\hline $\begin{array}{l}\text { A full description of required reforms in the area of tax administration, including an } \\
\text { action plan for harmonizing the GST and income tax administration will be finalized. }\end{array}$ & end-December 2008 & Met \\
\hline $\begin{array}{l}\text { In close collaboration with the World Bank, the government will finalize the schedule } \\
\text { for further electricity tariff adjustments during 2008/09, with a view to eliminating } \\
\text { tariff differential subsidies by end-June } 2009 \text {. }\end{array}$ & end-December 2008 & Met $1 /$ \\
\hline The SBP's provision of foreign exchange for furnace oil will be eliminated. & February 1, 2009 & Met \\
\hline $\begin{array}{l}\text { In close collaboration with the World Bank, the government will develop a strategy } \\
\text { and a time-bound action plan for the adoption of specific measures to strengthen } \\
\text { the social safety net and improve targeting to the poor. }\end{array}$ & end-March, 2009 & Met \\
\hline The government will prepare a plan for eliminating the inter-corporate circular debt. & end-March, 2009 & Met with a delay \\
\hline The transition to a treasury single account will be completed. & end-June 2010 & Underway $2 /$ \\
\hline $\begin{array}{l}\text { Amendments to the banking legislation will be submitted to Parliament to enhance } \\
\text { the effectiveness of SBP enforcement powers in the area of banking supervision. }\end{array}$ & end-August 2009 & Met with a delay \\
\hline $\begin{array}{l}\text { 1. Government approval of regulations to (i) form new occupational groups within the FBR; } \\
\text { and (ii) revise the structures of Regional Taxpayer Offices and Large Taxpayer Units. } \\
\text { 2. Amendment of all relevant laws and rules. }\end{array}$ & September 15, 2009 & (i) Met; (ii) Met with a delay \\
\hline $\begin{array}{l}\text { The government will submit draft legislative amendments to parliament to harmonize } \\
\text { the income tax and GST laws, including for tax administration purposes. }\end{array}$ & end-September 2009 & Met with a delay $3 /$ \\
\hline Submission of the VAT law to Parliament. & end-December 2009 & Met with a delay 4 / \\
\hline $\begin{array}{l}\text { An expedited sales tax refund system will be implemented in all Regional Taxpayer Offices and Large } \\
\text { Taxpayer Units to ensure direct input of refund requests and prompt processing and confirmation of } \\
\text { refunds. }\end{array}$ & end-March 2010 & Underway $5 /$ \\
\hline $\begin{array}{l}\text { An independent review of the Pakistan Improvement of Financial Reporting and Auditing Project (PIFRA), } \\
\text { to improve reporting, lay the basis for commitment control, and assess in which institution PIFRA should be } \\
\text { located. }\end{array}$ & end-June 2010 & \\
\hline A full implementation of the VAT. & July 1, 2010 & \\
\hline
\end{tabular}

1/ The original plan was superseded by a plan that was agreed upon on July 15, 2009 that envisages elimination of tariff differential subsidies by August 2010

2/ In October 2008, the authorities took steps to prevent accumulation of unspent balances in accounts outside the Federal Consolidated Fund and made the use of assignment accounts by ministries under the Account No.1 fully operational for budget expenditures.

MoF has collected information about balances held by federal government entities in commercial bank accounts.

The target date has been revised from end June-2009.

$3 /$ An ordinance was issued on October 28. It will need to be augmented and legislated at a later stage to be made consistent with the new VAT law

that is being prepared.

4/ The federal part of the VAT law package was submitted to the parliament on February 25, 2010. The provincial laws were submitted to provincial assemblies by March 27.

5/ On March 29, the FBR issued a notification allowing the expeditious processing and payment of refunds to manufacturing exporters registered at the Lahore Regional Taxpayers Office from tax period April, 2010 and to all manufacturing exporters registered at other RTOs and LTUs from tax period July, 2010.

\section{CInternational Monetary Fund. Not for Redistribution}




\section{Attachment III. Pakistan: Technical Memorandum of Understanding on the PROGRAM SUPPORTED UNDER THE STAND-BY ARRANGEMENT}

May 3, 2010

1. This Technical Memorandum of Understanding (TMU) describes the monitoring arrangements under the SBA-supported program. It replaces from April 1, 2010 onwards the TMU dated December 11, 2009. Throughout, unless otherwise stated, "government" is meant to comprise the federal and provincial governments.

\section{DeFinitions OF MONITORING VARIABLES}

\section{Valuation of foreign exchange denominated assets, liabilities, and foreign} exchange flows. For the purposes of program monitoring under the program, all assets and liabilities as well as debt contracted, denominated in SDRs or in currencies other than the U.S. dollar, will be converted into U.S. dollars at the exchange rates prevailing at test dates, as posted by the State Bank of Pakistan (SBP) on its web site. Net external budget financing and external cash grants will be converted into Pakistani rupees at the exchange rates prevailing at the day of the transaction, as posted by the SBP on its web site, unless otherwise indicated.

3. Reserve money (RM) is defined as the sum of: currency outside scheduled banks (deposit money banks); scheduled banks' domestic cash in vaults; scheduled banks' required and excess rupee deposits with the SBP; scheduled banks' foreign exchange deposits with the SBP; and deposits of the rest of the economy with the SBP, excluding those held by the federal and provincial governments and the SBP staff retirement accounts.

4. Net foreign assets (NFA) of the SBP are defined as the difference between its foreign assets and foreign liabilities. Foreign assets of the SBP consist of gold, foreign exchange, balances held outside Pakistan, foreign securities, foreign bills purchased and discounted, the reserve position with the IMF, and SDR holdings. The definition of foreign assets of the SBP will be consistent with the IMF Data Template on International Reserves and Foreign Currency Liquidity. Gold will be valued at $\$ 20.27$ per fine troy ounce. Foreign liabilities of the SBP include outstanding IMF credits (excluding net IMF financing to the budget as defined in paragraph 10 below), deposits with the SBP of foreign governments, foreign central banks, foreign deposit money banks, international organizations, and foreign nonbank financial institutions (NBFI).

5. Net domestic assets (NDA) of the SBP are defined as the difference between the RM and the NFA of the SBP.

6. Net borrowing from the banking system by the government is defined as the difference between the banking system's claims, on a cash basis, on the federal, provincial, and local governments and the deposits of the federal, provincial, and local governments with the banking system, including district government funds balances. For the purposes of this memorandum, claims on government exclude: credit for commodity 
operations; government deposits exclude outstanding balances in the Zakat Fund; and balances in the various privatization accounts kept by the government in the banking system. The stock of bonds which were issued to banks in substitution of outstanding nonperforming loans to certain public entities, and which are being fully serviced by the government, are included in banking system claims on government. Table 1 summarizes the calculations of net borrowing from the banking system by the government.

7. Net borrowing from the SBP by the government is defined as SBP claims on the government minus government deposits with the SBP. SBP claims on the government include government securities, treasury bills, ways and means advances, treasury currency, and debtor balances. SBP claims on the government exclude accrued profits on government securities. Government deposits with the SBP exclude the Zakat Fund and Privatization accounts (Table 1).

8. The definition of the overall budget deficit (excluding grants) under the program will be the consolidated budget deficit, excluding grants, and including the operations of district governments financed from local funds. It will be measured by the sum of (a) total net financing to the federal, provincial, and local governments; and (b) total external grants to the federal and provincial governments. The former is defined as the sum of (i) net external budget financing (see $\$ 11$ ); (ii) net borrowing from the banking system (as defined above); and (iii) net domestic nonbank financing (see $\mathbb{1 1 2}$ ). The total external grants are defined as the sum of project grants, cash external grants for budgetary support, capital grants reflecting the principal amounts of external debt cancellation or swaps, and other grants.

9. Net external program financing is defined to include external privatization receipts; budget support grants; budget support loans from multilateral (other than the IMF, but including World Bank and Asian Development Bank (ADB) budget support and structural adjustment loans), official bilateral budget support loans, and private sector sources (e.g., bonds); rescheduled government debt service and change in stock of external debt service arrears net of government debt amortization due on foreign loans, the latter including any accelerated amortization including related to debt swaps or debt cancellation recorded as capital grants. It also includes foreign loans onlent to financial institutions and companies (public or private) and emergency relief lending. Program financing excludes all external financing counted as reserve liabilities of the SBP (defined above). Amounts projected for net external program financing and external grants are provided in Table 2.

10. Net IMF financing to the budget is defined as the difference between Fund disbursements credited to the budget and repayments from the budget through the Ministry of Finance's dedicated account for Fund financing at the SBP.

11. Net external budget financing is defined as net external program financing minus privatization receipts, minus budget support grants, plus all other external loans for the financing of public projects or other federal or provincial budget expenditures, plus transfers of external privatization receipts from the privatization account to the budget, 
plus net IMF financing to the budget. Amounts projected for net external budget financing are provided in Table 2.

12. Net domestic nonbank financing of the budget is defined as follows: domestic privatization receipts transferred from the privatization accounts to the budget, plus the change during each reporting period, in the stock of (i) permanent debt, which consists of nonbank holdings of prize bonds, all federal bonds, and securities; plus (ii) floating debt held by nonbanks; plus (iii) unfunded debt, which consists of National Savings Scheme (NSS) debt, Postal Life Insurance, and the General Provident Fund (GPF); plus (iv) net deposits and reserves received by the government (public accounts deposits); plus (v) any other government borrowing from domestic nonbank sources net of repayments; minus (vi) government deposits with NBFIs. Nonbank holdings of permanent and floating debt is defined as total debt outstanding, as reported by the SBP, minus holdings of banks as per the monetary survey. Total T-bill and other relevant government debt are valued at discount value and exclude accrued interest.

\section{External debt ${ }^{1}$}

13. The performance criterion on contracting or guaranteeing of medium-term and long-term nonconcessional external debt by the government or the SBP applies not only to debt as defined in point No. 9 of the Guidelines on Performance Criteria with Respect to Foreign Debt (adopted by the IMF Executive Board on August 24, 2000), but also to commitments contracted or guaranteed for which value has not been received. Excluded from this performance criterion are (i) foreign currency deposit liabilities of the SBP; and (ii) the outstanding stock of debt of Foreign Exchange Bearer Certificates (FEBCs), Deposit Bearer Certificates (DBCs), and Foreign Currency Bearer Certificates (FCBCs). The performance criterion setting a limit on the outstanding stock of short-term external

\footnotetext{
${ }^{1}$ The definition of debt set forth in No. 9 of the guidelines reads as follows: "(a) For the purpose of this guideline, the term "debt" will be understood to mean a current, that is, not contingent, liability, created under a contractual arrangement through the provision of value in the form of assets (including currency) or services, and which requires the obligor to make one or more payments in the form of assets (including currency) or services, at some future point(s) in time; these payments will discharge the principal and/or interest liabilities incurred under the contract. Debts can take a number of forms, the primary ones being as follows: (i) loans, that is, advances of money to obligor by the lender made on the basis of an undertaking that the obligor will repay the funds in the future (including deposits, bonds, debentures, commercial loans, and buyers' credits) and temporary exchanges of assets that are equivalent to fully collateralized loans under which the obligor is required to repay the funds, and usually pay interest, by repurchasing the collateral from the buyer in the future (such as repurchase agreements and official swap arrangements); (ii) suppliers' credits, that is, contracts where the supplier permits the obligor to defer payments until some time after the date on which the goods are delivered or services are provided; and (iii) leases, that is, arrangements under which property is provided which the lessee has the right to use for one or more specified period(s) of time that are usually shorter than the total expected service life of the property, while the lessor retains the title to the property. For the purpose of the guideline, the debt is the present value (at the inception of the lease) of all lease payments expected to be made during the period of the agreement excluding those payments that cover the operation, repair or maintenance of the property. (b) Under the definition of debt set out in point 9 (a) above, arrears, penalties, and judicially awarded damages arising from the failure to make payment under a contractual obligation that constitutes debt are debt. Failure to make payment on an obligation that is not considered debt under this definition (for example, payment on delivery) will not give rise to debt."
} 
debt refers to debt (as defined in Footnote 1) with original maturity of up to and including one year. Medium- and long-term external debt comprises debt with initial maturity of over one year.

14. Nonconcessional borrowing is defined as borrowing with a grant element of less than 35 percent. $^{2}$ The discount rates used to calculate the grant element will be the sixmonth and ten-year Commercial Interest Reference Rates (CIRRs) averages, as computed by the Strategy and Policy Review Department of the IMF. Six-month CIRRs are updated mid-February and mid-August (covering the six-month period preceding the date of update) and the ten-year CIRRs averages are updated mid-December (covering a period of 10 years preceding the date of the update). Six-month CIRRs averages are to be used for loans whose maturity is less than 15 years, while ten-year CIRRs averages are to be used for loans whose maturity is equal or more than 15 years.

15. External payment arrears are defined as unpaid debt service by the government beyond the due date under the contract and any grace period.

16. Tokyo-related disbursements refers to disbursements of donor support (loans and grants) pledged at the donor conference held in Tokyo on April 17, 2009.

\section{AdJUSTORS}

\section{Adjustors related to net external program financing}

17. For paragraphs 18-21, the net external program financing excludes any budget grants related to Internally Displaced Persons (IDPs), the program portion of Tokyorelated disbursements, and Fund financing to the budget. They are projected as follows:

July 2009-June 2010: \$152 million

18. If the actual cumulative net external program financing in U.S. dollar terms is higher (lower) than projected, the difference between the actual cumulative net external financing and the projected amounts is defined as the excess (shortfall) in net external program financing in U.S. dollar terms. The excess (shortfall) in net external program financing in U.S. dollar terms multiplied by a fixed accounting exchange rate of Rs 83 per $\$ 1$ represents the excess (or shortfall) in net external program financing in rupee terms.

19. The ceiling on the consolidated overall budget deficit (excluding grants) will be adjusted downward by the shortfall in net external program financing in rupee terms by up to Rs 73.5 billion.

20. The floors on the NFA of the SBP will be adjusted upward (downward) by the excess (shortfall) in net external program financing in U.S. dollar terms as defined above.

\footnotetext{
${ }^{2}$ Following the methodology set out in "Limits on External Debt or Borrowing in Fund ArrangementsProposed Change in Implementation of the Revised Guidelines,” April 8, 1996.
} 
21. The ceilings on the NDA of the SBP will be adjusted downward (upward) by the excess (shortfall) in net external program financing in rupee terms as defined above.

22. The ceiling on net borrowing from the SBP by the government will be adjusted downward by the excess in net external program financing in rupee terms as defined above.

Adjustors related to Tokyo-related disbursements (excluding multilateral sources)

23. Tokyo-related disbursements (excluding multilateral sources) are projected as follows:

July 2009-June 2010: \$2,220 million

24. If the actual cumulative gross Tokyo-related disbursements (excluding multilateral sources) are lower than the projected amount, the difference between their projected and actual values in U.S. dollar terms is defined as the shortfall in Tokyo-related disbursements (excluding multilateral sources) in U.S. dollar terms. The shortfall in Tokyo-related disbursements (excluding multilateral sources) in U.S. dollar terms multiplied by a fixed accounting exchange rate of Rs 83 per $\$ 1$ represents the shortfall in Tokyo-related disbursements (excluding multilateral sources) in rupee terms. In such a case:

a. The ceiling on the consolidated overall budget deficit (excluding grants) will be adjusted downward by up Rs 58.97 billion (equivalent to $\$ 0.71$ billion valued at Rs 83 per \$1) for the shortfall in Tokyo-related disbursements (excluding multilateral sources) in rupee terms in excess of Rs 117.94 billion.

b. The floor on the NFA of the SBP will be adjusted downward by the amount equivalent to the shortfall in Tokyo-related disbursements (excluding multilateral sources) in U.S. dollar terms.

c. The ceiling on the NDA of the SBP will be adjusted upward by the amount equivalent to the shortfall in Tokyo-related disbursements (excluding multilateral sources) in rupee terms.

Adjustors related to Tokyo-related disbursements from multilateral sources

25. Tokyo-related disbursements from multilateral sources are projected as follows:

July 2009-June 2010: \$150 million

26. If the actual cumulative gross Tokyo-related disbursements from multilateral sources are lower than the projected amount, the difference between their projected and actual values in U.S. dollar terms is defined as the shortfall in Tokyo-related disbursements from multilateral sources in U.S. dollar terms. The shortfall in related disbursements Tokyo-related disbursements from multilateral sources in U.S. dollar terms 
multiplied by a fixed accounting exchange rate of Rs 83 per $\$ 1$ represents the shortfall in Tokyo-related disbursements from multilateral sources in rupee terms. In such a case:

a. The ceiling on the consolidated overall budget deficit (excluding grants) will be adjusted downward by the shortfall in Tokyo-related disbursements from multilateral sources in rupee terms.

b. The floor on the NFA of the SBP will be adjusted downward by the amount equivalent to the shortfall in Tokyo-related disbursements from multilateral sources in U.S. dollar terms.

c. The ceiling on the NDA of the SBP will be adjusted upward by the amount equivalent to the shortfall in Tokyo-related disbursements from multilateral sources in rupee terms.

\section{Adjustors related to net IMF financing to the budget}

27. Net IMF financing to the budget is projected as follows:

July 2009-June 2010: \$0 million

28. If actual net Fund financing to the budget in U.S. dollar terms is different from the projected amount, the difference in net Fund financing to the budget in U.S. dollar terms is defined as the difference in actual net Fund financing converted into U.S. dollar by the actual SDR/U.S. dollar exchange rates and the projected amount. In such a case,

a. The floor on the NFA of the SBP will be adjusted by the difference in Fund financing to the budget in U.S. dollar terms (upward if the difference is positive, downward if the difference is negative).

b. The ceiling on the NDA of the SBP will be adjusted by subtracting the difference in Fund financing to the budget in U.S. dollar terms converted into rupees at a fixed accounting exchange rate of Rs 83 per \$1 (downward if the difference is positive, upward if the difference is negative).

Adjustor related to external project grants excluding IDP grants and Tokyo-related grants

29. If the amount of external project grants excluding IDP grants and Tokyo-related grants is lower than assumed under the program (see Table 2), the ceiling on the consolidated overall budget deficit (excluding grants) will be adjusted downward for the cumulative shortfall in these grants. This adjustor will not be applied in addition to any adjustment on account of external project grants to the consolidated overall budget deficit (excluding grants) that is made under paragraphs 19, 24a, and 26a. 


\section{Adjustor related to total IDP grants (project and budget)}

30. If the total amount of IDP grants (including project and budget grants) is lower than assumed under the program (see Table 2), the ceiling on the consolidated overall budget deficit (excluding grants) will be adjusted downward for the cumulative shortfall in these grants. This adjustor will not be applied in addition to any adjustment on account of IDP grants to the consolidated overall budget deficit (excluding grants) that is made under paragraphs $19,24 \mathrm{a}$, and $26 \mathrm{a}$.

\section{Adjustor related to changes in regulations on required reserves}

31. The ceilings on the NDA of the SBP will also be adjusted downward/upward by the amount of (i) banks' Pakistani rupee reserves freed/seized by any reduction/increase of the daily CRR relative to the baseline assumption; and (ii) any reduction/increase in the reservable deposit base that is related to definitional changes, as per the following formula: $\Delta \mathrm{NDA}=\Delta \mathrm{rB}_{0}+\mathrm{r}_{0} \Delta \mathrm{B}+\Delta \mathrm{r} \Delta \mathrm{B}$, where $\mathrm{r}_{0}$ denotes the reserve requirement ratio prior to any change; $\mathrm{B}_{0}$ denotes the level of the reservable deposits in the initial definition; $\Delta \mathrm{r}$ is the change in the reserve requirement ratio; and $\Delta \mathrm{B}$ denotes the change in the reservable deposits as a result of definitional changes. In case of significant liquidity and other financial sector pressures, the SBP will engage in consultations with the IMF staff in order to reach an understanding on the appropriate monetary policy response.

\section{Adjustor related to the SBP's net position under foreign exchange forwards and swaps}

32. An adjustor to the NFA target of the SBP will be implemented to reflect changes in the SBP's net position under foreign exchange forwards and swaps. Specifically, the NFA target of the SBP will be adjusted upward/downward by the amount of the increase/decrease in the net SBP's position under foreign exchange forwards and swaps. The SBP's net exposure under foreign exchange forwards and swaps was $\$ 2.355$ billion at end-December 2009. The maximum SBP's net exposure under foreign exchange forwards and swaps is capped at \$2.5 billion for end-June 2010.

\section{Adjustor related to foreign currency deposits of resident banks with the SBP}

33. An adjustor to the NFA target of the SBP will be implemented to reflect changes in foreign currency deposits of resident banks. Specifically, the NFA target of the SBP will be adjusted upward/downward by the difference between the stock of foreign currency deposits of resident banks with the SBP and projections (see SMEFP, Table 1: quantitative criteria). The stock of foreign currency deposits of resident banks with the SBP was $\$ 808$ million at end-December 2009.

\section{Program Reporting ReQuirements}

34. The following information, including any revisions to historical data, will be provided to the Middle East and Central Asia Department of the IMF through the office of the Resident Representative of the IMF in Pakistan, within the timeframe indicated: 
- Monthly provisional statements on federal tax and nontax revenue, within one month.

- Deposits into and withdrawals from the privatization accounts for each quarter, within one month. Withdrawals will be reported with the following breakdown (i) those which constitute budgetary use of privatization proceeds; (ii) those which constitute costs of privatization; and (iii) other (with explanation of the purpose of other withdrawals), as well as with the breakdown between domestic and external privatization receipts.

- Quarterly statements on budgetary capital receipts and disbursements, including repayments of bonds, recovery of loans from provinces and "others," within two months.

- $\quad$ Monthly (unreconciled) provisional data on federal expenditure and net lending (with separate data on disbursements and repayments), within one month.

- Quarterly statement on consolidated budgetary expenditure, with federal data approved by the Accountant General Pakistan Revenue (AGPR), within two months.

- Quarterly numbers on expenditure on social programs.

- Quarterly data on the stock of domestic government debt, broken down by instrument, within one month (Table 3).

- Quarterly data on WAPDA receivables within one month.

- $\quad$ Monthly data on Outstanding Audited Price Differential Claims.

- $\quad$ Monthly data on external budget financing, including (i) loan-by-loan program disbursements in U.S. dollar terms and rupee terms converted at exchange rates prevailing at the time of each transaction; (ii) cumulative amortization in U.S. dollar terms and rupee terms converted at exchange rates prevailing at the time of each transaction; and (iii) cumulative project loan disbursements in U.S. dollar terms and in rupees converted at exchange rates prevailing at the time of each transaction. Tokyo and IDP related disbursement should be indicated separately.

- $\quad$ Monthly data on Banks’ Budgetary Support (Table 1) within one month.

- $\quad$ The following monthly monetary data on a last-Saturday basis within two weeks:

(i) monetary survey;

(ii) accounts of the SBP;

(iii) consolidated accounts of the scheduled banks;

(iv) banks' lending to the government;

(v) detailed table on net foreign assets (both for the SBP and scheduled banks); and

(vi) detailed table of scheduled banks' reserves with the SBP. 
- $\quad$ The same tables as in the preceding item, but on an end-month and end-quarter basis (last business day), both at current and program exchange rates, within one month.

- $\quad$ The SBP Table on outstanding stock of foreign currency deposits, amended to include the classification of new FCA according to the residency of the holder.

- $\quad$ Daily data on exchange rates (interbank, retail market, and Telegraphic Transfers for SBP purchases in the retail market), SBP's sales and purchases in the foreign exchange markets, swaps and forward outright sales, within two business days.

- $\quad$ Monthly data on the outstanding stock of the SBP's forward foreign currency operations, including swaps and outright forward sales and purchases, within two weeks. The terms of any new transactions (including rollover/renewal of existing ones) will also be provided.

- $\quad$ Monthly data on the SBP's foreign exchange reserves, with details on the currencies, instruments, and institutions in which the reserves are held, within one month.

- Monthly data on SBP direct or bridge loans to nationalized banks in the context of the restructuring and privatization operation, within four weeks.

- $\quad$ Monthly data on any other quasi-fiscal operations undertaken by the SBP, on behalf of the government.

- Monthly data on SBP holding of discounted export finance credit under the export finance scheme, within one month.

- $\quad$ Monthly data on outstanding credit to agriculture under the Agriculture Mandatory Credit Targets, within one month.

- The following data on external debt, within one month:

(i) Quarterly stock of public- and publicly-guaranteed external debt (including deferred payments arrangements), by maturity (initial maturities of up to and including one year, and over one year), by creditor and by debtor (central government and publicly guaranteed);

(ii) Quarterly contracting or guaranteeing of nonconcessional medium- and long-term government debt; and

(iii) Information on any rescheduling on public- and publicly-guaranteed debt reached with creditors.

- $\quad$ Quarterly data on external payments arrears on public and publicly guaranteed debt with details as in (i) of the preceding item within one month. 
- Copies of new or revised ordinances/circulars regarding changes in tax policy, tax administration, foreign exchange market regulations, and banking regulations no later than three days after official issuance, or notification that ordinances have been posted on the Federal Board or Revenue (FBR) and SBP websites.

- $\quad$ Copies of official notification of changes in gas and electricity tariffs and any surcharges (automatic or structural) and in ex-refinery petroleum product prices as well as of gas and petroleum surcharges/levies.

- $\quad$ Monthly data on the import parity prices as well as central depot prices of the six major oil products, within one month.

- Quarterly data on KESC and WAPDA loans and debt outstanding, within one month.

- Upon the adoption of the plan for the elimination of inter-corporate circular debt, monthly reports on inter-corporate circular debt will be reported within one month.

- Information on new liabilities incurred or guaranteed by the government in settling the circular-debt, including government guarantees of Term Financing Certificates (TFCs) issued by Pakistan Electric Power Company (PEPCO). The information will include the size of the government exposure, the duration of the guarantee or claim, and any other provisions relevant for the government's exposure.

- Information on the transactions between the Ministry of Finance and the SBP with regard to the use of Fund resources for budget purposes on monthly basis. 
Table 1. Pakistan Budgetary Support

(In million Rupees)

\begin{tabular}{|c|c|c|c|c|c|}
\hline & & 30-Jun-09 & 30-Sep-09 & 31-Dec-09 & 31-Mar-10 \\
\hline \multirow[t]{20}{*}{ A. } & Central Government & $1,849,101$ & $1,867,809$ & $1,984,554$ & $2,112,513$ \\
\hline & Scheduled Banks & 737,490 & 861,515 & 936,705 & 957,606 \\
\hline & Government Securities & 214,164 & 225,290 & 234,149 & 243,041 \\
\hline & Treasury Bills & 756,955 & 890,690 & 982,955 & $1,018,764$ \\
\hline & Government Deposits & $-233,630$ & $-254,465$ & $-280,400$ & $-304,199$ \\
\hline & State Bank & $1,111,612$ & $1,006,294$ & $1,047,850$ & $1,154,906$ \\
\hline & Government Securities & 3,144 & 3,144 & 3,144 & 3,144 \\
\hline & Accrued Profit on MRTBs & 35,131 & 33,126 & 36,348 & 34,187 \\
\hline & Treasury Bills & $1,107,858$ & $1,010,757$ & $1,147,657$ & $1,174,669$ \\
\hline & MTFBs purchased for replenishment of cash balances & $1,098,349$ & $1,001,248$ & $1,138,148$ & $1,165,160$ \\
\hline & Adhoc Treasury Bills & 0 & 0 & 0 & 0 \\
\hline & Ways and Means Advances & 0 & 0 & 0 & 0 \\
\hline & Treasury Currency & 8,153 & 8,153 & 8,153 & 8,153 \\
\hline & Debtor Balances (Excl. Zakat Fund) & 0 & 0 & 0 & 0 \\
\hline & Government Deposits & $-48,137$ & $-54,348$ & $-152,915$ & $-70,710$ \\
\hline & (Excl. Zakat and Privatization Fund) & 0 & 0 & 0 & 0 \\
\hline & Special Account-Debt Repayment & 0 & 0 & & 0 \\
\hline & Payment to HBL on a/c of HC\&EB & -287 & -287 & -287 & -287 \\
\hline & Adjustment for use of Privatization Proceeds & & & & \\
\hline & for Debt Retirement & 5,749 & 5,749 & 5,749 & 5,749 \\
\hline \multirow[t]{12}{*}{ B. } & Provincial Governments & $-168,079$ & $-148,711$ & $-172,729$ & $-192,503$ \\
\hline & Scheduled Banks $(a+b-c)$ & $-221,114$ & $-222,448$ & $-226,892$ & $-231,297$ \\
\hline & Government Securities & 0 & 0 & & 0 \\
\hline & Advances to Punjab Government for Cooperatives & 1,024 & 1,024 & 1,024 & 1,024 \\
\hline & Government Deposits & $-222,138$ & $-223,472$ & $-227,916$ & $-232,321$ \\
\hline & State Bank & 53,035 & 73,737 & 54,163 & 38,794 \\
\hline & Debtor Balances (Excl. Zakat Fund) & 75,381 & 101,091 & 92,233 & 73,126 \\
\hline & Ways and Means Advances & 0 & 0 & 0 & 0 \\
\hline & Government Deposits (Excl.Zakat Fund) & $-22,346$ & $-27,354$ & $-38,070$ & $-34,332$ \\
\hline & Net Govt. Budgetary Borrowings from the Banking system & $1,681,022$ & $1,719,098$ & $1,811,825$ & $1,920,010$ \\
\hline & Through SBP & $1,164,647$ & $1,080,031$ & $1,102,013$ & $1,193,701$ \\
\hline & Through Scheduled Banks & 516,375 & 639,068 & 709,812 & 726,309 \\
\hline \multicolumn{6}{|c|}{ Memorandum Items } \\
\hline & Accrued Profit on SBP holding of MRTBs & 35,131 & 33,126 & 36,348 & 34,187 \\
\hline & Scheduled banks ' deposits of Privitization Commission & $-1,580$ & $-2,380$ & $-2,057$ & $-1,996$ \\
\hline & Outstanding amount of MTBs (Primary market; discounted value) & 739,475 & 871,341 & 942,567 & 971,767 \\
\hline \multicolumn{6}{|c|}{ Net Goverment Borrowings (Cash basis) } \\
\hline & From Banking System & $1,629,991$ & $1,669,004$ & $1,737,145$ & $1,840,822$ \\
\hline & From SBP & $1,129,516$ & $1,046,905$ & $1,065,664$ & $1,159,514$ \\
\hline & From Scheduled Banks & 500,475 & 622,098 & 671,481 & 681,308 \\
\hline
\end{tabular}

Source: State Bank of Pakistan

CInternational Monetary Fund. Not for Redistribution 
Table 2. Pakistan: External Financing For Budget for 2008/09 and 2009/10 (In millions of U.S. dollars)

\begin{tabular}{|c|c|c|c|c|c|c|c|c|c|c|}
\hline & $\begin{aligned} \text { Act. } \\
\text { Jul.-Sep. }\end{aligned}$ & $\begin{array}{r}\text { Act. } \\
\text { Oct.-Dec. } \\
\end{array}$ & $\begin{array}{r}\text { Act. } \\
\text { Jan.-Mar. } \\
2008 / 09\end{array}$ & $\begin{array}{r}\begin{array}{r}\text { Act. } \\
\text { Apr.-Jun. }\end{array} \\
\end{array}$ & $\begin{array}{r}\text { Act. } \\
\text { Total } \\
\end{array}$ & $\begin{array}{r}\text { Act. } \\
\text { Jul.-Sep. } \\
\end{array}$ & $\begin{array}{r}\text { Act. } \\
\text { Oct.-Dec. } \\
\end{array}$ & $\begin{array}{r}\begin{array}{r}\text { Proj. } \\
\text { Jan.-Mar. }\end{array} \\
2009 / 10\end{array}$ & $\begin{aligned} & \text { Proj. } \\
& \text { Apr.-Jun. }\end{aligned}$ & $\begin{array}{l}\text { Proj. } \\
\text { Total } \\
\end{array}$ \\
\hline Project Aid & 248 & 331 & 268 & 319 & 1,165 & 222 & 313 & 824 & 1,198 & 2,557 \\
\hline Grants, of which & 23 & 21 & 50 & 2 & 96 & 19 & 64 & 330 & 293 & 705 \\
\hline Regular & & & & & & 19 & 12 & 37 & 47 & 115 \\
\hline Tokyo pledge & 0 & 0 & 0 & 0 & 0 & 0 & 0 & 293 & 246 & 539 \\
\hline IDP grants & 0 & 0 & 0 & 0 & 0 & 0 & 52 & 0 & 0 & 52 \\
\hline Earth Quake grants & 1 & 2 & 3 & 74 & 80 & 2 & 7 & 23 & 29 & 62 \\
\hline World Bank, of which & 61 & 56 & 42 & 72 & 230 & 86 & 92 & 70 & 321 & 569 \\
\hline Regular & & & & & & 86 & 92 & 70 & 271 & 519 \\
\hline Tokyo pledge & 0 & 0 & 0 & 0 & 0 & 0 & 0 & 0 & 50 & 50 \\
\hline Earthquake & 7 & 21 & 0 & 20 & 49 & 0 & 0 & 0 & 0 & 0 \\
\hline ADB, of which & 111 & 85 & 53 & 75 & 324 & 76 & 102 & 52 & 100 & 331 \\
\hline Regular & & & & & & 76 & 102 & 52 & 100 & 331 \\
\hline Tokyo pledge & 0 & 0 & 0 & 0 & 0 & 0 & 0 & 0 & 0 & 0 \\
\hline Earthquake & 7 & 9 & 5 & 6 & 27 & 0 & 0 & 0 & 0 & 0 \\
\hline Other bilateral project loan, of which & 52 & 44 & 68 & 43 & 207 & 39 & 48 & 248 & 456 & 790 \\
\hline Regular & & & & & & 39 & 48 & 234 & 245 & 565 \\
\hline Tokyo pledge & 0 & 0 & 0 & 0 & 0 & 0 & 0 & 14 & 211 & 225 \\
\hline Other Earthquake Loans & 0 & 0 & 0 & 54 & 54 & 0 & 0 & 0 & 0 & 0 \\
\hline Commodity Aid & 0 & 123 & 52 & 0 & 175 & 0 & 0 & 100 & 0 & 100 \\
\hline Program Loans & 494 & 811 & 677 & 815 & 2,797 & 416 & 320 & 379 & 1,682 & 2,797 \\
\hline World Bank & 0 & 0 & 485 & 224 & 709 & 265 & 0 & 54 & 300 & 619 \\
\hline ADB & 494 & 100 & 96 & 492 & 1,182 & 152 & 0 & 0 & 1,070 & 1,222 \\
\hline Tokyo pledge, of which & 0 & 0 & 0 & 51 & 51 & 0 & 320 & 37 & 200 & 557 \\
\hline World Bank & 0 & 0 & 0 & 0 & 0 & 0 & 0 & 0 & 0 & 0 \\
\hline$A D B$ & 0 & 0 & 0 & 51 & 51 & 0 & 0 & 0 & 100 & 100 \\
\hline IDB (excl. Tokyo pledges) & 0 & 561 & 77 & 18 & 656 & 0 & 0 & 288 & 112 & 400 \\
\hline Short-term commercial & 0 & 150 & 19 & 31 & 200 & 0 & 0 & 0 & 0 & 0 \\
\hline Budgetary Grants & 40 & 30 & 56 & 5 & 131 & 0 & 60 & 52 & 5 & 117 \\
\hline Privatization & 0 & 0 & 0 & 0 & 0 & 0 & 0 & 0 & 800 & 800 \\
\hline GDRs & 0 & 0 & 0 & 0 & 0 & 0 & 0 & 0 & 0 & 0 \\
\hline Securitization/China & 0 & 0 & 500 & 0 & 500 & 0 & 0 & 0 & 0 & 0 \\
\hline IDP budget grants & 0 & 0 & 0 & 10 & 10 & 0 & 0 & 0 & 0 & 0 \\
\hline Saudi Arabia (Tokyo pledge) & $\ldots$ & $\ldots$ & $\ldots$ & $\ldots$ & $\ldots$ & 0 & 200 & 0 & 0 & 200 \\
\hline Other grants & $\ldots$ & $\ldots$ & $\ldots$ & $\ldots$ & $\ldots$ & 0 & 0 & 0 & 0 & 0 \\
\hline Budget financing from the Fund & $\ldots$ & $\ldots$ & & $\ldots$ & & 745 & 372 & 0 & -418 & 699 \\
\hline Amortization & 700 & 802 & 810 & 379 & 2,691 & 456 & 867 & 1,050 & 633 & 3,006 \\
\hline Medium and Long-term & 538 & 258 & 760 & 277 & 1,833 & 373 & 168 & 1,027 & 284 & 1,852 \\
\hline Euro bonds & 22 & 0 & 500 & 0 & 522 & 22 & 0 & 600 & 0 & 622 \\
\hline IDB>1 year & 200 & 0 & 0 & 0 & 200 & 0 & 0 & 0 & 0 & 0 \\
\hline Other & 316 & 258 & 260 & 277 & 1,111 & 351 & 168 & 427 & 284 & 1,230 \\
\hline Short-term & 162 & 544 & 50 & 102 & 858 & 83 & 698 & 23 & 349 & 1,153 \\
\hline IDB & 162 & 428 & 0 & 102 & 692 & 83 & 582 & 5 & 317 & 987 \\
\hline Commercial & 0 & 116 & 50 & 0 & 166 & 0 & 116 & 18 & 32 & 166 \\
\hline \multicolumn{11}{|l|}{$\begin{array}{l}\text { Memorandum items: } \\
\text { (cumulative from the beginning of fiscal year) }\end{array}$} \\
\hline Net budget financing & 82 & 452 & 1,142 & 1,903 & & 927 & 1,326 & 1,531 & 4,165 & 4,165 \\
\hline $\begin{array}{l}\text { Net program financing for the budget (excl. Tokyo related disbursements, IDP } \\
\text { grants, Fund financing to the budget) }\end{array}$ & -166 & -127 & 296 & 737 & & -40 & -846 & $-1,502$ & 152 & 152 \\
\hline Tokyo-related disbursements (excl. multilateral sources) $1 /$ & 0 & 0 & 0 & 0 & & 342 & 1,177 & 1,962 & 2,220 & 2,220 \\
\hline Tokyo-related disbursements from multilateral sources & 0 & 0 & 0 & 0 & & 0 & 0 & 0 & 150 & 150 \\
\hline Total project grants (cumulative) & & & & & & 21 & 92 & 446 & 767 & 767 \\
\hline External project grants, excl. IDP grants and Tokyo related grants & 24 & 47 & 100 & 176 & & 21 & 41 & 101 & 177 & 177 \\
\hline Total grants for IDP (budget and project) & 0 & 0 & 0 & 0 & & 0 & 52 & 52 & 52 & 52 \\
\hline
\end{tabular}

Sources: Pakistani authorities; and Fund staff projections.

$1 /$ To be used in assessing respective adjustors for program targets. 
Table 3. Pakistan: Total Outstanding Domestic Debt

(In billion Rupees)

\begin{tabular}{|c|c|c|c|}
\hline & Jun-08 & Jun-09 & Mar-10 \\
\hline A. Permanent Debt & 608.0 & 678.0 & 771.2 \\
\hline Market Loans & 3.0 & 2.9 & 2.9 \\
\hline Federal Government Bonds & 9.0 & 7.2 & 7.1 \\
\hline Income Tax Bonds & 0.0 & 0.0 & 0.0 \\
\hline Government Bonds (L.R.-1977) & 0.0 & 0.1 & 0.1 \\
\hline Special Government Bonds For SLIC (Capitalization) & 1.0 & 0.6 & 0.6 \\
\hline GOP ljara Sukuk 3 years & 0.0 & 27.8 & 42.2 \\
\hline $\begin{array}{l}\text { Government Bonds (issued to HBL for settlement of CBR } \\
\text { Refund) }\end{array}$ & 0.0 & 0.0 & 0.0 \\
\hline Bearer National Fund Bonds (BNFB) & 0.0 & 0.0 & 0.0 \\
\hline BNFB Roll Over-II & 0.0 & 0.0 & 0.0 \\
\hline Special National Fund Bonds & 0.0 & 0.0 & 0.0 \\
\hline Federal Investment Bonds (Auction) & 1.0 & 1.0 & 0.0 \\
\hline Federal Investment Bonds (TAP) & 0.0 & 0.0 & 0.0 \\
\hline Pakistan Investment Bonds (PIBs) & 412.0 & 441.0 & 493.4 \\
\hline Prize Bonds & 183.0 & 197.4 & 224.8 \\
\hline B. Floating Debt & $1,637.0$ & $1,904.0$ & $2,299.8$ \\
\hline Treasury Bills (3 Months) & 0.0 & 0.0 & 0.0 \\
\hline Market Treasury Bills (auction) & 537.0 & 796.1 & $1,106.7$ \\
\hline MTBs for Replenishment $1 /$ & $1,100.0$ & $1,107.9$ & $1,193.1$ \\
\hline C. Unfunded Debt & $1,020.0$ & $1,270.5$ & $1,411.7$ \\
\hline Defense Savings Certificates & 285.0 & 257.2 & 222.2 \\
\hline National Deposit Certificates & 0.0 & 0.0 & 0.0 \\
\hline Khas Deposit Certificates & 0.0 & 0.3 & 0.3 \\
\hline Special Savings Certificates (Registered) & 160.0 & 288.8 & 340.8 \\
\hline Special Savings Certificates (Bearer) & 0.0 & 0.3 & 0.3 \\
\hline Regular Income Certificates & 51.0 & 91.1 & 125.0 \\
\hline Premium Saving Certificates & 0.0 & 0.0 & 0.0 \\
\hline Bahbood Savings Certificates & 229.0 & 307.5 & 352.6 \\
\hline Khas Deposit Accounts & 0.0 & 0.3 & 0.3 \\
\hline National Deposit Accounts & 0.0 & 0.0 & 0.0 \\
\hline Savings Accounts & 28.0 & 16.8 & 15.6 \\
\hline Special Savings Accounts & 67.0 & 88.6 & 118.4 \\
\hline Mahana Amdani Accounts & 2.0 & 2.4 & 2.2 \\
\hline Pensioners' Benefit Accounts & 88.0 & 109.9 & 124.0 \\
\hline National Savings Bonds & 0.0 & 0.0 & 3.7 \\
\hline Postal Life Insurance & 67.0 & 67.1 & 67.1 \\
\hline GP Fund & 43.0 & 40.1 & 39.2 \\
\hline D. Total $(A+B+C)$ & $3,266.0$ & $3,852.6$ & $4,482.6$ \\
\hline
\end{tabular}

1/ Inclusive of outright sale of MTBs to commercial banks.

Source: State Bank of Pakistan. 


\section{INTERNATIONAL MONETARY FUND}

\section{PAKISTAN}

\section{Fourth Review Under the Stand-By Arrangement, Requests for Waivers of Performance Criteria, Modification of Performance Criteria, and Rephasing of Access \\ Informational Annex}

Prepared by Middle East and Central Asia Department

May 3, 2010

Contents

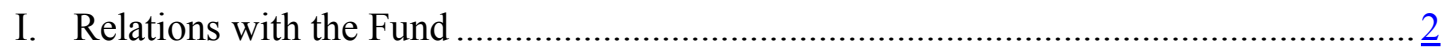

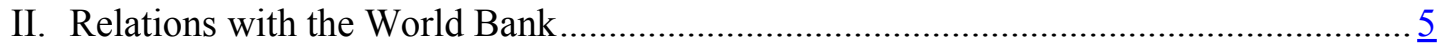




\section{ANNEX I. PAKISTAN: Relation WITH FUND}

As of March 31, 2010

I. Membership Status: Joined: 07/11/1950; Article VIII

II. General Resources Account:

Quota

Fund Holdings of Currency

Reserve position in Fund

III. SDR Department:

Net cumulative allocation

Holdings

IV. Outstanding Purchases and Loans:

Stand-by Arrangements

ECF arrangements $\underline{\text { SDR Million }}$

$1,033.70$

$5,202.92$

0.12

$\underline{\text { SDR Million }}$

988.56

865.94

$\underline{\text { SDR Million }}$

$4,169.34$

577.15
\% Quota

100.00

503.33

0.01

\% Allocation

100.00

87.60

\% Quota

403.34

55.83

\section{Latest Financial Arrangements:}

\begin{tabular}{|c|c|c|c|c|}
\hline Type & $\begin{array}{l}\text { Approval } \\
\text { Date }\end{array}$ & $\begin{array}{l}\text { Expiration } \\
\text { Date }\end{array}$ & $\begin{array}{l}\text { Amount Approved } \\
\text { (SDR Million) }\end{array}$ & $\begin{array}{l}\text { Amount Drawn } \\
\text { (SDR Million) }\end{array}$ \\
\hline Stand-By & $11 / 24 / 2008$ & $12 / 30 / 2010$ & $7,235.90$ & $4,169.34$ \\
\hline ECF & $12 / 06 / 2001$ & $12 / 05 / 2004$ & $1,033.70$ & 861.42 \\
\hline Stand-By & $11 / 29 / 2000$ & $09 / 30 / 2001$ & 465.00 & 465.00 \\
\hline
\end{tabular}

\section{Projected Payments to the Fund ${ }^{1,2}$}

(SDR Million; based on existing use of resources and present holdings of SDRs):

\begin{tabular}{lrrrrr} 
& \multicolumn{5}{c}{ Forthcoming } \\
\cline { 2 - 6 } & $\mathbf{2 0 1 0}$ & $\mathbf{2 0 1 1}$ & $\mathbf{2 0 1 2}$ & $\mathbf{2 0 1 3}$ & $\mathbf{2 0 1 4}$ \\
Principal & 137.83 & 172.28 & $1,418.11$ & $2,170.81$ & 847.45 \\
Charges/Interest & 55.34 & 73.85 & 62.89 & 28.37 & 6.77 \\
Total & 193.17 & 246.13 & $1,481.00$ & $2,199.17$ & 854.22
\end{tabular}

\section{Exchange System}

On May 19, 1999, the dual exchange system was unified, with all international transactions conducted at the interbank market exchange rate (FIBR). The Fund classifies Pakistan's exchange rate regime as floating. Pakistan has accepted the obligations of Article VIII, sections 2, 3, and 4. Pakistan is maintaining an exchange system free of restrictions on the making of payments and transfers for current international transactions

\footnotetext{
${ }^{1}$ This schedule presents all currently scheduled payments to the IMF, including repayment expectations and repayment obligations. The IMF Executive Board can extend repayment expectations (within predetermined limits) upon request by the member if its external payment position is not strong enough to meet the expectations without undue hardship or risk.

${ }^{2}$ When a member has overdue financial obligations outstanding for more than three months, the amount of such arrears will be shown in this section.
} 
following the elimination of the cash margin requirements on letters of credit in June 2009 and of restrictions on advance import payments against letters of credit in January 2010 .

\section{Last Article IV Consultation}

The last Article IV consultation (Country Report 09/123) was discussed by the Executive Board on March 23, 2009.

\section{Safeguards Assessments}

An update of the March 2009 safeguards assessment was completed in February 2010 in relation to the augmentation of access under the Stand-By Arrangement. The assessment found that: (i) efforts are continuing to strengthen the safeguards framework at the SBP, including improved transparency and a more proactive role by the Audit Committee; (ii) the SBP's legal framework continues to present a safeguards risk due to an unclear timetable for enacting amendments to the SBP Act, which address the autonomy of SBP and management of foreign reserves; and (iii) the treatment and use of Fund resources for budgetary support has been clarified in an agreement between the SBP and the Ministry of Finance. In their official response, the authorities agreed to the proposed timetable for implementation of most measures.

\section{FSAP Participation and ROSCs}

\begin{tabular}{|l|c|l|}
\hline Fiscal Transparency Module & $11 / 28 / 2000$ & (www.imf.org) \\
\hline Fiscal Transparency Module-Update & $11 / 22 / 2004$ & Country Report No. 04/416 \\
\hline $\begin{array}{l}\text { Financial System Stability Assessment } \\
\text { Financial Sector Assessment Program }\end{array}$ & $6 / 23 / 2004$ & Country Report No. 04/215 \\
\hline $\begin{array}{l}\text { Data Module and Detailed Assessment Using } \\
\text { Quality Assessment Framework }\end{array}$ & $11 / 29 / 2004$ & Country Report No. 04/398 \\
\hline $\begin{array}{l}\text { Data Module, Reassessment of Monetary } \\
\text { Statistics and Detailed Assessment Using } \\
\text { Quality Assessment Framework }\end{array}$ & $2 / 2 / 2007$ & Country Report No. 07/74 \\
\hline Fiscal Transparency Module-Draft Update & $2 / 13 / 2007$ & Country Report No. 08/129 \\
\hline $\begin{array}{l}\text { Financial System Stability Assessment, } \\
\text { Financial Sector Assessment Program Update }\end{array}$ & $9 / 22 / 2008$ & In progress \\
\hline
\end{tabular}

\section{Recent Technical Assistance}

\section{FAD}

January 2002: Fiscal data management, quality, and transparency. January 2003: Tax administration.

February/March 2003: Customs administration. April 2004: Fiscal reporting. April 2007: Public financial management. July and November 2009: Valued Added Tax law.

\section{MCM}

November/December 2004: Public debt reform and capacity building program (joint with World Bank). 
March/April 2005: Development of the Insurance Sector.

December 2006: Monetary policy framework.

April 2007: Monetary policy framework, the SBP's balance sheet, and the Banking

Services Corporation.

STA

February 2002: External sector statistics/SDDS subscription.

April/May 2005: National accounts and consumer price statistics.

May 2007: Statistics on the international investment position.

October 2009: Multisector statistics (remote technical assistance).

\section{LEG}

July 2008: Deposit Protection Fund.

July 2008: Central Bank Law.

August 2008: Banking Law.

\section{Resident Representative}

A resident representative has been stationed in Islamabad since August 1991. The current resident representative Mr. Paul Ross took up his post in October 2008. 


\title{
Annex II. Pakistan: Relations With the World Bank Group
}

\author{
Thomas Buckley, Senior Country Officer, Telephone: (202) 473-0075
}

1. Pakistan is among the largest recipients of World Bank financial assistance. The World Bank Group program in Pakistan consists of an integrated package of financial support, including IBRD lending, concessional IDA credits and IFC investments, along with complementary analytical and advisory services.

2. The Country Assistance Strategy (CAS) outlines The Bank's strategic approach to helping Pakistan achieve its development goals over a four year period. The Bank Group's Board of Directors endorsed a Country Assistance Strategy for Pakistan on June 1,2006 , covering fiscal years 2006 through 2009. The strategy addresses the key challenge of sustaining rapid growth in order to further reduce poverty.

3. The FY2006-2009 CAS was designed around three main pillars:

- Sustained Growth and Improved Competitiveness: The principal focus of this pillar is to support investments and reforms needed to improve the business environment for trade and investment and sustain rapid, private sector-led growth. Under this pillar, the Bank has provided support to key sectors such as agriculture and infrastructure, and helped the government strengthen macroeconomic management through improving public expenditures and supporting tax reforms.

- Strengthened Governance and Service Delivery: Priorities in this area include supporting further reforms and investment to increase efficiency, transparency, and accountability in the use of public resources, while supporting cross-cutting reforms needed to improve service delivery at all levels of government - with particular attention to health, education, water and sanitation, safety nets, and municipal services.

- Improved Lives and Protection of the Vulnerable: The Bank is focused on increasing investment in the education and health sectors to help build the skilled, healthy work force needed to sustain strong growth. This area of the CAS also featured targeted interventions to help the poor, including strengthening safety nets and targeted interventions and community-based approaches in rural areas.

4. The Bank is currently in the process of finalizing a new CAS which will cover fiscal years 2010 through 2014. The new CAS will be implemented in a period of uncertainty with a focus both on immediate needs dictated, in part, by ongoing conflict and the return to macroeconomic stability; but also a medium term approach that supports growth, service delivery, improved governance, human development and infrastructure investment. The objectives of the CAS will be aligned with Pakistani government's priorities as outlined in the Pakistan Poverty Reduction Strategy Paper II (PRSP-II) which was discussed by the Boards of the Bank and the IMF in March, 2009. The strategy will focus on restoring stability and maintaining growth, investing in human development resources and protecting the poor, as well as improving governance and service delivery.

5. IFC is an integral part of the World Bank Group's program in Pakistan. The IFC strategy in Pakistan, as expressed in the CAS, seeks to increase investments focusing on three main sectors: financial, SME and infrastructure. IFC has also initiated a substantial TA program in Pakistan to build capacity and address constraints of the SME, 
infrastructure and financial sectors. Looking ahead, IFC will address key constraints in Pakistan by mobilizing investments in power and infrastructure, and providing access to finance to MSMEs through financial intermediaries. Given the global economic situation IFC will support existing clients in the short-term with special focus on equity instruments and trade facilities with select banks. On the advisory front, IFC will provide technical assistance to build capacity of financial intermediaries, encourage public private partnerships in the infrastructure sector and supporting MSMEs.

IBRD/IDA financial operations since FY2004 are summarized below:

\section{Pakistan: World Bank Group Financial Operations}

(In millions of U.S. dollars)

\begin{tabular}{lrrrrrr}
\hline & FY2004 & FY2005 & FY2006 & FY2007 & FY2008 & FY2009 \\
\hline Commitments & & & & & & \\
$\quad$ IBRD & 50 & 347 & 315 & 100 & 174 & 0 \\
$\quad$ IDA & 731 & 500 & 1,161 & 726 & 296 & 1,609 \\
$\begin{array}{l}\text { Disbursements } \\
\quad \text { IBRD }\end{array}$ & 13 & 203 & 149 & 154 & 56 & 91 \\
$\quad$ IDA & 290 & 778 & 1,061 & 1030 & 267 & 848 \\
Repayments & & & & & & \\
$\quad$ IBRD & 277 & 299 & 289 & 265 & 270 & 253 \\
IDA & 96 & 111 & 117 & 170 & 143 & 224 \\
Debt disbursed and Outstanding & & & & & & \\
$\quad$ IBRD & 2,526 & 2,406 & 2,247 & 2,132 & 2,005 & 1,836 \\
IDA & 6,020 & 6,651 & 7,627 & 8,700 & 9,433 & 9,667 \\
\hline
\end{tabular}


Statement by the Staff Representative on Pakistan

Executive Board Meeting

May 14, 2010

1. This statement summarizes information that has become available since the issuance of the staff report (SM/10/76) on May 3, 2010. The new information does not change the thrust of the staff appraisal.

2. Final fiscal data show that the overall budget deficit (excluding grants) for July 2009-March 2010 was Rs 626 billion, Rs 10 billion lower than what was reported in Table 1 of the Supplementary Memorandum on Economic and Financial Policies of May 3,2010 . Accordingly, the margin of nonobservance (relative to the adjusted target) was Rs 52 billion or 0.3 percent of GDP. Other, minor, data revisions do not alter the qualitative assessment of performance (Table 1).

3. The current account deficit in July 2009-March 2010 was 1.4 percent of GDP (0.6 percent of GDP lower than projected in the staff report), compared with 5.1 percent of GDP in July 2008-March 2009. Official reserves increased to about $\$ 11.6$ billion in early May, following receipt of a further $\$ 656$ million from the U.S. Coalition Support Fund.

4. Inflation increased to 13.3 percent (y-o-y) in April 2010, reflecting both food and non-food price inflation, the latter due to higher petroleum product and cotton prices.

5. The Greek crisis has had a small impact. The Karachi stock market declined by 3.6 percent between April 22 and May 11, while Pakistan's EMBIG spread increased from 460 bps in late April to 543 bps on May 11.

6. The authorities continue to work toward the July 1 deadline for the introduction of VAT. Technical preparations are advancing. Some progress has been made in bridging the differences with the Province of Sindh, and the authorities continue to expect that the federal and all the provincial VAT bills will be approved by end-May.

7. Discussions continue with the World Bank and the Asian Development Bank staffs on a framework for reducing tariff differential subsidies and increasing supply in the electricity sector. The authorities have implemented measures to reduce load shedding including a shorter work week and opening hours for shops, and asked industry to operate on weekends and to move their non-working days to during the week. They are also increasing gas supplies for electricity generation. However, no announcement of the 6percent increase in electricity tariffs, to be implemented retroactively from April 1, 2010, has been made. 
8. The government recently announced a minimum monthly salary of Rs 7,000 for laborers, promised to regularize the rights of all contractual workers, and proposed to consolidate labor laws to protect workers' rights. Since monthly wages in the public sector already exceed Rs 7,000, the enforcement of the new minimum wage policy is not expected to have significant consequences for the federal and provincial budgets. 
Table 1. Pakistan: Quantitative Performance Criteria, June 2009-June 2010

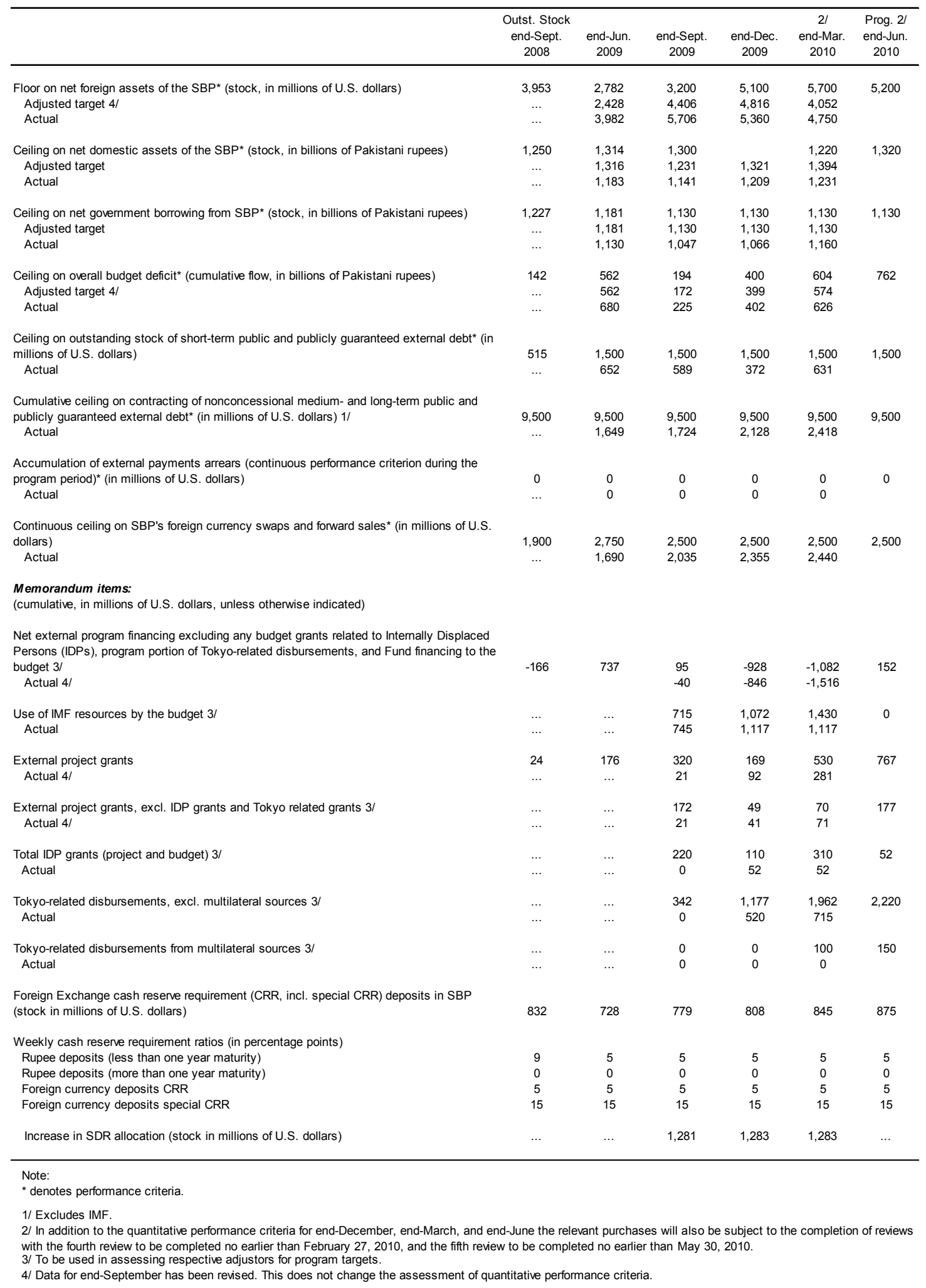

\section{CInternational Monetary Fund. Not for Redistribution}


May 14, 2010

\section{IMF Completes Fourth Review Under Stand-By Arrangement for Pakistan and Approves US\$1.13 billion Disbursement}

The Executive Board of the International Monetary Fund (IMF) today completed the fourth review of Pakistan's economic performance under a program supported by a Stand-By Arrangement (SBA). The completion of the review enables the immediate disbursement of an amount equivalent to SDR 766.7 million (about US\$1.13 billion), bringing total disbursements under the arrangement to an amount equivalent to SDR 4.94 billion (about US\$ 7.27 billion). The Board also approved rephasing three remaining disbursements into two, while keeping the total access under the arrangement unchanged.

The Executive Board also approved Pakistan's request for waivers for the non observance of two end-March 2010 quantitative performance criteria. These waivers were granted for overruns on the overall budget deficit and net government borrowing limits from the State Bank of Pakistan (SBP) on the grounds that their non observance was in part due to a temporary factor-the delay in the disbursements of foreign financing-and that adequate remedial actions have been agreed upon to address the remaining slippage.

Additionally, the Executive Board agreed to a request for modification of the end-June 2010 performance criteria for the budget deficit to increase the cumulative end-quarter ceilings by 0.15 percent of gross domestic product (GDP) to allow space for urgent security outlays and avoid undue cuts in other priority spending, and to raise the floor for the net foreign assets of the SBP by US $\$ 300$ million given a strengthened external position.

The 23-month SBA in an amount equivalent to SDR 5.1685 billion (about US\$7.61 billion) was approved on November 24, 2008 (see Press Release No. 08/303). On August 7, 2009, the SBA was augmented to an amount equivalent to SDR 7.2359 billion (about US\$10.66 billion) and extended to end 2010 (see Press Release No. 09/281).

Following the Executive Board's discussion on Pakistan, Mr. Murilo Portugal, Deputy Managing Director and Acting Chair, stated:

"Against a background of adverse security developments and a rapidly changing political environment, economic conditions have improved. Real GDP growth has begun to pick up and the external position has strengthened. Preparations for important and politically difficult tax reforms have moved forward, and there has been steady progress in financial sector reform. 
"Nevertheless, Pakistan's vulnerabilities remain high, due to persistent inflation, securityrelated spending pressures, energy-sector problems, and shortfalls in revenue collection and external financing. These challenges highlight the importance of pursuing a credible fiscal consolidation, maintaining a flexible exchange rate and a cautious stance to monetary policy, and improving governance. The authorities' resolve to press ahead with the structural reform agenda will also be key.

"The authorities reaffirmed their commitment to proceed with legal and administrative steps to ensure that the VAT is introduced on July 1 as scheduled, providing the needed tax revenue for investments in human resources, infrastructure, and poverty reduction. Its success depends crucially on prompt passage of consistent VAT laws by parliament and provincial assemblies, harmonization of other tax laws, and an effective refund system.

"Achieving the 2009/10 fiscal target will require strong efforts, including from the political leadership. Resolute continuation of tax collection efforts, tax administration reform, and expenditure restraint, together with timely disbursement of the pledged foreign financing will be critical to facilitate fiscal management.

"The risks posed by quasi-fiscal operations need to be addressed through reforming the electricity sector, cutting back losses at public enterprises, and managing losses from wheat procurement in a transparent manner. Steps are being taken to ensure that commodity operations do not crowd out credit to the private sector.

"The authorities are determined to accelerate the nationwide rollout of the new targeting system for the social safety net, with a view to easing hardship in a period of high inflation and sluggish growth," Mr. Murilo said. 


\section{Statement by Mr. Mojarrad and Mr. Iqbal on Pakistan Executive Director and Advisor for Pakistan May 14, 2010}

We thank staff for their continued commitment to assist Pakistan in addressing the economic challenges, as reflected in the high quality of this report. We wish also to convey our authorities' appreciation to Executive Directors and management for their steadfast support to Pakistan's adjustment and reform program. The economy of Pakistan has started to turn the corner and is now better poised to respond to evolving pressures and vulnerabilities.

Notwithstanding serious security challenges, the associated uncertainty, and the contractionary impact of the global crisis, the economy has come a long way since the approval of the stand-by arrangement some eighteen months ago. The authorities' strong commitment to the ambitious Fund-supported stabilization program has played a central role in this evolution. They have courageously implemented some very difficult structural reforms and have tightened domestic demand. Performance at end-December 2009 was generally in line with the program, and all quantitative and continuous performance criteria through end-March have been met, except for minor deviations from the endMarch ceilings on budget deficit, and net government borrowing from the State Bank of Pakistan (SBP). These deviations were on account of somewhat less than expected revenue, higher than expected security-related expenditures because of intensified military operations in Waziristan, and shortfalls in external disbursements, including under the Tokyo Agreement. ${ }^{1}$ The authorities are requesting waivers for their nonobservance. Progress has continued on important structural reforms, with virtually all structural benchmarks being met, albeit with a few delays.

There are signs of economic growth picking up, with incipient recovery in both manufacturing and agricultural output. However, investment outlays are yet to recover and unemployment pressures remain strong. Although there has been some temporary uptick in inflation in recent months, the authorities are determined to maintain tight demand management to expeditiously return to the path of declining inflation as envisaged by the program. It is gratifying to note that the year-on-year core inflation declined in March 2010 to below 10 percent from a high of 18.5 percent in March 2009.

The external current account deficit is expected to decline further to about 3.8 percent of GDP in 2009/10 from 8.4 percent in 2007/08, as restrained monetary policy and a more flexible exchange rate policy have been maintained; the fiscal deficit, despite inevitable increases in security-related outlays, is estimated to be reduced by $2 \frac{1}{2} 2$ percent of GDP over the same period to about 5 percent, which helped reduce public debt. Even though there have been delays in the disbursement of bilateral donor commitments under the Tokyo Agreement and with sluggish global demand negatively impacting exports, foreign

\footnotetext{
${ }^{1}$ Final fiscal data show that the overall budget deficit (excluding grants) for July 2009-March 2010 was Rs 626 billion, Rs 10 billion lower than what was reported in Table 1 of the Supplementary Memorandum on Economic and Financial Policies of May 3, 2010. Accordingly, the margin of nonobservance (relative to the adjusted target) was Rs 52 billion or 0.3 percent of GDP, instead of 0.4 percent of GDP estimated in the staff report.
} 
reserves have recovered and are expected to reach the equivalent of over four months of imports by the end of this fiscal year.

The authorities are acutely aware that the economy is poised at a crucial juncture. While major steps have been taken to reverse the deterioration of the past two years, they are determined to address the remaining challenges by durably improving the fiscal position, accelerating structural reforms, and enhancing efficiency in resource allocation under prudent demand management. They are firmly of the view that, notwithstanding current security-related pressures, unless energy shortages and inflation are quickly brought under firm control, it would be difficult to ensure resumption of sustained growth with internal and external stability. Hence the authorities' firm commitment to their reform agenda and macroeconomic stability as ably summarized in the staff report.

In line with the revamped strategy, the SBP is determined to continue its tight monetary policy to bring core inflation in line with the planned downward path. The current monetary policy stance appears to have helped in restraining inflationary expectations as reflected in banks' inclination to purchase longer maturity T-bills in the recent auctions. In line with the flexible exchange rate policy, the targeted reduction in inflation is also expected to stanch any real effective appreciation of the rupee and, thus, protect competitiveness.

An Energy Summit was held in April 2010, under the chairmanship of the Prime Minister and attended by all stakeholders, as well as representatives of the World Bank and the Asian Development Bank, to address challenges in the energy sector. In the event, a number of steps have been taken to conserve electricity and ensure that installed production capacity is better utilized, thus, improving growth outlook and employment opportunities. The near-term objective remains to increase electricity supply by increasing production capacity. In this context, additional natural gas is being allocated to power plants, while plans are afoot to commission 10 new power plants. It is important to note that electricity tariff rates have been increased by 60 percent over the 15 months ending in January 2010. The planned further 6 percent adjustment in April had to be delayed temporarily in order to ease the excessive burden on the population. The authorities are cognizant of the need to adjust and will continue to work toward eliminating the remaining tariff differential subsidies by adjusting electricity tariff rates in consultation with the World Bank and the Asian Development Bank; a comprehensive framework will be established by June 2010 for addressing the underlying shortages and subsidies.

The authorities have also taken decisive steps to redress the underlying quasi-fiscal pressures related to the energy sector's circular debt, which has militated against improving supply. A major portion of this debt will be handled by deducting bills owed by governmental institutions from their accounts, and the remainder will be refinanced by issuing bonds. The Federal government has already released Rs. 20 billion out of its dues of Rs. 66 billion.

The adjustment program will continue to be underpinned by a comprehensive fiscal reform strategy aimed at reducing the budget deficit to a sustainable level in the shortest possible time. The intensified response to the security situation, which was a major factor in the small overrun of the budget deficit as of end-March, 2010, will continue to weigh heavily. It is for this reason, and to ensure that critical developmental outlays are retained, that the Government has sought a small upward adjustment in the deficit target for end- 
2009/10. With the accelerated disbursements of security-related receipts under the U.S. Coalition Support Fund (CSF) and expected higher privatization proceeds ( $\$ 800$ million from the UAE Itesalat), notwithstanding continued shortfalls in disbursements under the Tokyo Agreement, it would be possible to meet the authorities' revised budget target for the year while allowing accumulation of the targeted additional foreign reserves. The Government will also endeavor to discontinue use of ad-hoc transfers from the SBP to cover revenue shortfalls by improving its own liquidity management.

A cornerstone of the ongoing fiscal reforms is to restructure the budget so as to eliminate its deficit bias and improve efficiency. In addition to the development of the mediumterm budget framework that will target a sustainable reduction in the deficit while increasing developmental outlays, devolution to provinces, strengthened tax administration, and reduction of fiscal risks in the public enterprise sector (particularly, in electricity), a permanent increase in revenue generation capacity is the focal point of reform. Despite political costs, the authorities have shown determination in submitting bills in the national and provincial assemblies for the introduction of the VAT by July 1, 2010. It is expected that the VAT legislation will be approved by end-May. The introduction of VAT will help in the enlargement of the tax base and revenue mobilization which will, among other things, go a long way in increasing domestic savings. Technical preparations for the implementation of VAT are moving apace. In addition to meeting the prior action requirements for the Fourth Review, this important measure will likely help address the gridlock that has so far stymied fundamental fiscal reforms. Moreover, the reform of tax administration is proceeding as planned. The budget financing options are being diversified in order to reduce reliance on SBP financing.

Progress has also been made on structural reforms in the financial sector. On May 6, 2010, the Standing Committee of the Senate passed the bill for amending the Banking Companies Ordinance in order to increase SBP's capacity, among other things, to deal with problem banks, strengthen governance in public financial institutions, and foster bank compliance with capital adequacy requirements. The National Assembly-the lower house- has already passed the bill, which will be sent back to the Senate for consideration. In addition, a law to enhance SBP's operational independence is under consideration in the Assembly. In conjunction with reforms to the commodity operations, especially wheat procurement, and the resultant containment of credit for such operations, it would be possible to increase space for private sector credit. The SBP is also working on mechanisms for encouraging credit to the SME sector where considerable potential for growth and employment exists.

In conclusion, the authorities have shown their determination to pursue very difficult, but necessary, reforms to implement the program despite lower than promised external assistance. The nonobservance of the end-March quantitative performance criteria on the overall budget deficit and net government borrowing from the SBP were temporary and reflected, in large part, factors beyond the authorities' control, such as delays in the disbursement of foreign financing. Notwithstanding the continued security-related pressures, the authorities are firmly committed to achieving program objectives in the period ahead. They will, therefore, highly appreciate Executive Directors' support for the requested waivers, program modifications, and rephasing of access. 